\title{
The Casimir force between real materials: Experiment and theory
}

\author{
G. L. Klimchitskaya*
}

Institute for Theoretical Physics, Leipzig University, Postfach 100920,

Leipzig D-04009, Germany

and North-West Technical University, Millionnaya Street 5, St. Petersburg 191065, Russia

\author{
U. Mohideen ${ }^{\dagger}$ \\ Department of Physics and Astronomy, University of California, Riverside, California \\ 92521, USA
}

\author{
V. M. Mostepanenko \\ Institute for Theoretical Physics, Leipzig University, Postfach 100920, \\ Leipzig D-04009, Germany \\ and Noncommercial Partnership "Scientific Instruments," Tverskaya Street 11, Moscow \\ 103905, Russia
}

(Published 21 December 2009)

\begin{abstract}
The physical origin of the Casimir force is connected with the existence of zero-point and thermal fluctuations. The Casimir effect is very general and finds applications in various fields of physics. This review is limited to the rapid progress at the intersection of experiment and theory that has been achieved in the last few years. It includes a critical assessment of the proposed approaches to the resolution of the puzzles arising in the applications of the Lifshitz theory of the van der Waals and Casimir forces to real materials. All the primary experiments on the measurement of the Casimir force between macroscopic bodies and the Casimir-Polder force between an atom and a wall that have been performed in the last decade are reviewed, including the theory needed for their interpretation. The methodology for the comparison between experiment and theory in the force-distance measurements is presented. The experimental and theoretical results described here provide a deeper understanding of the phenomenon of dispersion forces in real materials and offer guidance for the application of the Lifshitz theory to the interpretation of the measurement results.
\end{abstract}

DOI: $10.1103 /$ RevModPhys.81.1827

PACS number(s): 12.20.Fv, 12.20.Ds, 78.20.Ci, 85.85.+j

\section{CONTENTS}

I. Introduction

A. Fluctuations and the physical origin of the van der Waals and Casimir forces

B. Material and geometric properties in the theory of dispersion forces

1829

C. Modern Casimir force experiments

1830

D. Applications of the Casimir effect from fundamental physics to nanotechnology

1832

E. Structure of the review

II. Lifshitz's Theory of the Thermal van der Waals and Casimir Forces

A. Casimir interaction between two planar plates

B. Approximations for nonplanar boundary surfaces

C. Casimir-Polder atom-plate interaction and Bose-Einstein condensation

D. Puzzles in the application of the Lifshitz theory to real materials

1. Real metals

1836 *galina.klimchitskaya@itp.uni-leipzig.de

†umar.mohideen@ucr.edu

†vladimir.mostepanenko@itp.uni-leipzig.de
2. Real dielectrics

1840

III. How to Compare Theory and Experiment

A. Modeling of the optical properties of real materials

1. Kramers-Kronig relations

2. Calculation for $\mathrm{Au}$

3. Problem of spatial dispersion

B. Corrections to the Casimir force due to the imperfect geometry of interacting bodies

1. Surface roughness

2. Finite size and thickness

C. Quantitative comparison between experiment and theory in force-distance measurements

1. Experimental errors and precision

2. Comparison of experiment with theory

IV. Casimir Experiments with Metallic Test Bodies

A. Measurements of the Casimir force using an atomic force microscope

B. Precise determination of the Casimir pressure using a micromachined oscillator

C. Other experiments on the Casimir force between metal bodies

1. Torsion pendulum experiment

2. Micromechanical devices actuated by the Casimir force

1842

1842

1842

1843

1844

1845

1845

1847

1848

1848

1849

3. The experiment using the parallel plate configuration 
4. The Casimir force between thin metallic films

5. Dynamic measurement using an atomic force microscope

6. Ambient Casimir force measurements

7. Related measurements

D. Future prospects to measure the thermal Casimir force

V. Casimir Force Between a Metallic Sphere and a Semiconductor Plate

A. Motivation for use of semiconductors

B. Optically modulated Casimir force

C. Doped semiconductors with different charge carrier densities

1. $p$-type silicon

2. n-type silicon

D. Silicon plate with rectangular trenches

E. Future prospects to measure the Casimir force with semiconductor surfaces

1. The dielectric-metal transition

2. Casimir forces between a sphere and a plate with patterned geometry

3. Pulsating Casimir force

VI. Experiments on the Casimir-Polder Force

A. Demonstration of the thermal Casimir-Polder force

1. The force in thermal equilibrium

2. The force out of thermal equilibrium

B. Future prospects to measure the Casimir-Polder force in quantum reflection

C. Casimir-Polder interaction of atoms with carbon nanotubes

VII. Lateral Casimir Force and Casimir Torques

A. Lateral Casimir force between corrugated surfaces

B. Demonstration of the lateral Casimir force

C. The Casimir torque

VIII. Conclusions and Outlook

Acknowledgments

References

\section{INTRODUCTION}

\section{A. Fluctuations and the physical origin of the van der Waals and Casimir forces}

Long-range forces that are different from gravity but act between electrically neutral atoms or between an atom and a macrobody or between two macrobodies have been discussed for centuries. However, only after the development of quantum mechanics and quantum field theory has the physical picture of these forces become clear and the first quantitative results obtained. The origin of both the van der Waals and Casimir forces is connected with the existence of quantum fluctuations. For a nonpolar atom the mean value of the operator of the dipole moment in the ground state is equal to zero. However, due to quantum fluctuations, the mean value of the square of the dipole moment is not equal to zero. This leads to the existence of what are referred to as dispersion forces (Mahanty and Ninham, 1976; Parse- gian, 2005), the generic name for both the van der Waals and Casimir forces.

For atomic separations between a few angstroms and a few nanometers (which are much smaller than the characteristic absorption wavelength) the retardation effects are negligible. In this separation region the dispersion force is usually called the van der Waals force. This is a nonrelativistic quantum phenomenon and its theory was pioneered by London (1930). In the separation region of the van der Waals interaction, a virtual photon emitted by one atom can be absorbed by another atom during the lifetime of this photon as determined by the Heisenberg uncertainty relation. At relatively large atomic separations, of order of or larger than the characteristic absorption wavelength, relativistic retardation effects play an important role. At such separations the dispersion forces are usually called Casimir-Polder (for atom-atom and atom-wall interactions) or Casimir (for interaction between two macroscopic bodies) forces. These are both relativistic and quantum-mechanical phenomena first described by Casimir and Polder (1948) and by Casimir (1948), respectively. For atomic separations $\left|\boldsymbol{r}_{2}-\boldsymbol{r}_{1}\right|$ of order of the characteristic absorption wavelength, the virtual photon emitted by one atom cannot reach the second one during its lifetime. However, the operators of the quantized electric field at the points $\boldsymbol{r}_{1}$ and $\boldsymbol{r}_{2}$ in the vacuum state are correlated such that

$$
\left\langle E_{k}\left(t, \boldsymbol{r}_{1,2}\right)\right\rangle=0 \quad \text { but }\left\langle E_{k}\left(t, \boldsymbol{r}_{1}\right) E_{j}\left(t, \boldsymbol{r}_{2}\right)\right\rangle \neq 0 .
$$

As a result, the atoms situated at points $\boldsymbol{r}_{1}$ and $\boldsymbol{r}_{2}$ are characterized by fluctuating dipole moments and these fluctuations are correspondingly correlated, leading to the Casimir-Polder and Casimir forces.

The theoretical approach to the atom-wall and wallwall interactions developed by Casimir and Polder used ideal metal walls at zero temperature. The finite Casimir energy per unit area of two infinitely large parallel ideal metal walls separated by a distance $a$ (and the respective pressure) was found as a difference between the energies of zero-point (vacuum) oscillations of the electromagnetic field in the presence and in the absence of walls as

$$
E_{0}(a)=-\frac{\pi^{2}}{720} \frac{\hbar c}{a^{3}}, \quad P_{0}(a)=-\frac{\pi^{2}}{240} \frac{\hbar c}{a^{4}} .
$$

Ideal metals are characterized by perfect reflectivity at all frequencies which means that the absorption wavelength is zero. Thus, the results (2) are universal and valid at any separation distance. They do not transform to the nonrelativistic London forces at short separations. Due to the difference in these early theoretical approaches to the description of the dispersion forces, the van der Waals and Casimir-Polder (Casimir) forces were thought of as two different kinds of force rather than two limiting cases of a single physical phenomenon, as they are presently understood.

A unified theory of both the van der Waals and Casimir forces between plane parallel material plates in thermal equilibrium separated by a vacuum gap was de- 
veloped by Lifshitz (1956). Lifshitz's theory describes dispersion forces between dissipative media as a physical phenomenon caused by the fluctuating electromagnetic field that is always present in both the interior and the exterior of any medium. According to the fluctuationdissipation theorem, there is a connection between the spectrum of fluctuations of the physical quantity in an equilibrium dissipative medium and the generalized susceptibilities of this medium which describe its reaction to an external influence. Using the fluctuationdissipation theorem, Lifshitz derived the general formulas for the free energy and force of the dispersion interaction. In the limit of dilute bodies these formulas describe the dispersion forces acting between atoms and molecules. In the framework of the Lifshitz theory material properties are represented by the frequencydependent dielectric permittivities and atomic properties by the dynamic atomic polarizabilities. In the limiting cases of small and large separation distances, in comparison with the characteristic absorption wavelength, the Lifshitz theory reproduces the results obtained by London and by Casimir and Polder, respectively. It also describes the transition region between the nonrelativistic and relativistic areas. In fact, the fluctuating electromagnetic field in the Lifshitz theory is some classical analog of vacuum (zero-point) oscillations in the field-theoretical approach developed by Casimir. van Kampen et al. (1968), Ninham et al. (1970), Gerlach (1971), and Schram (1973) narrowed the distinction between the Casimir and Lifshitz approaches. They obtained the Lifshitz formulas for the free energy and force between two nondissipative material plates as the difference between the free energies of zero-point and thermal oscillations in the presence and in the absence of plates. The eigenfrequencies of these oscillations were found from the standard continuity boundary conditions for the electric field and magnetic induction on the surfaces of the dielectric plates. Later Barash and Ginzburg (1975) generalized this approach for the case of plates made of dissipative materials in thermal equilibrium with a heat reservoir. This generalization is presented by Milonni (1994) and by Mostepanenko and Trunov (1997). The applicability of the Lifshitz formula to dissipative materials was also demonstrated using the scattering approach (Genet, Lambrecht, and Reynaud, 2003). The assumption of a thermal equilibrium is basic for the Lifshitz theory. We repeatedly discuss the role of this assumption below.

Thus, the theoretical foundations of the Casimir interaction are based on two approaches. In one case the theory of the Casimir effect is based on the theory of equilibrium electromagnetic fluctuations in the media. In the second case the Casimir effect is a vacuum quantum effect resulting from the influence of external conditions and described by quantum field theory. In this case, boundary conditions are imposed (in place of material boundaries) which restrict the quantization volume and affect the spectrum of zero-point and thermal oscillations. In fact the two different approaches can be reconciled. There are derivations of the Lifshitz formu- las and more general results in different media where the dispersion force is viewed as a vacuum quantum effect (Tomaš, 2002; Raabe and Welsch, 2006). The common roots in the theory of electromagnetic oscillations relate the Casimir effect to other fluctuation phenomena, such as the radiative heat transfer through a vacuum gap (Volokitin and Persson, 2007). However, its origin in quantum field theory relates the Casimir effect to other quantum vacuum effects like the Lamb shift and the anomalous magnetic moment of an electron, where virtual particles play an important role (Jaffe, 2005).

During the last few years, far-reaching generalizations of the Lifshitz formulas were obtained which express the Casimir energy and force between two separated bodies of arbitrary shape in terms of matrices of infinite dimensions. This is often referred to as the representation of the Casimir energy in terms of functional determinants (Emig et al., 2006; Kenneth and Klich, 2006, 2008) or in terms of scattering matrices (Bulgac et al., 2006; Lambrecht et al., 2006). Several applications of new representations related to the subject of this review are discussed in the respective sections.

\section{B. Material and geometric properties in the theory of dispersion forces}

Both Casimir and Lifshitz considered dispersion forces in the configuration of two closely spaced plane parallel plates. However, real bodies may possess quite different geometrical properties. The case of an isolated body is also of much interest for the physics of dispersion forces. One unique feature of the Casimir force that has attracted widespread attention is its exotic geometry dependence. For example, it was first realized that the Casimir force can be repulsive for an ideal metal spherical shell (Boyer, 1968). Another example of the geometric dependence was found in ideal metal rectangular boxes, where the repulsive or attractive nature of the force depends on the ratio of the size of the sides (Lukosz, 1971). Both these results led to an explosion in theoretical studies of the shape dependence of the $\mathrm{Ca}$ simir force with ideal boundaries extensively reviewed (Milonni, 1994; Mostepanenko and Trunov, 1997; Milton, 2001). This subject is, however, outside the scope of our review, which deals only with effects pertaining to real material bodies.

In addition to the shape dependence mentioned above, there are more staid but no less important properties of interest for experimental measurements of the Casimir force between real bodies. Chief among these are the effect of the surface roughness on Casimir force, the role of curvature in the most often used sphere-plate configuration, the finite-size effects of the bodies used in the measurement, and the lateral Casimir force from corrugated surfaces. The last in particular exhibits diffractionlike effects, which can be experimentally observed. All these aspects are covered in the review.

Another feature of real material bodies differentiating them from ideal metal plates at zero temperature, as 
considered by Casimir, concerns the account of realistic material properties and thermal effects. As mentioned in Sec. I.A, in the Lifshitz theory real material properties are taken into account through the frequencydependent dielectric permittivity $\varepsilon(\omega)$. In its turn $\varepsilon(\omega)$ is expressed in terms of the complex index of refraction as $\varepsilon(\omega)=n^{2}(\omega)$. Both quantities are measurable over wide frequency regions. The magnitude of $\varepsilon(\omega)$ depends on the nature and structure of the material, including its electrical and optical properties. In the Lifshitz theory of dispersion forces the free energy and other thermodynamic quantities are expressed as functionals of $\varepsilon\left(i \xi_{l}\right)$, where the Matsubara frequencies $\xi_{l}=2 \pi k_{B} T l / \hbar$ are defined for all $l=0,1,2, \ldots\left(k_{B}\right.$ is the Boltzmann constant and $T$ is the temperature). In the alternative but mathematically equivalent formulation, the free energy is represented as a functional of $\varepsilon(\omega)$ defined along the real frequency axis (see Sec. II.A). The Lifshitz theory does not make a distinction among the physical processes contributing to the value of the dielectric permittivity. However, the applicability conditions of this theory, in particular thermal equilibrium, must be followed.

As is clear from the above, we call materials real (real metals, real dielectrics, etc.) to underline that this review deals with physical surfaces rather than with idealized ones such as ideal metals or dielectrics with constant dielectric permittivity and perfect geometric shape.

In the last ten years significant progress has been made in the measurement of the Casimir and CasimirPolder forces. This has added substantial information to the application of the Lifshitz theory to real materials. Historically the Lifshitz theory was proposed for ideal dielectrics, i.e., for materials having zero conductivity at any temperature. At nonzero temperature dielectrics are characterized by a small but physically real dc conductivity. From the start, the first papers (Lifshitz, 1956; Dzyaloshinskii et al., 1961) neglected this conductivity in the Lifshitz theory, and at zero frequency dielectric materials were characterized by a finite static dielectric permittivity $\varepsilon_{0}=\varepsilon(0)$. If, however, the dc conductivity of dielectrics at $T \neq 0$ is included, $\varepsilon(\omega)$ goes to infinity in the limit of zero frequency (see Sec. II.D). Geyer et al. (2005b) showed that the inclusion of even a negligibly small dc conductivity for the configuration of two parallel dielectric plates leads to an enormously large thermal correction to the Casimir free energy and pressure. If a physical effect is negligibly small, its inclusion in a general theory must not significantly influence the results. Thus, this thermal correction is surprising and it suggests that perhaps something is being done incorrectly. One might think that the dc conductivity of dielectric materials is not a minor effect that can be neglected in the theory of dispersion forces and that the discovered large thermal correction is physically real but was overlooked by the founders of the Lifshitz theory. However, as shown by Geyer et al. (2005b), the inclusion of the dc conductivity in the model of the dielectric response violates the Nernst heat theorem and thus makes the Lif- shitz theory inconsistent with thermodynamics. Moreover, a large thermal correction resulting from the simple inclusion of the dc conductivity was excluded experimentally in the experiments by Chen et al. (2007a, 2007b) and Obrecht et al. (2007) (see Secs. I.C, V.B, and VI.A). Below we illustrate by many examples that the dc conductivity of dielectric materials is unrelated to dispersion forces and its inclusion contradicts the applicability conditions of the Lifshitz theory.

For the Casimir force between real metals the situation is closely analogous to that of the dielectrics. Boström and Sernelius (2000) applied the Lifshitz theory to describe the free energy and force in the configuration of two parallel metal plates at a temperature $T$. The dielectric permittivity of the metal was obtained using the tabulated optical data for the complex index of refraction extrapolated to zero frequency by means of the Drude model (see Sec. III.A). This takes into account both the drift current of the conduction electrons including relaxation inherent to it and displacement currents connected with plasma oscillations of free electrons and interband transitions of core electrons. As a result, an enormously large thermal correction to the Casimir free energy and force was obtained from the Lifshitz theory at short separations below $1 \mu \mathrm{m}$, in qualitative disagreement with the case of ideal metal plates. The Casimir force between plates made of ideal metals at nonzero temperature was first treated independently by Brown and Maclay (1969) using thermal quantum field theory. Schwinger et al. (1978) demonstrated that the Lifshitz theory is in agreement with the case of ideal metals if the limit $\varepsilon(i \xi) \rightarrow \infty$ is taken first before the limit $\xi \rightarrow 0$. Bezerra et al. (2002b, 2004) showed that the inclusion of relaxation processes of conduction electrons in the Lifshitz theory violates the Nernst heat theorem for metals with perfect crystal lattices. This is also in violation of the classical limit at large separations (Feinberg et al., 2001; Scardicchio and Jaffe, 2006). The large thermal correction predicted by Boström and Sernelius (2000) was found to be inconsistent with the measurement data of the experiments by Lamoreaux (1997), by Decca, Fischbach, et al. (2003), by Decca, López, Fischbach, et al. (2005), Decca et al. (2007a, 2007b) (see Secs. I.C, IV.B, and IV.C.1). At the same time, the use of the free electron plasma model for the characterization of a metal (Dzyaloshinskii et al., 1961; Hargreaves, 1965; Schwinger et al., 1978; Mostepanenko and Trunov, 1985) leads to small thermal corrections at short separations in qualitative agreement with the case of ideal metal plates (Bordag et al., 2000b; Genet et al., 2000). The results obtained by neglecting the role of the conduction electrons connected with the drift current are in agreement with thermodynamics and with the classical limit (Bezerra et al., 2004). As argued below, the inclusion of a drift current is beyond the applicability conditions of the Lifshitz theory.

\section{Modern Casimir force experiments}

There were many measurements of the van der Waals and Casimir forces made before 1990, of which only the 
experiment by van Blockland and Overbeek (1978) with metallic surfaces can be considered as an unambiguous demonstration [a historical survey has been made by Bordag et al. (2001)]. The modern stage in Casimir experiments is characterized by the use of a new generation of precise laboratory techniques for the measurement of small forces and short separation distances, permitting one to determine the experimental precision and compare data with theory. The experiment by Lamoreaux (1997) using a torsion balance to measure the Casimir force between a gold-coated spherical lens and a plate was the first in this more recent series. The precision from $5 \%$ to $10 \%$ that might be achieved at separations of about $1 \mu \mathrm{m}$ (see Sec. IV.C.1 for more details) leads to the conclusion that the Drude model is an inadequate description in the theory of the thermal Casimir force.

A series of measurements with increased sensitivity of the Casimir force between a metallized sphere and a plate was performed using the atomic force microscope (Mohideen and Roy, 1998; Klimchitskaya et al., 1999; Roy et al., 1999; Harris et al., 2000; Chen, Klimchitskaya, Mohideen, and Mostepanenko, 2004). These experiments introduced the idea of using metallized polystyrene spheres which have very low mass, as one of the interacting surfaces. Even the first experiment in this series (Mohideen and Roy, 1998) demonstrated the role of the skin depth and the surface roughness corrections to the Casimir force at separations from 120 to $300 \mathrm{~nm}$. Note that the corrections to the skin depth are often referred to as the finite conductivity corrections. It was shown that the data are in disagreement with the ideal metal Casimir force whereas the inclusion of the finite conductivity and roughness corrections leads to excellent agreement between the data and theory (thermal corrections being negligibly small in this experiment). The experimental error in the measurement of the $\mathrm{Ca}$ simir force between $1 \%$ and $2 \%$ depending on the confidence level was justified at the shortest separations (Chen, Klimchitskaya, Mohideen, and Mostepanenko, 2004).

The gradient of the Casimir pressure between two parallel metallic plates was measured dynamically by Bressi et al. (2002). In this experiment small oscillations were induced on one of the plates at the resonant frequency and the frequency shift due to the Casimir force was measured. This frequency shift is proportional to the derivative of the Casimir force with respect to the separation distance between the plates (see Sec. IV.C.3).

Chen et al. (2002a, 2002b) measured the lateral Casimir force between a metallized sphere and a plate covered with longitudinal coaxial sinusoidal corrugations of equal periods (Sec. VII.B). This force, which was theoretically predicted by Golestanian and Kardar (1997, 1998), is a harmonic function of the phase shift between the corrugations on the sphere and the plate. The demonstrated phenomenon of the lateral Casimir force is promising for nanotechnology where, together with the normal Casimir force, it allows one to actuate any trans- lations of a device element entirely due to the presence of the zero-point vacuum oscillations.

The most precise measurements of the Casimir force between metallic surfaces were performed in a series of experiments by Decca, Fischbach, et al. (2003), by Decca, López, et al. (2003), by Decca et al. (2004, 2007a, 2007b), and by Decca, López, Fischbach, et al. (2005) using a micromechanical torsional oscillator. This new technique was first used in Casimir experiments by Chan et al. (2001a, 2001b) as a demonstration of the actuation of micromechanical and nanomechanical devices by the Casimir force (see Sec. IV.C.2). Although Decca et al. exploited the sphere-plate configuration, the application of the dynamic measurement mode and the proximity force approximation allowed them to determine the Casimir pressure between two parallel plates. In the last of this series of experiments (Decca et al., 2007a, 2007b) a $0.2 \%$ total experimental error was reported at a separation of $160 \mathrm{~nm}$. The systematic error was shown to dominate the random one in the total experimental error. This was achieved here for the first time in Casimir force measurements. The measurements of the Casimir pressure using the micromechanical torsional oscillator exclude the prediction of large thermal effects at a $99.9 \%$ confidence level for a wide separation region below $1 \mu \mathrm{m}$ (see Sec. IV.B).

The next series of recent Casimir experiments was devoted to the investigation of the Casimir interaction between a metallized sphere and a semiconductor plate (Chen et al., 2005, 2007a, 2007b; Chen, Klimchitskaya, et al., 2006; Chen, Mohideen, et al., 2006). In the early stages of this research it was demonstrated that a change of the charge carrier concentration in a semiconductor plate changes the value of the Casimir force. In the experiments by Chen et al. (2007a, 2007b) the Casimir force between the sphere and the plate was measured in the presence and in the absence of incident laser light on the plate. This allowed a fundamental test of the role of semiconductor conductivity properties in the Casimir force to be performed. The data were found to be in an excellent agreement with the Lifshitz theory if the dc conductivity of the semiconductor plate in the dark phase (no incident light) is disregarded. If, however, the dc conductivity of the plate in the dark phase is included, the theory was found to be inconsistent with data at a confidence level of $95 \%$ (see Sec. V.B).

Another important experiment was performed by Obrecht et al. (2007). This is the first measurement of the thermal Casimir-Polder force made both in thermal equilibrium and in the nonequilibrium case. The Casimir-Polder force between $\mathrm{Rb}$ atoms and a dielectric substrate changes the frequency of dipole oscillations excited in a Bose-Einstein condensate separated from a wall by a distance of a few micrometers. The fractional difference of this frequency calculated on the basis of the Lifshitz theory by disregarding the dc conductivity of the wall material was found to be in excellent agreement with data (Obrecht et al., 2007). Klimchitskaya and Mostepanenko (2008b) showed, however, that with inclusion of the small but physically real dc conductivity 
for the dielectric substrate the theory is inconsistent with data (see Sec. VI.A).

Geometry effects beyond the proximity force approximation were experimentally demonstrated by Chan et al. (2008) where the gradient of the Casimir force acting between a Au-coated sphere and Si plate with deep rectangular trenches was measured (Sec. V.D).

\section{Applications of the Casimir effect from fundamental physics to nanotechnology}

Both the van der Waals and Casimir forces find many applications spanning the range from the purely scientific to the mostly technological. The reason lies in the fluctuation nature of dispersion forces and the universal role played by fluctuations in different physical phenomena. Here we list only some of the main areas where the Casimir effect is important. For a detailed discussion of the applications of the Casimir effect, see Plunien et al. (1986), Mostepanenko and Trunov (1988), Krech (1994), Milonni (1994), Mostepanenko and Trunov (1997), Kardar and Golestanian (1999), Bordag et al. (2001), Milton (2001, 2004), Lamoreaux (2005), Parsegian (2005), Klimchitskaya and Mostepanenko (2006), and Milonni (2007).

A number of applications of the Casimir effect belong to the field of condensed matter physics (Krech, 1994) and nanotechnology (Buks and Roukes, 2001, 2002; Chumak et al., 2004). Areas of impact are multilayered structures, wetting phenomena, colloids, critical systems, adhesion of microelements in nanoelectromechanical systems, and absorption of different gases by nanostructures. In elementary particle physics the role of the $\mathrm{Ca}$ simir effect is very important. It is included in the calculation of hadron masses and provides an effective mechanism for the compactification of extra spatial dimensions in multidimensional physics (Mostepanenko and Trunov, 1997). In gravitation and cosmology the $\mathrm{Ca}-$ simir effect results in a nonzero vacuum energy in spaces with non-Euclidean topology, can drive the inflation process, and leads to interesting effects in brane models of the Universe (Saharian, 2006). The Casimir effect has been actively used for obtaining stronger constraints on the hypothetical long-range interactions predicted in many theoretical schemes beyond the standard model (Bordag et al., 1998, 1999, 2000a; Long et al., 1999; Fischbach et al., 2001; Mostepanenko and Novello, 2001; Decca, Fischbach, et al., 2003, Decca, López, Chan, et al., 2005, Decca, López, Fischbach, et al., 2005; Decca et al., 2007a, 2007b; Mostepanenko et al., 2008).

Additional physical phenomena where the Casimir effect is important are the quantum reflection of atoms on different surfaces and Bose-Einstein condensation. Casimir-Polder forces depend on real atomic and material properties and influence scattering amplitudes and condensation conditions (Antezza et al., 2004; Babb et al., 2004). Even in biophysics, a proper account of the Casimir force is required for understanding the interaction of biological membranes through a liquid layer.

The diverse applications of the Casimir effect in both fundamental and applied physics demand reliable theo- retical methods allowing the calculation of the Casimir force between real material bodies used in experiments. Below we demonstrate that the Lifshitz theory can be adapted to accomplish these ends.

\section{E. Structure of the review}

This review is devoted to the experimental and theoretical results obtained in the last ten years and related to the Casimir force between real materials. It is not intended to cover not less important but alternative results and methods related to the Casimir effect in ideal configurations and more complicated geometries. The latter will be touched on only if they provide important guidelines for the experimentally oriented theoretical approaches applicable to real materials.

In Sec. II we present the main results of the Lifshitz theory for the configurations of two planar plates and an atom near a plate. Some approximate methods applicable to nonplanar boundary surfaces are also considered. Section III discusses the problem of how to compare theory and experiment in the Casimir effect. Here we consider the modeling of the optical properties of real materials and corrections to the Casimir force due to surface roughness and the finite size of the interacting bodies. Special attention is paid to the quantitative measure of agreement between experiment and theory in the force-distance measurements. Section IV presents the main Casimir experiments with metallic test bodies, including those using an atomic force microscope and a micromechanical oscillator. The results obtained are compared with different theoretical computations using the Lifshitz theory. The prospects for measuring the thermal Casimir force between metals are also discussed. In Sec. V the measurements of the Casimir force between a metallic sphere and a semiconductor plate are presented. The discussion starts with the crucial experiment on the optically modulated Casimir force. The experiments with heavily doped semiconductors having different charge carrier densities are also discussed. Then we consider the recent experiment involving a semiconductor plate with deep rectangular trenches. The proposed experiments on the change in Casimir forces for a dielectric-metal phase transition, between a sphere and a plate with patterned geometry, and with the pulsating Casimir force are considered. Section VI is devoted to the measurements of the Casimir-Polder force. Here the experiment on the first measurement of the thermal Casimir-Polder force at large separations is presented. Section VII deals with the lateral Casimir force and Casimir torques. After an introduction to the subject, the first experiment on the measurement of the lateral Casimir force between corrugated surfaces is considered. This is followed by consideration of the Casimir torque between corrugated surfaces at small angles and between anisotropic surfaces. Section VIII contains our conclusions. 


\section{LIFSHITZ'S THEORY OF THE THERMAL VAN DER WAALS AND CASIMIR FORCES}

\section{A. Casimir interaction between two planar plates}

Consider two thick dissimilar plane parallel plates (semispaces) in thermal equilibrium at equal temperature $T$, separated by an empty gap of width $a$. The Lifshitz formula (Lifshitz, 1956; Dzyaloshinskii et al., 1961) represents the van der Waals and Casimir free energy and force per unit area (i.e., the pressure) in terms of the reflection coefficients $r_{\mathrm{TM}}^{(n)}\left(\omega, k_{\perp}\right)$ and $r_{\mathrm{TE}}^{(n)}\left(\omega, k_{\perp}\right)$ for the two independent polarizations of the electromagnetic field. Here $\omega$ is the frequency and $k_{\perp}$ is the magnitude of the projection of the wave vector onto the plane of the plates (the $z$ axis is perpendicular to the plates). The index $n=1,2$ labels the plates. Transverse magnetic (TM) polarization means that the magnetic field is perpendicular to the plane formed by $\boldsymbol{k}_{\perp}$ and the $z$ axis, while for transverse electric (TE) polarization the electric field is perpendicular to this plane. There are many different derivations of the Lifshitz formula in the literature based on different approaches: in the framework of quantum statistical physics, thermal quantum field theory in the Matsubara formulation, scattering theory, etc. [see, e.g., Schram (1973), Milonni (1994), Zhou and Spruch (1995), Bordag et al. (2001), and Genet, Lambrecht, and Reynaud (2003)]. In all the derivations the condition of thermal equilibrium is used. The final result is represented in one of two equivalent forms: as a summation over the Matsubara frequencies along the imaginary frequency axis or as an integral over real frequencies. The first representation is used more often as it is more convenient for computations. Here the Casimir free energy per unit area is given by

$$
\mathcal{F}(a, T)=\frac{k_{B} T}{2 \pi} \sum_{l=0}^{\infty} \Phi_{E}\left(\xi_{l}\right)
$$

where the prime on the summation sign means that the term for $l=0$ has to be multiplied by $1 / 2$ and

$$
\begin{aligned}
\Phi_{E}(x) \equiv & \int_{0}^{\infty} k_{\perp} d k_{\perp} \sum_{\alpha} \ln \left[1-r_{\alpha}^{(1)}\left(i x, k_{\perp}\right)\right. \\
& \left.\times r_{\alpha}^{(2)}\left(i x, k_{\perp}\right) e^{-2 a q}\right] .
\end{aligned}
$$

Here $\alpha$ denotes TM or TE, $\xi_{l}$ are the Matsubara frequencies, and

$$
q \equiv q\left(i x, k_{\perp}\right)=\sqrt{k_{\perp}^{2}+x^{2} / c^{2}} .
$$

Note that the function $\Phi_{E}$ in Eq. (3) depends on the real argument $x=\xi_{l}$. In the Lifshitz theory, the material media are described by the dielectric permittivity, depending only on the frequency (Lifshitz, 1956; Dzyaloshinskii et al., 1961; Lifshitz and Pitaevskii, 1980). The description of the dielectric properties by $\varepsilon(\omega)$ fully accounts for temporal dispersion but neglects the possible contributions of spatial dispersion to the van der Waals and Casimir forces. In the case of homogeneous nonmagnetic media, the reflection coefficients are

$$
r_{\mathrm{TM}}^{(n)}\left(i x, k_{\perp}\right)=\frac{\varepsilon^{(n)}(i x) q-k^{(n)}}{\varepsilon^{(n)}(i x) q+k^{(n)}}
$$

$$
r_{\mathrm{TE}}^{(n)}\left(i x, k_{\perp}\right)=\frac{q-k^{(n)}}{q+k^{(n)}}
$$

where

$$
k^{(n)} \equiv k^{(n)}\left(i x, k_{\perp}\right)=\sqrt{k_{\perp}^{2}+\varepsilon^{(n)}(i x) x^{2} / c^{2}} .
$$

We underline that the reflection coefficients (6) are the standard Fresnel reflection coefficients calculated, however, along the imaginary axis $i x$, where they are real. They are derived from the standard continuity conditions for the tangential and normal components of the electric field, magnetic induction, and electric displacement on the boundary planes, $E_{1 t}=E_{2 t}, B_{1 n}=B_{2 n}, D_{1 n}$ $=D_{2 n}$, and $B_{1 t}=B_{2 t}$ (where the subscripts $t$ and $n$ denote the tangential and normal components, respectively). Note that these conditions can be identically reformulated in Casimir spirit such that one deals with fields only external to the plates (Emig and Büscher, 2004).

The reflection properties of the electromagnetic waves on metal surfaces are often described in terms of the Leontovich surface impedance (Landau et al., 1984). The corresponding expressions for the reflection coefficients along the imaginary frequency axis can be found in Bezerra et al. (2001) and Geyer et al. (2003).

For plates of finite thickness or plates made of several layers, the reflection coefficients have a more complicated form depending on layer thicknesses [see, e.g., Zhou and Spruch (1995), Klimchitskaya et al. (2000), and Tomaš (2002)]. In the literature, one can find the reflection coefficients for planar multilayer magnetodielectrics with $\mu \neq 1$ (Buhmann et al., 2005; Tomaš, 2005). The reflection coefficients have a more complicated form if the plate material is anisotropic.

The Casimir pressure between two plates in thermal equilibrium at a temperature $T$ is determined from Eq. (3),

$$
P(a, T)=-\frac{\partial \mathcal{F}(a, T)}{\partial a}=-\frac{k_{B} T}{\pi} \sum_{l=0}^{\infty} \Phi_{P}\left(\xi_{l}\right),
$$

where

$$
\Phi_{P}(x) \equiv \int_{0}^{\infty} k_{\perp} d k_{\perp} q \sum_{\alpha}\left(\frac{e^{2 a q}}{r_{\alpha}^{(1)}\left(i x, k_{\perp}\right) r_{\alpha}^{(2)}\left(i x, k_{\perp}\right)}-1\right)^{-1}
$$

As mentioned, Eqs. (3) and (8) are useful for practical computations. For some purposes (for example, to investigate the comparative role of propagating and evanescent waves), the following form of the Casimir free energy expressed as integrals along the real frequency axis is convenient (Lifshitz, 1956; Bezerra et al., 2007): 


$$
\mathcal{F}(a, T)=\frac{\hbar}{4 \pi^{2}} \int_{0}^{\infty} d \omega \operatorname{coth} \frac{\hbar \omega}{2 k_{B} T} \operatorname{Im} \Phi_{E}(-i \omega) .
$$

Here the function $\Phi_{E}$ is defined by Eq. (4) with $x=-i \omega$, where $\omega$ is the real frequency. The propagating waves correspond to $k_{\perp}<\omega / c$, i.e., to a purely imaginary $q$. The evanescent waves correspond to $k_{\perp} \geqslant \omega / c$, i.e., to a real $q$. These problems, however, are beyond the scope of this review. They are considered, for instance, by Henkel et al. (2004), Intravaia and Lambrecht (2005), Bimonte (2006a, 2006b), and Intravaia et al. (2007).

Using the Abel-Plana formula (Mostepanenko and Trunov, 1997) or the Poisson summation formula (Mehra, 1967; Schwinger et al., 1978), one can present the free energy per unit area and the pressure in the following forms:

$$
\begin{gathered}
\mathcal{F}(a, T)=E(a)+\Delta \mathcal{F}(a, T), \\
P(a, T)=P(a)+\Delta P(a, T) .
\end{gathered}
$$

Here $E(a)$ is given by

$$
E(a)=\frac{\hbar}{4 \pi^{2}} \int_{0}^{\infty} d \xi \Phi_{E}(\xi) .
$$

Similarly, the quantity $P(a)$ in the second equality in Eq. (11) is

$$
P(a)=-\frac{\hbar}{2 \pi^{2}} \int_{0}^{\infty} d \xi \Phi_{P}(\xi)
$$

The second terms on the right-hand sides of equalities (11) are given by

$$
\begin{aligned}
& \Delta \mathcal{F}(a, T)=\frac{i k_{B} T}{2 \pi} \int_{0}^{\infty} d t \frac{\Phi_{E}\left(i \xi_{1} t\right)-\Phi_{E}\left(-i \xi_{1} t\right)}{e^{2 \pi t}-1}, \\
& \Delta P(a, T)=-\frac{i k_{B} T}{\pi} \int_{0}^{\infty} d t \frac{\Phi_{P}\left(i \xi_{1} t\right)-\Phi_{P}\left(-i \xi_{1} t\right)}{e^{2 \pi t}-1} .
\end{aligned}
$$

Note that the quantities $E(a)$ and $P(a)$ in Eqs. (12) and (13) are often referred to as the Casimir energy per unit area and pressure at zero temperature, and $\Delta \mathcal{F}(a, T)$ and $\Delta P(a, T)$ in Eq. (14) are referred to as the thermal corrections to them. This terminology is, however, correct only for plate materials with temperature-independent properties. In this case the Casimir free energy and pressure depend on the temperature only through the Matsubara frequencies and the thermal corrections defined as

$$
\begin{gathered}
\Delta_{T} \mathcal{F}(a, T) \equiv \mathcal{F}(a, T)-\mathcal{F}(a, 0), \\
\Delta_{T} P(a, T) \equiv P(a, T)-P(a, 0)
\end{gathered}
$$

are the same as $\Delta \mathcal{F}(a, T)$ and $\Delta P(a, T)$ in Eq. (11). In this case $E(a)=\mathcal{F}(a, 0)$ and $P(a)=P(a, 0)$. If, however, the properties of the medium (for instance, the dielectric permittivity) depend on the temperature, then Eqs. (12)-(14) also contain a parametric dependence on the temperature. Thus, the quantities $E(a)$ and $P(a)$ are mixed quantities without a definite physical meaning (Bezerra et al., 2002b). They do not match $\mathcal{F}(a, T)$ and $P(a, 0)$, respectively. As a consequence, the thermal corrections $\Delta_{T} \mathcal{F}(a, T)$ and $\Delta_{T} P(a, T)$ do not coincide with $\Delta \mathcal{F}(a, T)$ and $\Delta P(a, T)$ in Eq. (14). Thus, the so-called zero-temperature Lifshitz formulas (12) and (13) can be used for the approximate calculation of the free energy and pressure only at rather small separation distances, where the computational results using Eqs. (3) and (12) practically coincide [Eqs. (8) and (13), respectively]. Usually this is the case for the calculation of the nonrelativistic van der Waals forces (Parsegian, 2005).

\section{B. Approximations for nonplanar boundary surfaces}

The Lifshitz theory was formulated for the case of two parallel plates. Experimentally it is hard to maintain two plates parallel at short separations. Because of this, most experiments (see Secs. IV, V, and VII) have been performed using the configuration of a sphere above a plate. The configuration of a cylinder above a plate also presents some advantages in comparison with the case of two parallel plates (see Sec. IV.D). Unfortunately, for many years it was not possible to obtain exact expressions for the Casimir force in these configurations. Thus, the approximate method of Derjaguin (1934), later called the proximity force approximation (PFA) (Blocki et al., 1977), was used to compare experiment with theory. According to this method, the Casimir energy in the gap between two smooth curved surfaces at short separation can be calculated approximately as a sum of energies between pairs of small parallel plates corresponding to the curved geometry of the gap. Specifically, under the condition $a \ll R$, where $R$ is the sphere or cylinder radius, the Casimir forces between the sphere or cylinder (per unit length) and a plate are given by

$$
F_{s}(a, T)=2 \pi R \mathcal{F}(a, T), \quad F_{c}(a, T)=\frac{15 \pi}{16} \sqrt{\frac{2 R}{a}} \mathcal{F}(a, T),
$$

respectively, where $\mathcal{F}(a, T)$ is the Casimir free energy per unit area in the configuration of two parallel plates, as defined in Eq. (3).

Within the PFA it is not possible to control the error of the approximation in Eq. (16). From dimensional considerations it is evident (Bordag et al., 2001) that the relative error in Eq. (16) should be of the order of $a / R$, but the numerical coefficient of this ratio is unknown. In fact, a rigorous determination of the error introduced by the application of the PFA requires a comparison of the approximate results in Eq. (16) with the exact analytic results or with some precise numerical computations in the respective configurations.

As discussed in Sec. I.B, during the last few years the finite representation of the Casimir energy for two separated bodies $A$ and $B$ in terms of the functional determinants was obtained. In this representation the Casimir energy can be written as (Kenneth and Klich, 2006, 2008) 


$$
\begin{aligned}
E(a) & =\frac{1}{2 \pi} \int_{0}^{\infty} d \xi \operatorname{Tr} \ln \left(1-\mathcal{T}^{A} \mathcal{G}_{\xi, A B}^{(0)} \mathcal{T}^{B} \mathcal{G}_{\xi, B A}^{(0)}\right) \\
& =\frac{1}{2 \pi} \int_{0}^{\infty} d \xi \ln \operatorname{det}\left(1-\mathcal{T}^{A} \mathcal{G}_{\xi, A B}^{(0)} \mathcal{T}^{B} \mathcal{G}_{\xi, B A}^{(0)}\right) .
\end{aligned}
$$

Here $\mathcal{G}_{\xi, A B}^{(0)}$ is the operator for the free space Green's function with the matrix elements $\left\langle\boldsymbol{r}\left|\mathcal{G}_{\xi, A B}^{(0)}\right| \boldsymbol{r}^{\prime}\right\rangle$, where $\boldsymbol{r}$ belongs to the body $A$ and $\boldsymbol{r}^{\prime}$ to $B . \mathcal{T}^{A}\left(\mathcal{T}^{B}\right)$ is the operator of the $T$ matrix for body $A$ (body $B$ ). The latter is widely used in light scattering theory, where it is the basic object for expressing the properties of the scatterers (Bohren and Huffmann, 2004). Using such representations, Emig et al. (2006) obtained the analytic results for the electromagnetic Casimir energy for an ideal metal cylinder above an ideal metal plane. Eventually, the result is expressed through the determinant of an infinite matrix with elements given in terms of the Bessel functions. The analytic asymptotic behavior of the exact Casimir energy at short separations was found by Bordag (2006). It results in the following expression for the Casimir force at $a \ll R$ :

$$
F_{c}(a, 0)=-\frac{\pi^{3}}{384 \sqrt{2}} \sqrt{\frac{R}{a}} \frac{\hbar c}{a^{3}}\left[1-\frac{1}{5}\left(\frac{20}{\pi^{2}}-\frac{7}{12}\right) \frac{a}{R}\right] .
$$

The PFA result in this case matches the first term on the right-hand side of Eq. (18). It can be obtained by replacing the free energy $\mathcal{F}(a, 0)$ in the second equality in Eq. (16) with the zero-temperature Casimir energy in the configuration of two ideal metal plates, $E_{0}(a)$ from Eq. (2).

Equation (18) is very important. It demonstrates that the relative error of the electromagnetic Casimir force between a cylinder and a plate calculated using the PFA is equal to $0.2886 a / R$. Thus, for typical experimental parameters of $R=100 \mu \mathrm{m}$ and $a=100 \mathrm{~nm}$, this error is approximately equal to only $0.03 \%$.

For a sphere above a plate made of ideal metals at $T=0$ PFA [Eq. (16)] leads to the result

$$
F_{s}(a, 0)=-\pi^{3} R \hbar c / 360 a^{3} .
$$

For this configuration the exact analytic solution in the electromagnetic case has not yet been obtained. The scalar Casimir energy for a sphere above a plate was found by Bordag (2006) and by Bulgac et al. (2006). The scalar Casimir energies for both a sphere and a cylinder above a plate have also been computed numerically using the wordline algorithms (Gies and Klingmüller, 2006a, 2006b), but it was noted that the Casimir energies for the Dirichlet scalar field should not be taken as an estimate for those in the electromagnetic case. Another numerical approach applicable in the electromagnetic case was developed by Rodrigues, Ibanescu, et al. (2007). This approach has not yet been applied at short separation distances of experimental interest. For an ideal metal sphere above an ideal metal plane a correction of order $a / R$ beyond the PFA was computed numerically by Emig (2008) for $a / R \geqslant 0.075$ and by Maia Neto et al.
(2008) for $a / R \geqslant 0.15$. In both cases the extrapolation of the obtained results to smaller $a / R$ leads to a coefficient near $a / R$ approximately equal to 1.4 .

In addition, the validity of the PFA for a sphere above a plate has been estimated experimentally (Krause $e t$ al., 2007) and the error introduced from the use of this approximation was shown to be less than $a / R$ (see Sec. IV.B for details). This is in disagreement with the extrapolations made by Emig (2008) and Maia Neto et al. (2008). To solve this contradiction, it is desirable to find the analytical form of the first correction beyond the PFA for a sphere above a plane, as in Eq. (18) for the cylinder-plane configuration.

\section{Casimir-Polder atom-plate interaction and Bose-Einstein condensation}

The interaction of atoms with a wall has long been investigated in different physical, chemical, and biological processes including absorption and scattering from various surfaces [see, e.g., Mahanty and Ninham (1976) and Israelashvili (1992)]. The general expression for the free energy of the atom-wall interaction can be obtained from Eq. (3) by considering one of the plates as a rarefied dielectric (Lifshitz, 1956; Lifshitz and Pitaevskii, 1980; Milonni, 1994). In doing so one expands the dielectric permittivity of a rarefied dielectric in powers of the number of atoms per unit volume $N$, keeping only the first-order contribution,

$$
\varepsilon(i \xi)=1+4 \pi \alpha(i \xi) N+O\left(N^{2}\right)
$$

Here $\alpha(\omega)$ is the dynamic polarizability of an atom. We consider only atoms in the ground (or metastable) state. The discussion of the interaction of excited atoms with a wall [see, e.g., Buhmann and Welsch (2007), and references therein] exceeds the scope of this review.

As a result, the free energy of atom-wall interaction with the wall temperature $T$ in thermal equilibrium can be presented in the form (Caride et al., 2005; Mostepanenko, Babb, et al., 2006)

$$
\mathcal{F}(a, T)=-k_{B} T \sum_{l=0}^{\infty} \alpha\left(i \xi_{l}\right) \int_{0}^{\infty} \frac{k_{\perp} d k_{\perp}}{q_{l}} \Phi_{A}\left(\xi_{l}, k_{\perp}\right),
$$

where

$$
\begin{aligned}
\Phi_{A}\left(\xi_{l}, k_{\perp}\right) \equiv & e^{-2 a q_{l}}\left[\left(2 q_{l}^{2}-\frac{\xi_{l}^{2}}{c^{2}}\right) r_{\mathrm{TM}}\left(i \xi_{l}, k_{\perp}\right)\right. \\
& \left.-\frac{\xi_{l}^{2}}{c^{2}} r_{\mathrm{TE}}\left(i \xi_{l}, y\right)\right]
\end{aligned}
$$

The reflection coefficient $r_{\mathrm{TM}}\left(0, k_{\perp}\right)$ in Eq. (22) takes different values for different wall materials. For dielectrics with finite static dielectric permittivity $\varepsilon_{0}$ we get $r_{\mathrm{TM}}\left(0, k_{\perp}\right)=\left(\varepsilon_{0}-1\right) /\left(\varepsilon_{0}+1\right)$, and for metals $r_{\mathrm{TM}}\left(0, k_{\perp}\right)=1$. 
At zero temperature Eq. (21) results in

$$
E(a)=-\frac{\hbar}{2 \pi} \int_{0}^{\infty} d \xi \alpha(i \xi) \int_{0}^{\infty} \frac{k_{\perp} d k_{\perp}}{q} \Phi_{A}\left(\xi, k_{\perp}\right) .
$$

In the nonrelativistic limit Eq. (23) leads to the wellknown result (Lifshitz and Pitaevskii, 1980),

$$
E(a) \approx-\frac{\hbar}{4 \pi a^{3}} \int_{0}^{\infty} \alpha(i \xi) \frac{\varepsilon(i \xi)-1}{\varepsilon(i \xi)+1} d \xi \equiv-\frac{C_{3}}{a^{3}} .
$$

If we consider an atom characterized by the frequencyindependent (static) polarizability $\alpha\left(i \xi_{l}\right)=\alpha(0)$ near an ideal metal wall at $T=0$, Eq. (23) results in

$$
E(a)=-\frac{3 \hbar c}{8 \pi a^{4}} \alpha(0) \equiv-\frac{C_{4}}{a^{4}} .
$$

This is the famous result obtained by Casimir and Polder (1948).

Calculations performed using the exact expression (21) showed that the approximation [Eq. (24)] is valid only at very short separation distances $a<3 \mathrm{~nm}$. To calculate $C_{3}$ in this case one should use the highly accurate $N$-oscillator model (Shih and Parsegian, 1975),

$$
\alpha(i \xi)=\frac{e^{2}}{m} \sum_{n=1}^{N} \frac{f_{0 n}}{\omega_{0 n}^{2}+\xi^{2}},
$$

for the dynamic polarizability of an atom (Caride et al., 2005). In Eq. (26) $m$ and $e$ are the electron mass and charge and $f_{0 n}$ and $\omega_{0 n}$ are the oscillator strength and transition frequency from the $n$th excited state to the ground-state transition, respectively. With the increase in the atom-wall distance the retardation effects become important. Starting from distances of several tens of nanometers, however, a more simplified single-oscillator model for the atomic polarizability is applicable. The description of an atom with the help of a frequencyindependent static polarizability works well only at rather large separation distances $a>2 \mu \mathrm{m}$ (Babb et al., 2004). At room temperature, however, the temperature corrections in Eq. (21) come into play at $a \geqslant 3 \mu \mathrm{m}$. Thus, the approximation (25) at room temperature works well only within a rather narrow separation region from 2 to $3 \mu \mathrm{m}$.

From Eq. (21), the Casimir-Polder force acting on an atom situated near a wall a distance $a$ apart can be represented in the form (Babb et al., 2004)

$$
F(a, T)=-2 k_{B} T \sum_{l=0}^{\infty} \alpha\left(i \xi_{l}\right) \int_{0}^{\infty} k_{\perp} d k_{\perp} \Phi_{A}\left(\xi_{l}, k_{\perp}\right) .
$$

Calculations of the energy of atom-wall interactions and of the Casimir-Polder force play an important role for the interpretation of experiments on quantum reflection, i.e., above-barrier reflection of slow atoms, with incident kinetic energy exceeding the barrier height [see, e.g., Côté et al. (1998), Druzhinina and DeKieviet (2003), Pasquini et al. (2004), Oberst, Kouznetsov, et al. (2005);
Oberst, Taashiro, et al. (2005), and references therein]. Results on Casimir-Polder forces from such experiments are discussed in Sec. VI.B.

The Casimir-Polder interaction leads also to a change of center-of-mass oscillation frequency $\omega_{0}$ of a BoseEinstein condensate (Antezza et al., 2004),

$$
\begin{aligned}
\omega_{0}^{2}-\omega_{z}^{2}= & -\frac{\omega_{0}}{\pi A m_{a}} \int_{0}^{2 \pi / \omega_{0}} d \tau \cos \left(\omega_{0} \tau\right) \\
& \times \int_{-R_{z}}^{R_{z}} d z n_{z}(z) F\left[a+z+A \cos \left(\omega_{0} \tau\right), T\right] .
\end{aligned}
$$

Here the Casimir-Polder force in thermal equilibrium is given by Eq. (27); $m_{a}$ is the mass of atoms of the BoseEinstein condensate under consideration. The averaging procedure includes the averaging of time over the period of oscillations in the $z$ direction with an amplitude $A$ ( $z$ is perpendicular to the plate) and the density of gas with a distribution function

$$
n_{z}(z)=\frac{15}{16 R_{z}}\left(1-\frac{z^{2}}{R_{z}^{2}}\right)^{2},
$$

where $R_{z}$ is the Thomas-Fermi radius in the $z$ direction.

The measurement of the change in the center-of-mass oscillation frequency (Harber et al., 2005; Obrecht et al., 2007) is a sensitive test of the Casimir-Polder force. Results of the recent experiment (Obrecht et al., 2007), where the thermal effect was measured for the first time in Casimir physics, are discussed in Sec. VI.A.

\section{Puzzles in the application of the Lifshitz theory to real materials}

\section{Real metals}

In the framework of the Lifshitz theory of the van der Waals and Casimir forces, discussed above, the calculational results depend strongly on the model of dielectric permittivity used to describe real material. Different problems arise for metals and dielectrics. The source of the discrepancy is in the different contributions from the zero frequency term [i.e., from the term with $l=0$ in Eq. (3)]. For metals, problems result from the contribution of the transverse electric mode. For ideal metal plates the Casimir free energy was found independently of the Lifshitz theory within the frames of thermal quantum field theory with electrodynamic boundary conditions $E_{t}=B_{n}=0$ on the surface of plates (Mehra, 1967; Brown and Maclay, 1969). As mentioned in the Introduction, the Lifshitz theory agrees with the case of an ideal metal if one takes the limit of infinite dielectric permittivity, $\varepsilon\left(i \xi_{l}\right)=\infty$, before putting the frequency equal to zero in the temperature sum [Eq. (3)] (Schwinger et al., 1978). Then from Eqs. (6) and (7) one obtains

$$
r_{\mathrm{TM}}\left(i \xi_{l}, k_{\perp}\right)=1, \quad r_{\mathrm{TE}}\left(i \xi_{l}, k_{\perp}\right)=-1
$$

for any $l$ including $l=0$. After the substitution into Eq. (3) this leads to the same result for the Casimir free 
energy, as obtained independently using thermal quantum field theory. The sequence of limiting transitions used to obtain Eq. (30) is called the Schwinger prescription.

The ideal metal, however, can be obtained as the limiting case of real metals when the conductivity goes to infinity. It is well known (Landau et al., 1984) that for real metals $\varepsilon(i \xi) \sim 1 / \xi$ when $\xi \rightarrow 0$ (the Drude model is one particular case). Then from Eq. (6) we get for real metals

$$
r_{\mathrm{TM}}\left(0, k_{\perp}\right)=1, \quad r_{\mathrm{TE}}\left(0, k_{\perp}\right)=0 .
$$

In the limit of infinite conductivity one again obtains Eq. (30) for the reflection coefficients with $l \geqslant 1$, but for the reflection coefficients with $l=0 \mathrm{Eq}$. (31) remains valid. This would lead to a different result for ideal metals than is obtained from the Schwinger prescription. As shown below, this contradiction arises from the use of the dielectric permittivity $\sim 1 / \xi$ which is outside the applicability conditions of the Lifshitz theory.

Conceptually the Lifshitz theory provides a way for obtaining all the necessary results for any real material. One may describe the free electrons in metals by the plasma model [as suggested in the first papers devoted to the calculation of the finite conductivity corrections to the Casimir result (Lifshitz, 1956; Hargreaves, 1965; Schwinger et al., 1978)],

$$
\varepsilon(\omega)=1-\omega_{p}^{2} / \omega^{2} .
$$

Here $\omega_{p}$ is the plasma frequency. The dielectric permittivity (32) is applicable in the frequency region of infrared optics (Lifshitz and Pitaevskii, 1981). At room temperature all nonzero Matsubara frequencies belong to this region. Because of this, the plasma model leads to rather accurate results for the Casimir force at separation distances above the plasma wavelength. Below, the generalized plasmalike dielectric permittivity is also considered, which takes into account dissipation due to interband transitions of core electrons and can be applied at shorter separations (see Sec. III.A.1). The results obtained for real metals using the Lifshitz theory with the permittivity (32) are qualitatively close to those obtained for ideal metals using thermal quantum field theory (Bordag et al., 2000b; Genet et al., 2000). Thus, in the low-temperature limit $T \ll T_{\text {eff }} \quad\left(k_{B} T_{\text {eff }}=\hbar c / 2 a\right)$, the asymptotic expression for the thermal correction to the energy for two similar plates, as defined in Eq. (15), is given by (Bordag et al., 2000b; Geyer et al., 2001)

$$
\begin{aligned}
\Delta_{T} \mathcal{F}^{(p)}(a, T)= & -\frac{\pi^{2} \hbar c}{720 a^{3}}\left\{1+\frac{45 \zeta(3)}{\pi^{3}}\left(\frac{T}{T_{\text {eff }}}\right)^{3}-\left(\frac{T}{T_{\text {eff }}}\right)^{4}\right. \\
& +\frac{2 c}{\omega_{p} a}\left[\frac{45 \zeta(3)}{\pi^{3}}\left(\frac{T}{T_{\text {eff }}}\right)^{3}-\frac{1}{2}\left(\frac{T}{T_{\text {eff }}}\right)^{4}\right] \\
& +\cdots\} .
\end{aligned}
$$

Here $\zeta(z)$ is the Riemann zeta function. Note that in this case the quantity $\Delta \mathcal{F}(a, T)$, as defined in Eq. (14), coincides with the thermal correction (15). For an ideal metal $\omega_{p} \rightarrow \infty$ and Eq. (33) reproduces the lowtemperature asymptotic behavior obtained with thermal quantum field theory (Brown and Maclay, 1969). In the high-temperature limit $T \gg T_{\text {eff }}$ (at room temperature $T$ $=300 \mathrm{~K}$ it is achieved at $a \geqslant 6 \mu \mathrm{m})$ the asymptotic expression for the Casimir free energy is (Bordag et al., 2000b)

$$
\mathcal{F}(a, T)=-\frac{k_{B} T \zeta(3)}{8 \pi a^{2}}\left(1-\frac{2 c}{\omega_{p} a}+\cdots\right) .
$$

Here $c / \omega_{p}=\delta_{0}$ is the skin depth and $\delta_{0} / a$ is a small parameter. The finite conductivity correction in Eq. (34) is negligibly small. Thus, the high-temperature limit for real metals described by the plasma model dielectric permittivity (32) coincides with that obtained for ideal metals (Brown and Maclay, 1969; Feinberg et al., 2001). The agreement between the Lifshitz theory combined with the plasma model and the case of ideal metals, as described by thermal quantum field theory, has a simple explanation. It is because for the plasma model with $\omega_{p} \rightarrow \infty$, it is not Eq. (31) but Eq. (30) that is satisfied for the reflection coefficients at zero frequency.

A different situation occurs when the Drude dielectric function

$$
\varepsilon_{D}(\omega)=1-\omega_{p}^{2} / \omega[\omega+i \gamma(T)],
$$

where $\gamma(T)$ is the relaxation parameter, is substituted into the Lifshitz formula (Boström and Sernelius, 2000; Høye et al., 2003, 2006; Brevik et al., 2005). In this case the zero-frequency values of the reflection coefficients are given by Eq. (31), and the high-temperature limit is equal to one-half of the corresponding value for ideal metals, regardless of how large a conductivity is used for the real metal (Klimchitskaya and Mostepanenko, 2001).

The low-temperature thermal correction, calculated with the dielectric permittivity (35), can be presented in the form (Bezerra et al., 2004)

$$
\begin{aligned}
\Delta_{T} \mathcal{F}^{(D)}(a, T) & \\
= & \Delta_{T} \mathcal{F}^{(p)}(a, T)+\mathcal{F}^{(\gamma)}(a, T)-\frac{k_{B} T}{16 \pi a^{2}} \int_{0}^{\infty} y d y \\
& \times \ln \left[1-\left(\frac{c y-\sqrt{4 a^{2} \omega_{p}^{2}+c^{2} y^{2}}}{c y+\sqrt{4 a^{2} \omega_{p}^{2}+c^{2} y^{2}}}\right)^{2} e^{-y}\right] .
\end{aligned}
$$

With the condition that

$$
\gamma(T)<\xi_{1},
$$

the low-temperature behavior of the second term on the right-hand side of Eq. (36) is $\mathcal{F}^{(\gamma)}(a, T) \sim \gamma(T) \ln \left(T / T_{\text {eff }}\right)$ (Bezerra et al., 2004). For metals with perfect crystal lattices $\gamma(T) \rightarrow 0$ as $T^{2}$ (Lifshitz et al., 1973) and the inequality (37) is satisfied down to $T=0$. For metals with impurities there exists some residual value $\gamma_{\text {res }} \neq 0$ (Kittel, 1996). As a result, at a $T$ of about $10^{-4} \mathrm{~K}$ typical residual relaxation becomes equal to $\xi_{1}$ and for smaller $T$ Eq. (37) is violated.

For room temperature, at separation distances below $1 \mu \mathrm{m}$ the third term on the right-hand side of Eq. (36) is 
dominant. It describes a rather large linear-intemperature thermal correction to the Casimir free energy and pressure, as predicted by the Drude model. This thermal correction is positive, i.e., it decreases the magnitude of the Casimir free energy and pressure. As shown in Sec. IV.B, such a thermal correction is excluded by experiments at a high confidence level.

From a theoretical basis the thermal correction (36) is not appropriate because it is inconsistent with thermodynamics for metals with perfect crystal lattices. To verify the consistency with thermodynamics, one should consider the Casimir entropy (Bezerra et al., 2002a, 2002b, 2004)

$$
S(a, T)=-\partial \mathcal{F}(a, T) / \partial T .
$$

In the case of metals described by the plasma model dielectric permittivity (32) $S^{(p)}(a, T) \sim T^{2} \rightarrow 0$ when $T$ $\rightarrow 0$ (Bezerra et al., 2002b). In the case of Drude metals with perfect crystal lattices, the Casimir entropy has a nonzero negative value at $T=0$ following from Eq. (36) (Bezerra et al., 2002a, 2002b, 2004, 2006; Mostepanenko, Bezerra, et al., 2006)

$$
\begin{aligned}
S^{(D)}(a, 0)= & \frac{k_{B}}{16 \pi a^{2}} \int_{0}^{\infty} y d y \\
& \times \ln \left[1-\left(\frac{c y-\sqrt{4 a^{2} \omega_{p}^{2}+c^{2} y^{2}}}{c y+\sqrt{4 a^{2} \omega_{p}^{2}+c^{2} y^{2}}}\right)^{2} e^{-y}\right] \\
= & -\frac{k_{B} \zeta(3)}{16 \pi a^{2}}\left(1-\frac{4 c}{\omega_{p} a}+12 \frac{c^{2}}{\omega_{p}^{2} a^{2}}-\cdots\right) \\
< & 0 .
\end{aligned}
$$

Note that the use of the Drude model in the Lifshitz formula is usually justified by the fact that it provides smooth transition between the region of the normal skin effect and that of infrared optics. This ignores the region of the anomalous skin effect where the Lifshitz formula in terms of $\varepsilon(\omega)$ is not applicable. With the decrease of $T$ the application region of the normal skin effect becomes more narrow and the application region of the anomalous skin effect widens. However, at any $T>0$, there exists a frequency region including zero frequency where the normal skin effect is applicable. Thus, it is worthwhile to use the Drude model at low $T$ when discussing the thermodynamic consistency of the Lifshitz theory.

It was shown (Boström and Sernelius, 2004; Høye et al., 2007; Brevik et al., 2008) that for Drude metals with impurities the Casimir entropy jumps abruptly to zero at $T<10^{-4} \mathrm{~K}$ starting from a negative value [Eq. (39)]. The corresponding analytic expression for the lowtemperature behavior of the entropy has been obtained (Høye et al., 2007; Brevik et al., 2008) under the condition $\zeta_{l} \ll \gamma_{\text {res }}$, which is opposite to the inequality (37). The application region and the coefficients of this analytic expression were determined incorrectly because an overestimated value of $\gamma_{\mathrm{res}}=\gamma(T=300 \mathrm{~K})$ was used. The correct values of the coefficients were found by Klimchitskaya and Mostepanenko (2008a), who also demon- strated that the application region of the obtained expressions is at temperatures below $T=10^{-5} \mathrm{~K}$.

According to Høye et al. (2007) and Brevik et al. (2008), the formal satisfaction of the Nernst theorem for the Drude model, as applied to metals with impurities, resolves the problem of thermodynamic consistency of that model combined with the Lifshitz theory. Klimchitskaya and Mostepanenko (2008a) remarked that a perfect crystal lattice is a truly equilibrium system with a nondegenerate dynamical state of the lowest energy. At zero temperature any part of the system must be in the ground quantum state (Landau and Lifshitz, 1980; Rumer and Ryvkin, 1980). As a result, the Casimir entropy computed for a perfect crystal lattice must be equal to zero, a direct consequence of quantum statistical physics. Thus, they pointed out that the Drude model combined with the Lifshitz formula violates the Nernst heat theorem for a perfect crystal lattice and is in contradiction with quantum statistical physics.

Høye et al. (2008) argued that the Drude model combined with the Lifshitz formula is thermodynamically consistent in the case of a perfect crystal lattice also. To justify this statement they introduced the definition of a perfect lattice as the limiting case of a crystal lattice with nonzero residual relaxation $\gamma_{\text {res }}$ when $\gamma_{\text {res }} \rightarrow 0$. However, in condensed matter physics (Kittel, 1996), a perfect lattice is commonly defined as a lattice with perfect crystal structure without impurities, i.e., with $\gamma_{\text {res }}=0$ from the outset. This common definition allows one to develop the quantum theory of electron-phonon interactions, which is based on the symmetry properties of the lattice. Specifically, the relaxation parameter turns out to be temperature dependent and vanishes with vanishing temperature. The definition of a perfect lattice introduced by Høye et al. (2008) violates lattice symmetry properties. For example, it would not be possible to reproduce the standard results of the theory of elementary excitations on the basis of this definition. Recently, Intravaia and Henkel (2008) independently verified that the Drude model used in the Lifshitz formula violates the Nernst heat theorem for metals with perfect crystal lattices as commonly understood $[\gamma(T) \rightarrow 0$ at $T \rightarrow 0]$, in accordance with original statement by Bezerra et al. (2002a, 2002b, 2004).

Note that the substitution of the Drude dielectric function (35) into the Lifshitz formula (3) for metal plates of finite size is somewhat contradictory (Parsegian, 2005; Geyer et al., 2007; Mostepanenko and Geyer, 2008). As pointed out by Parsegian (2005), the Drude model is derived from the Maxwell equations applied to an infinite metallic medium (semispace) with no external sources, with zero induced charge density, and with nonzero drift current. In such a medium there are no walls limiting the flow of charges. For real metal plates of finite size, however, the applicability conditions of the Drude model are violated. If one admits that the electric field of the zero-point oscillations with vanishingly small frequencies (almost zero) creates a short-lived current of conduction electrons, this leads to the formation of almost constant charge densities $\pm \Sigma$ on the sidewalls of 
the Casimir plates. As a result, both the electric field and the current inside the plate will vanish due to screening, in a very short time interval of order of $10^{-18} \mathrm{~s}$ (Geyer et al., 2007). Because of this behavior, for electromagnetic fields at very low frequency, the finite metal plates should be described not by the Drude dielectric function (35), but by the plasma model, which allows for displacement currents only (see Sec. III.A). The role of finite size effects of the conductors was also illustrated in the case of two wires of finite length described by the Drude model and interacting through the inductive coupling between Johnson currents (Bimonte, 2007). It was shown that in the thermal interaction between the wires the Nernst theorem is followed or violated depending on whether the capacitive effects associated with the end points of the wires are taken or not taken into account.

Another approach to the resolution of the puzzles arising in the application of the Lifshitz theory to real materials includes the effect of screening on the reflection coefficients (Dalvit and Lamoreaux, 2008). This approach takes into account both the drift and diffusion currents of free charge carriers through the use of the transport Boltzmann equation. It uses the standard Lifshitz formulas (3) and (21) for the free energy of wallwall and atom-wall interactions with the TE reflection coefficient $r_{\mathrm{TE}}$ as defined in Eq. (6) within the Drude model approach, but with the modified TM reflection coefficient

$$
r_{\mathrm{TM}}^{\bmod }\left(i x, k_{\perp}\right)=\frac{\varepsilon(i x) q-k-\frac{k_{\perp}^{2}}{\eta(i x)} \frac{\varepsilon(i x)-\varepsilon_{c}(i x)}{\varepsilon_{c}(i x)}}{\varepsilon(i x) q+k+\frac{k_{\perp}^{2}}{\eta(i x)} \frac{\varepsilon(i x)-\varepsilon_{c}(i x)}{\varepsilon_{c}(i x)}} .
$$

Here the dielectric permittivity is given by

$$
\varepsilon(i x)=\varepsilon_{c}(i x)+\omega_{p}^{2} / x(x+\gamma),
$$

where $\varepsilon_{c}(i x)$ is the permittivity of the bound core electrons,

$$
\varepsilon_{c}(i x)=1+\sum_{j} \frac{g_{j}}{\omega_{j}^{2}+x^{2}+\gamma_{j} x},
$$

with nonzero oscillator frequencies $\omega_{j} \neq 0$, oscillator strengths $g_{j}$, and damping parameters $\gamma_{j}$. The quantity $\eta(i x)$ is defined as

$$
\eta(i x)=\left(k_{\perp}^{2}+\kappa^{2} \frac{\varepsilon_{c}(0)}{\varepsilon_{c}(i x)} \frac{\varepsilon(i x)}{\varepsilon(i x)-\varepsilon_{c}(i x)}\right)^{1 / 2},
$$

where $1 / \kappa$ is the screening length. If the charge carriers of density $n$ are described by the classical MaxwellBoltzmann statistics [Dalvit and Lamoreaux (2008)], one gets the Debye-Hückel screening length $R_{D}=1 / \kappa$ $=\left(\varepsilon_{0} k_{B} T / 4 \pi e^{2} n\right)^{1 / 2}$. Assuming Fermi-Dirac statistics, one arrives at the Thomas-Fermi screening length $R_{\mathrm{TF}}$ $=1 / \kappa=\left(\varepsilon_{0} E_{F} / 6 \pi e^{2} n\right)^{1 / 2} \quad($ Landau and Lifshitz, 1980; Chazalviel, 1999). Here $e$ is the electron charge, $\varepsilon_{0}$ $=\varepsilon_{c}(0)$ is the dielectric constant due to the core electrons, and $E_{F}=\hbar \omega_{p}$ is the Fermi energy. It was shown, however, that the modification of the TM reflection co- efficient in accordance with Eq. (40) leads to the violation of the Nernst heat theorem for those dielectric materials whose charge carrier density does not vanish when $T \rightarrow 0$ and conductivity vanishes due to the vanishing mobility (Klimchitskaya, Mohideen, and Mostepanenko, 2008; Klimchitskaya, 2009). For metals with perfect crystal lattices this approach violates the Nernst theorem as discussed above (Mostepanenko et al., 2009). Note that Dalvit and Lamoreaux (2008) applied their approach to intrinsic semiconductor media. Bearing in mind, however, that the classical Drude dielectric permittivity is exploited, the method can be applied for metals and doped semiconductors as well by substituting the corresponding screening length. As shown in Secs. IV.B and V.B, the computational results using the modified TM reflection coefficient are excluded by the measurement data of two precise experiments (Chen et al., 2007a, 2007b; Decca et al., 2007a, 2007b).

As shown above, the description of real metals at low frequencies by the seemingly obvious dielectric permittivity of the Drude model leads to serious difficulties. Attempts at solving this problem by taking into account the screening effects due to finite size of the plates or diffusion currents of free charge carriers also failed. The latter approach, if successful, would in fact be a recognition that the standard reflection coefficients, as used in the Lifshitz theory, expressed in terms of the dielectric permittivity are incorrect and one of them must be modified using an additional microscopic quantity, the density of free charge carriers.

The origin of the problem can be understood if one takes into consideration the fact that the inclusion of the drift current, as described by the Drude model, violates the basic applicability condition of the Lifshitz theory. This is the demand that the dielectric media and the fluctuating electromagnetic field should be in thermal equilibrium with the heat reservoir. The point is that the existence of the drift electric current leads to a violation of time-reversal symmetry and the introduction of the interaction between the system and the heat reservoir violating the state of thermal equilibrium (Bryksin and Petrov, 2008). This is seen from the fact that the Drude model is derived from Maxwell equations with the drift current of conduction electrons on the right-hand side (see above). In its turn, the drift current leads to the emergence of Joule losses, i.e., to a heating of the crystal lattice (Geyer et al., 2003). In order to preserve the temperature constant, the unidirectional flux of heat from the Casimir plates to the heat reservoir must inevitably arise. Such an interaction between the system and the heat reservoir cannot exist in the state of thermal equilibrium as it is in contradiction with its definition (Rumer and Ryvkin, 1980; Kondepudi and Prigogine, 1998).

Thus not just any dielectric permittivity can be considered in combination with the Lifshitz formula. Specifically, the dielectric permittivity of the Drude model (or any other that is inversely proportional to the first power of the frequency at low frequencies) does not belong to the application region of the Lifshitz theory. In a similar 
way, the approach based on the transport Boltzmann equation, in addition to the drift current, introduces the diffusion current determined by a nonequilibrium distribution of free charge carriers. This exacerbates the violation of thermal equilibrium. Thus, the modified reflection coefficient (40) obtained from this approach cannot be substituted into the Lifshitz formula. On the other hand, the dielectric permittivity of the plasma model [Eq. (32)] and its generalized version taking into account the interband transitions [see Eq. (58) in Sec. III.A.1] do not admit the drift current but only the displacement one. Thus, they are compatible with the demand of thermal equilibrium and can be substituted into the Lifshitz formulas.

\section{Real dielectrics}

As mentioned in the Introduction, puzzling results were obtained in the application of the Lifshitz theory to real dielectrics. It is well known that all dielectric materials (insulators and intrinsic semiconductors characterized by a band gap of different width, Mott-Hubbard dielectrics, and doped semiconductors with doping concentration below the critical value) have a zero value of the dc conductivity at zero temperature [see, e.g., Shklovskii and Efros (1984) and Mott (1990)]. At nonzero temperature these materials have a nonzero value of dc conductivity. Sometimes this conductivity is rather large, as, for instance, for doped semiconductors with a doping concentration only slightly below the critical value. In the commonly used application of the Lifshitz theory the dc conductivity of dielectrics is neglected. Their conductivity is assumed to be equal to zero at any temperature. In this case the dielectric permittivity is represented in the form [see, e.g., Parsegian (2005)]

$$
\varepsilon(\omega)=\varepsilon_{c}(\omega)=1+\sum_{j} \frac{g_{j}}{\omega_{j}^{2}-\omega^{2}-i \gamma_{j} \omega},
$$

where $\omega_{j} \neq 0$ are the oscillator frequencies, $g_{j}$ are the oscillator strengths, and $\gamma_{j}$ are the damping parameters [note that when $\varepsilon(i \xi)$ is calculated along the imaginary frequency axis the damping parameters are often neglected and some approximate formulas are used (Hough and White, 1980; Bergström, 1997; Parsegian, 2005)]. From Eq. (44), the dielectric permittivity at zero frequency is given by

$$
\varepsilon_{0} \equiv \varepsilon(0)=1+\sum_{j} \frac{g_{j}}{\omega_{j}^{2}}<\infty
$$

and the reflection coefficients at zero frequency are

$$
r_{\mathrm{TM}}\left(0, k_{\perp}\right)=\frac{\varepsilon_{0}-1}{\varepsilon_{0}+1} \equiv r_{0}, \quad r_{\mathrm{TE}}\left(0, k_{\perp}\right)=0 .
$$

However, if one takes into account the existence of free charge carriers at nonzero temperature, the dielectric permittivity can be represented by

$$
\tilde{\varepsilon}(\omega, T)=\varepsilon(\omega)+i 4 \pi \sigma_{0}(T) / \omega,
$$

where $\sigma_{0}(T)$ is the dc conductivity at $T \neq 0$ and $\varepsilon(\omega)$ is given by Eq. (44) (Palik, 1985; Zurita-Sánches et al., 2004). Then, instead of Eq. (46), the reflection coefficients at $\xi=0$ are given by Eq. (31).

The analysis of the low-temperature asymptotic behavior of the Casimir free energy (3) calculated with the dielectric permittivity (44) for two similar plates shows that (Geyer et al., 2005b, 2006; Klimchitskaya and Geyer, 2008)

$$
\begin{aligned}
\mathcal{F}(a, T)= & E(a)-\frac{\hbar c}{8 a^{3}}\left[\frac{b(a) \mathrm{Li}_{2}\left(r_{0}^{2}\right)}{3\left(\varepsilon_{0}^{2}-1\right)}\left(\frac{T}{T_{\text {eff }}}\right)^{2}\right. \\
& \left.+\frac{\zeta(3) r_{0}^{2}\left(\varepsilon_{0}+1\right)}{4 \pi}\left(\frac{T}{T_{\text {eff }}}\right)^{3}\right],
\end{aligned}
$$

where $\operatorname{Li}_{n}(z)$ is the polylogarithm function, and the following notation is used:

$$
b(a)=\sum_{j} \frac{g_{j} \gamma_{j} c}{2 a \omega_{j}^{4}} .
$$

From Eq. (48) the Casimir entropy is given by

$$
\begin{aligned}
S(a, T)= & \frac{k_{B}}{4 a^{2}} \frac{T}{T_{\text {eff }}}\left(\frac{2 b(a) \mathrm{Li}_{2}\left(r_{0}^{2}\right)}{3\left(\varepsilon_{0}^{2}-1\right)}\right. \\
& \left.+\frac{3 \zeta(3) r_{0}^{2}\left(\varepsilon_{0}+1\right)}{4 \pi} \frac{T}{T_{\text {eff }}}\right) .
\end{aligned}
$$

As is seen from Eq. (50), $S(a, T)$ goes to zero when $T$ $\rightarrow 0$, i.e., the Nernst heat theorem is satisfied when the dielectric permittivity is given by Eq. (44) with a finite static value [Eq. (45)].

A completely different result is obtained if one takes into account the conductivity of dielectrics at zero frequency that arises at $T>0$. Note that in all cases this conductivity vanishes exponentially, $\sigma_{0}(T) \sim \exp (-C / T)$, when $T \rightarrow 0$ (Shklovskii and Efros, 1984; Mott, 1990). The reflection coefficients at zero frequency calculated with the dielectric permittivity (47) do not satisfy Eq. (46) but Eq. (31). This means that the TM reflection coefficient has a discontinuity when the dc conductivity of the dielectric material is taken into account. Substituting the dielectric permittivity (47) into the Lifshitz formula (3), we arrive at (Geyer et al., 2005b)

$$
\tilde{\mathcal{F}}(a, T)=\mathcal{F}(a, T)-\frac{k_{B} T}{16 \pi a^{2}}\left[\zeta(3)-\operatorname{Li}_{3}\left(r_{0}^{2}\right)\right]+R(T),
$$

where the low-temperature behavior of $\mathcal{F}(a, T)$ is given by Eq. (48) and $R(T)$ decreases exponentially when $T$ vanishes.

From Eq. (51), the Casimir entropy at $T=0$ is given by

$$
\tilde{S}(a, 0)=\left(k_{B} / 16 \pi a^{2}\right)\left[\zeta(3)-\mathrm{Li}_{3}\left(r_{0}^{2}\right)\right]>0,
$$

in violation of the Nernst theorem. Thus, the inclusion of a nonzero conductivity arising at $T>0$ into the model of the dielectric response leads to a contradiction between the Lifshitz theory and thermodynamics for all 
materials which have a zero conductivity at zero temperature, i.e., for all dielectrics. The same happens if we include the dc conductivity of the dielectric at $T>0$ when describing the Casimir force between a metal and a dielectric plate (Geyer et al., 2005a, 2006; Klimchitskaya, Geyer, and Mostepanenko, 2006; Geyer, Klimchitskaya, and Mostepanenko, 2008). In this case the Casimir entropy at $T=0$ is given by Eq. (52) where $\mathrm{Li}_{3}\left(r_{0}^{2}\right)$ should be replaced with $\operatorname{Li}_{3}\left(r_{0}\right)$. The above puzzling results are in line with those obtained for real metals described by the Drude model. They are explained by the fact that the dielectric permittivity (47) is outside the application region of the Lifshitz theory. Thus, the nonzero conductivity arising at $T>0$ for all dielectric materials must be disregarded in theoretical computations using the Lifshitz formula (3). This rule has already been confirmed experimentally (see Secs. V.B and VI.A).

Similarly to the case of two dielectric plates, for an atom near a dielectric wall the Lifshitz theory is thermodynamically consistent if the dc conductivity is neglected. However, the inclusion of the dc conductivity of the wall material in accordance with Eq. (47) leads to the violation of the Nernst heat theorem (Klimchitskaya, Mohideen, and Mostepanenko, 2008). An attempt to solve this problem beyond the scope of the standard Lifshitz theory was undertaken by Pitaevskii (2008). This attempt makes use of the effect of Debye-Hückel screening mentioned above in connection with metals. The effect of screening is taken into account only for the static field. It leads to the replacement of the transverse magnetic reflection coefficient at zero frequency, as given by Eq. (46), with

$$
r_{\mathrm{TM}}^{\mathrm{mod}}\left(0, k_{\perp}\right)=\left(\varepsilon_{0} \sqrt{k_{\perp}^{2}+\kappa^{2}}-k_{\perp}\right) /\left(\varepsilon_{0} \sqrt{k_{\perp}^{2}+\kappa^{2}}+k_{\perp}\right),
$$

where $\kappa=1 / R_{D}$ is defined in Sec. II.D.1. When the total density of charge carriers $n=0$, Eq. (53) leads to the same result as Eq. (46). For $n \rightarrow \infty$, at fixed $T \neq 0$, $r_{\mathrm{TM}}^{\bmod }\left(0, k_{\perp}\right)=1$, as in the standard Lifshitz theory with the inclusion of the dc conductivity. This solves the peculiarity of the small- $\sigma$ limit, i.e., the abrupt jump from Eq. (46) to Eq. (31) arising from an arbitrarily small conductivity. According to Pitaevskii (2008), "the abovementioned peculiarity of the small $\sigma$ limit exists only for the $l=0$ term." This permits one to assume that all the coefficients $r_{\mathrm{TM}, \mathrm{TE}}\left(i \xi_{l}, k_{\perp}\right)$ with $l \geqslant 1$ under some conditions remain unchanged. This assumption was confirmed by Dalvit and Lamoreaux (2008), who obtained both the TM and TE reflection coefficients with account of the screening effects at arbitrary Matsubara frequencies. The TE reflection coefficient was found to coincide with the standard one obtained using the Drude model approach and the modified TM reflection coefficient is given by Eq. (40). It is easily seen that for materials with sufficiently small charge carrier density (for instance, for Si with $n \leqslant 10^{17} \mathrm{~cm}^{-3}$ ) the modified reflection coefficient [Eq. (40)] at room temperature $T=300 \mathrm{~K}$ takes approximately the same values as the standard one in Eq. (6) at all nonzero Matsubara frequencies. If one takes into account the fact that the reflection coefficient (53) obtained by Pitaevskii (2008) is just the reflection coefficient (40) at $x=0$, this concludes the proof of the above assumption.

The reflection coefficient (53) can be obtained by the formal replacement of the dielectric permittivities depending only on frequency with the permittivities depending on both frequency and wave vector in the Fresnel reflection coefficient of an uniaxial crystal. To confirm that this is the case, one should introduce dissimilar permittivities $\varepsilon_{x}=\varepsilon_{y}$ and $\varepsilon_{z}$ and replace the coefficient $r_{\mathrm{TM}}$ in Eq. (6) with

$$
r_{\mathrm{TM}}^{\bmod }\left(i x, k_{\perp}\right)=\frac{\sqrt{\varepsilon_{x}(i x) \varepsilon_{z}(i x)} q-k_{z}}{\sqrt{\varepsilon_{x}(i x) \varepsilon_{z}(i x)} q+k_{z}} .
$$

Here $k_{z}$ is defined by Eq. (7) with the replacement of $\varepsilon(i x)$ for $\varepsilon_{z}(i x)$. With $\varepsilon_{x}$ and $\varepsilon_{z}$ depending only on the frequency, Eq. (54) is commonly used for the description of uniaxial crystals (Greenaway et al., 1969; Blagov et al., 2005). Then Eq. (53) follows from Eq. (54) if one sets

$$
\varepsilon_{x}(0)=\varepsilon_{0}, \quad \varepsilon_{z}(0)=\varepsilon_{z}\left(0, k_{\perp}\right)=\varepsilon_{0}\left(1+\kappa^{2} / k_{\perp}^{2}\right),
$$

where now $\varepsilon_{z}(0)$ depends on the wave vector. Thus, to obtain Eq. (53) one substitutes into the reflection coefficient (54) derived from the standard continuity boundary conditions the dielectric permittivity depending on a wave vector (see Sec. III.A.3 for reasons why this is inappropriate).

It was verified (Klimchitskaya, Mohideen, and Mostepanenko, 2008) that for pure insulators and intrinsic semiconductors [i.e., for materials for which $n(T)$ exponentially decays to zero with vanishing temperature] the Lifshitz theory with the modified reflection coefficient (53) satisfies the Nernst heat theorem. However, if $n$ does not go to zero when $T$ goes to zero (this is true, for instance, for dielectric materials, such as semiconductors doped below critical doping concentration or solids with ionic conductivity), the Lifshitz theory with the reflection coefficient (53) leads to a positive CasimirPolder entropy at $T=0$, i.e., violates the Nernst heat theorem (Klimchitskaya, Mohideen, and Mostepanenko, 2008). Note here that the entropy of the fluctuating electromagnetic field is nonzero at $T=0$ and depends on separation, whereas the entropy of the plates is separation independent. Because of this, the entropy of the plates at $T=0$ cannot compensate the nonzero entropy of the fluctuating field so as to make the total entropy independent of the parameters of the system in accordance with the Nernst theorem. In fact, the conductivity is given by $\sigma_{0}(T)=n|e| \mu$, where $\mu$ is the mobility of charge carriers (Ashcroft and Mermin, 1976). Although $\sigma_{0}(T)$ goes to zero exponentially fast for all dielectrics when $T$ goes to zero, for most of them this happens not due to the vanishing $n$ but due to the vanishing mobility. One such example is fused silica $\left(\mathrm{SiO}_{2}\right)$ considered in Sec. VI.A in connection with experiments on the Casimir-Polder force. Its conductivity is ionic in nature and is determined by the concentration of impurities 
(alkali-metal ions) which are always present as trace constituents. Two more examples are doped semiconductors with dopant concentration below critical and dielectriclike semimetals.

As shown in Sec. V.B, the modification of the transverse magnetic reflection coefficient at zero frequency in accordance with Eq. (53) is in contradiction with the data of the experiment on the optically modulated $\mathrm{Ca}$ simir forces (Chen et al., 2007a, 2007b). This is in line with the fact that, being the particular case of Eq. (40), the modified reflection coefficient (53) violates the applicability conditions of the Lifshitz theory.

\section{HOW TO COMPARE THEORY AND EXPERIMENT}

\section{A. Modeling of the optical properties of real materials}

\section{Kramers-Kronig relations}

To compare theory and experiment one first needs reliable theoretical results. These results can be obtained using the Lifshitz formula (3) with an appropriate dielectric permittivity for the plate material along the imaginary frequency axis. In general, it can be obtained from the imaginary part of complex dielectric permittivity $\varepsilon(\omega)=\varepsilon^{\prime}(\omega)+i \varepsilon^{\prime \prime}(\omega)$ and the Kramers-Kronig relations. The imaginary part of the permittivity is $\varepsilon^{\prime \prime}(\omega)$ $=2 n^{\prime}(\omega) n^{\prime \prime}(\omega)$, where $n^{\prime}(\omega)$ and $n^{\prime \prime}(\omega)$ are the real and imaginary parts of the complex index of refraction. The tabulated optical data for $n^{\prime}(\omega)$ and $n^{\prime \prime}(\omega)$ for the different materials can be found in Palik (1985). It should be noted that the form of Kramers-Kronig relations is different depending on the analytic properties of the dielectric permittivity under consideration. If $\varepsilon(\omega)$ is a regular function at $\omega=0$ [the case of dielectrics with dielectric permittivity given by Eq. (44)], the KramersKronig relations take the simplest form (Jackson, 1999),

$$
\begin{aligned}
& \varepsilon^{\prime}(\omega)=1+\frac{1}{\pi} \mathrm{P} \int_{-\infty}^{\infty} \frac{\varepsilon^{\prime \prime}(\xi)}{\xi-\omega} d \xi, \\
& \varepsilon^{\prime \prime}(\omega)=-\frac{1}{\pi} \mathrm{P} \int_{-\infty}^{\infty} \frac{\varepsilon^{\prime}(\xi)}{\xi-\omega} d \xi,
\end{aligned}
$$

where the integrals are understood as a principal value. The third dispersion relation, expressing the dielectric permittivity along the imaginary frequency axis, in this case is

$$
\varepsilon(i \xi)=1+\frac{2}{\pi} \int_{0}^{\infty} \frac{\omega \varepsilon^{\prime \prime}(\omega)}{\xi^{2}+\omega^{2}} d \omega
$$

The optical data for dielectrics are available in a wide frequency range $\left[\omega_{\min }, \omega_{\max }\right]$ (Palik, 1985). Near $\omega_{\min }$, $\varepsilon^{\prime \prime}(\omega)$ is almost zero. Bearing in mind that $\omega_{\min }$ is usually below all the relevant resonances of the respective dielectric medium, it is possible to extrapolate $\varepsilon^{\prime \prime}(\omega)$ as zero in the region $0 \leqslant \omega \leqslant \omega_{\min }$. Regarding $\omega_{\max }$, it is usually sufficiently high that no extrapolation to region $\omega$ $>\omega_{\max }$ is required. There are also many approximate analytic expressions for the dielectric permittivity (44) [see, e.g., Hough and White (1980), Bergström (1997), and Parsegian (2005)] suggested for the calculation of the Hamaker constant at short separation distances in the region of the nonretarded van der Waals force. These expressions can also be used for calculations of the retarded Casimir forces using the Lifshitz formula (3) (Parsegian, 2005).

The description of the dielectric properties of metals is much more complicated. In calculations of the zerotemperature Casimir energy and pressure [Eqs. (12) and (13)] the plasma model dielectric permittivity (32) is often used, following Lifshitz (1956). This model is, however, applicable only at separation distances larger than the plasma wavelength, as it does not take into account the absorption bands due to the core electrons. To address this problem, there are two approaches suggested in the literature. In the first approach the tabulated optical data for the complex index of refraction of the metal under consideration are used to obtain the imaginary part of the dielectric permittivity. The latter is extrapolated to the region of low frequencies with the help of the Drude dielectric function (Lamoreaux, 1999; Klimchitskaya et al., 2000; Lambrecht and Reynaud, 2000). In the second approach the core electrons are described by a set of oscillators with nonzero oscillator frequencies [similar to Eq. (44) (Jackson, 1999)] but the free conduction electrons are described using the plasma model (Decca et al., 2007b; Geyer et al. 2007; Klimchitskaya et al., 2007a; Mostepanenko and Geyer, 2008). In this case we obtain the so-called generalized plasmalike dielectric permittivity,

$$
\varepsilon_{\mathrm{gp}}(\omega)=\varepsilon_{c}(\omega)-\frac{\omega_{p}^{2}}{\omega^{2}}=1-\frac{\omega_{p}^{2}}{\omega^{2}}+\sum_{j} \frac{g_{j}}{\omega_{j}^{2}-\omega^{2}-i \gamma_{j} \omega} .
$$

The values of the oscillator parameters of core electrons are found from the tabulated optical data for the complex index of refraction (Palik, 1985). Since the Casimir effect is a broadband phenomenon, it is of utmost importance to use as exact data as possible, determined over a wide frequency range in the interband absorption region $\omega>2.5 \mathrm{eV}$. Presently such data (and also data for lower frequencies) can be obtained using an ellipsometer. Thus, Svetovoy et al. (2008) found precise optical properties of several $\mathrm{Au}$ films in the frequency region from 0.73 to $8.86 \mathrm{eV}$ using a vacuum ultraviolet ellipsometer (and from 0.038 to $0.65 \mathrm{eV}$ using an infrared variable-angle spectroscopic ellipsometer).

In both approaches the dielectric permittivity along the imaginary frequency axis can be obtained by means of the Kramers-Kronig relations. Regardless of which approach is used, the dielectric permittivity of a metal is not regular at $\omega=0$ but has a pole. This changes the form of the Kramers-Kronig relations (56). If $\varepsilon(\omega)$ has a simple pole at $\omega=0, \varepsilon(\omega) \approx 4 \pi i \sigma_{0} / \omega$, the first two Kramers-Kronig relations are (Landau et al., 1984)

$$
\varepsilon^{\prime}(\omega)=1+\frac{1}{\pi} \mathrm{P} \int_{-\infty}^{\infty} \frac{\varepsilon^{\prime \prime}(\xi)}{\xi-\omega} d \xi
$$




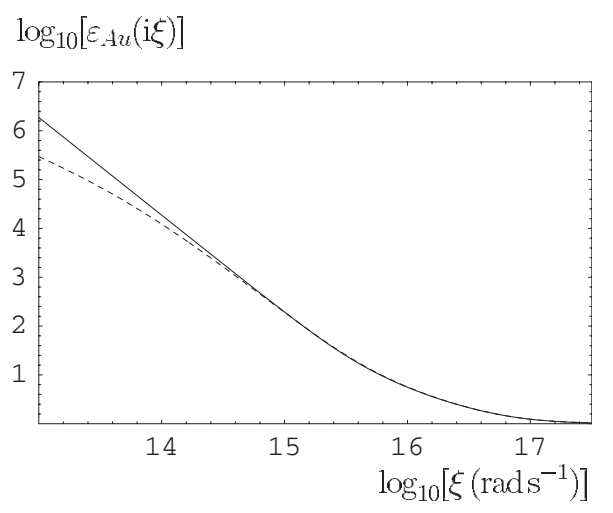

FIG. 1. The dielectric permittivity of Au along the imaginary frequency axis. The solid line is obtained using the generalized plasmalike model taking into account the optical data related to the core electrons. The dashed line is obtained using the optical data extrapolated to low frequencies by the Drude model.

$$
\varepsilon^{\prime \prime}(\omega)=-\frac{1}{\pi} \mathrm{P} \int_{-\infty}^{\infty} \frac{\varepsilon^{\prime}(\xi)}{\xi-\omega} d \xi+\frac{4 \pi \sigma_{0}}{\omega} .
$$

The third dispersion relation in this case is given by Eq. (57), i.e., remains the same as for a dielectric permittivity regular at $\omega=0$.

We now consider the generalized plasmalike dielectric permittivity (58) having a second-order pole at zero frequency [i.e., an asymptotic behavior $\varepsilon(\omega) \approx-\omega_{p}^{2} / \omega^{2}$ when $\omega \rightarrow 0]$. The standard derivation (Landau et al., 1984; Jackson, 1999), when applied to this case, leads to one more form of the Kramers-Kronig relations (Klimchitskaya et al., 2007a),

$$
\begin{aligned}
& \varepsilon^{\prime}(\omega)=1+\frac{1}{\pi} \mathrm{P} \int_{-\infty}^{\infty} \frac{\varepsilon^{\prime \prime}(\xi)}{\xi-\omega} d \xi-\frac{\omega_{p}^{2}}{\omega^{2}} \\
& \varepsilon^{\prime \prime}(\omega)=-\frac{1}{\pi} \mathrm{P} \int_{-\infty}^{\infty} \frac{\varepsilon^{\prime}(\xi)+\frac{\omega_{p}^{2}}{\xi^{2}}}{\xi-\omega} d \xi .
\end{aligned}
$$

In this case the third Kramers-Kronig relation (57) is also replaced with (Klimchitskaya et al., 2007a),

$$
\varepsilon(i \xi)=1+\frac{2}{\pi} \int_{0}^{\infty} \frac{\omega \varepsilon^{\prime \prime}(\omega)}{\xi^{2}+\omega^{2}} d \omega+\frac{\omega_{p}^{2}}{\xi^{2}},
$$

i.e., it acquires an additional term.

\section{Calculation for $\mathrm{Au}$}

We now discuss the application of the above two approaches to calculations of the dielectric permittivity along the imaginary frequency axis for $\mathrm{Au}$, a metal widely used in the recent Casimir force measurements (see Secs. IV-VI). As discussed, both approaches use the tabulated optical data for the complex index of refraction (Palik, 1985). The calculation results are presented in Fig. 1. The dashed line in Fig. 1 is obtained using the Kramers-Kronig relation (57) with $\varepsilon^{\prime \prime}(\omega)$ given by
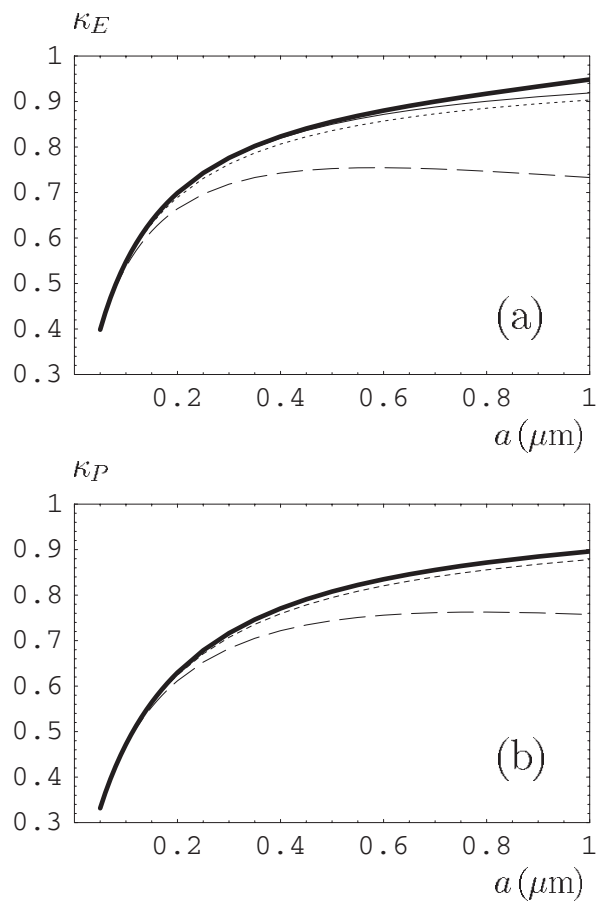

FIG. 2. Correction factors to (a) the Casimir energy per unit area and (b) the pressure vs separation between Au plates. Bold and fine solid lines are computed using the generalized plasmalike model at $T=300 \mathrm{~K}$ and 0 , respectively. Long- and short-dashed lines are calculated using the extrapolation of optical data by the Drude model at $T=300 \mathrm{~K}$ and 0 , respectively.

the complete tabulated optical data extrapolated to low frequencies by the imaginary part of the Drude dielectric function with $\omega_{p}=9.0 \mathrm{eV}$ and $\gamma=0.035 \mathrm{eV}$ (Klimchitskaya et al., 2000; Lambrecht and Reynaud, 2000). The solid line in Fig. 1 is obtained using the KramersKronig relation (61) adapted for the generalized plasmalike model with $\varepsilon^{\prime \prime}(\omega)$ describing the contribution of core electrons. In this case $\varepsilon^{\prime \prime}(\omega)$ is given by the tabulated data with the contribution of free conduction electrons subtracted (Decca et al., 2007b) using $\omega_{p}=9.0 \mathrm{eV}$. Note that for frequencies $\xi<10^{16} \mathrm{rad} / \mathrm{s}$ the simple analytic six-oscillator model can also be used for the description of core electrons (Decca et al., 2007b; Mostepanenko and Geyer, 2008). As is seen in Fig. 1, the computational results for $\varepsilon(i \xi)$ using the two approaches coincide at $\xi$ $\geqslant 10^{15} \mathrm{rad} / \mathrm{s}$ but have different behavior at lower frequencies.

In Fig. 2 we present the computational results for the correction factors to the Casimir energy per unit (a) area and (b) pressure obtained using the dielectric permittivities presented in Fig. 1 by the solid and dashed lines. These correction factors are defined as

$$
\kappa_{E}(a, T)=\frac{\mathcal{F}(a, T)}{E_{0}(a)}, \quad \kappa_{P}(a, T)=\frac{P(a, T)}{P_{0}(a)},
$$

where $E_{0}(a)$ and $P_{0}(a)$ are the Casimir energy and pressure between ideal metal planes defined in Eq. (2). The solid lines represent the computational results using the generalized plasmalike model [bold lines are obtained 
using Eqs. (3) and (10) at $T=300 \mathrm{~K}$ whereas fine lines using the zero-temperature Eqs. (12) and (13); in the case of the Casimir pressure bold and fine solid lines practially coincide at all $a \leqslant 1 \mu \mathrm{m}]$. The dashed lines correspond to computations using the tabulated optical data extrapolated by the Drude model [the long-dashed lines are obtained using Eqs. (3) and (10) at $T=300 \mathrm{~K}$ and the short-dashed lines using the zero-temperature Lifshitz formulas (12) and (13) with the dielectric permittivity at room temperature]. As is seen in Figs. 2(a) and 2(b), there are large deviations between the zerotemperature short-dashed and $T=300 \mathrm{~K}$ long-dashed lines at all separation distances above $100 \mathrm{~nm}$. This means that the zero-temperature formulas (12) and (13) with substituted room-temperature values of the dielectric permittivity accounting for the relaxation processes of conduction electrons can only be approximately used at short separation distances, i.e., in the region of the van der Waals forces and in the beginning of the transition region to the Casimir forces (Parsegian, 2005). At larger separation distances Eqs. (12) and (13) do not reproduce the values of the Casimir free energy. Using this model of the dielectric permittivity, all calculations at separations above $100 \mathrm{~nm}$ at room temperature should be done with the thermal Lifshitz formulas (3) and (10) for the Casimir free energy per unit area and pressure. We emphasize, however, that the theoretical results obtained in this way are excluded by an experiment at a high confidence level (see Sec. IV.B).

The preceding discussion sheds light on the problem of sample-to-sample dependence of the optical data. Actually, this dependence is mostly determined by the relaxation processes of free conduction electrons at infrared and optical frequencies [e.g., due to different characteristic sizes of grains in thin films (Sotelo et al., 2003)]. Uncertainty of the Casimir pressure due to the variation of optical properties was investigated by Pirozhenko et al. (2006) and Svetovoy et al. (2008) using the zero-temperature Lifshitz formula (13). For this purpose both the real and imaginary parts of the dielectric permittivity of $\mathrm{Au}$, as given by the optical data in the infrared region collected by others, were approximated by the real and imaginary parts of the Drude permittivity with an additional polarization term. It is easily seen that all sets of the Drude parameters obtained are excluded by the experiment on the measurement of the Casimir pressure [see the general method of the verification of hypotheses, as applied to this case, in Sec. III.C.2 and Fig. 11(b) in Sec. IV.B]. In contrast, the generalized plasmalike dielectric permittivity is based on the characteristics of materials determined by the structure of a crystal cell ( $\omega_{p}$ is one such factor). These characteristics are not sensitive to sample-to-sample variations of the optical properties of the deposited polycrystal films. Large variations in the values of $\omega_{p}$ obtained by Pirozhenko et al. (2006) for different Au samples can be explained by the fact that the authors used the Drude model with frequency-independent relaxation parameter, whereas at high frequencies the relaxation parameter of conduction electrons depends on the frequency (Lifshitz et al., 1973). As discussed, the drift current of conduction electrons is not compatible with the Lifshitz theory of dispersion forces (Parsegian, 2005; Geyer et al., 2007; Mostepanenko and Geyer, 2008). Because of this, sample-to-sample variations of scattering processes connected with this current should be disregarded in the theory of the Casimir force.

\section{Problem of spatial dispersion}

Another problematic issue is connected with the role of spatial dispersion. In particular, it is well known that in the frequency region of the anomalous skin effect metals cannot be described by the dielectric permittivity depending only on the frequency (Lifshitz et al., 1973; Lifshitz and Pitaevskii, 1981). The Lifshitz formulas (3), (6), (10), (12), and (13), however, are derived only for materials that can be described by $\varepsilon(\omega)$. The application range of the Lifshitz theory can be widened using the Leontovich surface impedance boundary condition (Landau et al., 1984). Kats (1977) pioneered the application of this approach to describe metals in the region of the anomalous skin effect at zero temperature. The Leontovich approach makes it possible to take into account real material properties without considering the interior of the plates. In this approach the standard continuity boundary conditions applicable to interfaces between spatially local media are not followed. The Leontovich impedance approach now appears interesting for the description of the thermal Casimir force. It was suggested (Bezerra et al., 2001; Geyer et al., 2003) to extrapolate the impedance for the frequency region around the characteristic frequency $\omega_{c}=c /(2 a)$ to all frequencies. For separation distances related to infrared optics, some, however, have used the form of impedance for the normal (Torgerson and Lamoreaux, 2004) or the anomalous (Svetovoy and Lokhanin, 2003) skin effect. In these latter cases the theoretical results turn out to be in violation of the Nernst heat theorem and in contradiction with experiment (Geyer et al., 2003, 2004; Bezerra et al., 2007).

There is another approach to account for spatial dispersion by the substitution of the dielectric permittivity depending on both the frequency and wave vector, $\varepsilon(\omega, \boldsymbol{k})$, into the usual Lifshitz formulas (3) and (8) or (12) and (13) with some modified reflection coefficients. This approach is, however, unjustified. The review by Barash and Ginzburg (1975) contains a few references to incorrect results obtained by others using such substitutions. In the last few years, more papers have appeared (Esquivel et al., 2003; Esquivel and Svetovoy, 2004; Contreras-Reyes and Mochán, 2005; Sernelius, 2005; Svetovoy and Esquivel, 2005) employing the same approach in an attempt to solve the problem resulting from using the Drude dielectric permittivity in the Lifshitz formula. It was demonstrated (Klimchitskaya and Mostepanenko, 2007) that these attempts are not warranted because the modified reflection coefficients are derived using phenomenological (additional) boundary conditions and dielectric permittivities $\varepsilon(\omega, \boldsymbol{k})$ which are 
ill-defined quantities in the presence of a gap between the plates as it violates the assumption of translational invariance in the $z$ direction. In the presence of spatial dispersion, the boundary conditions following from the Maxwell equations differ from the standard continuity boundary conditions due to the inclusion of the induced charge and current densities (Agranovich and Ginzburg, 1984; Ginzburg, 1985). It was also shown that in the presence of the boundary surface the dielectric permittivities depending on $\omega, \boldsymbol{k}$ can be approximately used for the description of the remainder of the medium except for a layer adjacent to the boundary surface (Agranovich and Ginzburg, 1984). However, it is unlikely that this phenomenological approach would be applicable for the calculation of the Casimir force between metallic surfaces where the boundary effects are of prime importance. The generalization of the Lifshitz formula in terms of the functional determinants and scattering matrices [see Eq. (17)] opens opportunities to include the effects of spatial dispersion. For the configuration of two plane parallel plates the matrix elements of the operator $\mathcal{G}_{\xi, A B}^{(0)}$ reduce to a factor $\exp (-q a)$ and the same holds for $\mathcal{G}_{\xi, B A}^{(0)}$ (Lambrecht et al., 2006). To describe the plates including the spatial dispersion, one should find the respective $T$ matrices of the operators $\mathcal{T}^{A}$ and $\mathcal{T}^{B}$. In principle, this could be done by solving Maxwell's equations for a given Casimir configuration made of a nonlocally responding material without using the dielectric permittivity depending on $\omega$ and $\boldsymbol{k}$. This problem awaits its resolution.

\section{B. Corrections to the Casimir force due to the imperfect geometry of interacting bodies}

\section{Surface roughness}

The problem of roughness corrections to dispersion forces has long attracted the attention of researchers [see, e.g., Maradudin and Mazur (1980), Mazur and Maradudin (1981), Derjaguin et al. (1987), and Rabinovich and Churaev (1989), where roughness corrections to the nonretarded van der Waals force were investigated]. These corrections can be calculated using perturbation theory and Green's functions (Balian and Duplantier, 1977, 1978), functional integration (Golestanian and Kardar, 1997, 1998; Emig et al., 2001, 2003), or by the phenomenological methods of pairwise summation or geometrical averaging (Bordag et al., 1994, 1995a, 1995b; Klimchitskaya et al., 1999). In doing so some small parameters should be introduced which characterize the deviation from basic geometry.

Consider two plates whose surfaces possess small deviations from the plane geometry. The surfaces of these plates can be described by

$$
z_{1}^{(s)}=A_{1} f_{1}\left(x_{1}, y_{1}\right), \quad z_{2}^{(s)}=a+A_{2} f_{2}\left(x_{2}, y_{2}\right),
$$

where $a$ is the mean value of the separation distance between the plates. The values of the amplitudes are chosen in such a way that $\max \left|f_{i}\left(x_{i}, y_{i}\right)\right|=1$. The averaging of Eqs. (63) over the total area of the plates with appropriately chosen origin on the $z$ axis leads to

$$
\begin{aligned}
& \left\langle z_{1}^{(s)}\right\rangle=A_{1}\left\langle f_{1}\left(x_{1}, y_{1}\right)\right\rangle=0, \\
& \left\langle z_{2}^{(s)}\right\rangle=a+A_{2}\left\langle f_{2}\left(x_{2}, y_{2}\right)\right\rangle=a .
\end{aligned}
$$

As mentioned, we consider small deviations from the basic geometry, i.e., $A_{i} \ll a$.

The topography of the surfaces (63) can be obtained experimentally as an atomic force microscope (AFM) image of a rather large area (see, e.g., Fig. 8 in Sec. IV.A). AFM images of the surfaces of test bodies used in the experiments described in Secs. IV.A, IV.B, V, and VII show that the roughness is represented by stochastically distributed distortions of different heights with a typical lateral correlation length of about $200-400 \mathrm{~nm}$. If the correlation effects can be neglected (below we discuss why they are small), the surface topography is approximately characterized by a discrete set of pairs $\left(v_{i}^{(1,2)}, h_{i}^{(1,2)}\right)$, where $v_{i}^{(1,2)}$ is the fraction of the surface area with height $h_{i}^{(1,2)}$ on the surface 1 or 2, respectively. These data allow one to determine the zero-roughness levels $H_{0}^{(1,2)}$ relative to which the mean values of the functions $f_{1,2}$ in Eq. (63) are equal to zero,

$$
\sum_{i=1}^{K^{(k)}}\left(H_{0}^{(k)}-h_{i}^{(k)}\right) v_{i}^{(k)}=0, \quad k=1,2 .
$$

Note that the separation distances between the test bodies in the Casimir force measurements are measured just between these zero-roughness levels.

The Casimir pressure between the two ideal metal plates with rough surfaces [Eq. (63)] at zero temperature can be represented in the form

$$
P(a)=-\left(\pi^{2} \hbar c / 240 a^{4}\right) \kappa_{P}^{(r)},
$$

where $\kappa_{P}^{(r)}$ is the correction factor due to surface roughness. In the framework of the pairwise summation method, which is based on the additive summation of the Casimir-Polder interatomic potentials with the subsequent normalization of the obtained interaction constant, this correction factor calculated up to the fourth perturbation order in relative amplitudes $A_{i} / a$ is (Bordag et al., 1995a)

$$
\begin{aligned}
\kappa_{P}^{(r)}= & 1+\frac{10}{a^{2}}\left(\left\langle f_{1}^{2}\right\rangle A_{1}^{2}-2\left\langle f_{1} f_{2}\right\rangle A_{1} A_{2}+\left\langle f_{2}^{2}\right\rangle A_{2}^{2}\right) \\
& +\frac{20}{a^{3}}\left(\left\langle f_{1}^{3}\right\rangle A_{1}^{3}-3\left\langle f_{1}^{2} f_{2}\right\rangle A_{1}^{2} A_{2}+3\left\langle f_{1} f_{2}^{2}\right\rangle A_{1} A_{2}^{2}\right. \\
& \left.-\left\langle f_{2}^{3}\right\rangle A_{2}^{3}\right)+\frac{35}{a^{4}}\left(\left\langle f_{1}^{4}\right\rangle A_{1}^{4}-4\left\langle f_{1}^{3} f_{2}\right\rangle A_{1}^{3} A_{2}\right. \\
& \left.+6\left\langle f_{1}^{2} f_{2}^{2}\right\rangle A_{1}^{2} A_{2}^{2}-4\left\langle f_{1} f_{2}^{3}\right\rangle A_{1} A_{2}^{3}+\left\langle f_{2}^{4}\right\rangle A_{2}^{4}\right) .
\end{aligned}
$$

The mixed terms in Eq. (67) represent interference behavior.

For example, in the case of two plane plates with a small angle $\alpha$ between them, it follows from Eq. (67) that 


$$
\kappa_{P}^{(r)}=1+\frac{10}{3}(\alpha L / a)^{2}+7(\alpha L / a)^{4},
$$

where $2 L$ is the characteristic length of the plate. As is easily seen from Eq. (68), for plates with $L \approx 1 \mathrm{~mm}$ at a separation $a \approx 500 \mathrm{~nm}$ the correction due to nonparallelism of the plates is less than $1 \%$ if $\alpha$ does not exceed $2.7 \times 10^{-5} \mathrm{rad}$. This is the reason why the experiments with two plane plates demand a high degree of parallelity. Note also that the small average separations needed for measurements cannot be achieved if the plates are tilted, as they might make contact at the edges. For the above example the contact of the plate edges occurs at a tilt angle of $1.72^{\prime}=5 \times 10^{-4} \mathrm{rad}$. The correction factor $\kappa_{E}^{(r)}$ to the Casimir energy between the two ideal metal plates,

$$
E(a)=-\left(\pi^{2} \hbar c / 720 a^{3}\right) \kappa_{E}^{(r)},
$$

is connected to Eq. (67) by

$$
\kappa_{P}^{(r)}=\kappa_{E}^{(r)}-\frac{1}{3} d \kappa_{E}^{(r)} / d a .
$$

In practical computations it is often convenient not to consider the complete roughness topography of surfaces (63) but to describe the roughness as a stochastic process by its rms variance,

$$
\delta_{\mathrm{st}, k}^{2}=\sum_{i=1}^{K^{(k)}}\left(H_{0}^{(k)}-h_{i}^{(k)}\right)^{2} v_{i}^{(k)} .
$$

If roughness can be characterized by the rms variance, the correction factor to the Casimir pressure between the two parallel plates takes a simpler form (Bordag et al., 1995b)

$$
\kappa_{P}^{(r)}=1+\frac{10}{a^{2}}\left(\delta_{\mathrm{st}, 1}^{2}+\delta_{\mathrm{st}, 2}^{2}\right)+\frac{105}{a^{4}}\left(\delta_{\mathrm{st}, 1}^{2}+\delta_{\mathrm{st}, 2}^{2}\right)^{2} .
$$

For specially prepared surfaces in modern Casimir experiments, the roughness corrections calculated using Eqs. (67) and (72) usually lead to the same result. The correction factor to the Casimir energy due to stochastic roughness takes the form (Bordag et al., 1995b)

$$
\kappa_{E}^{(r)}=1+\frac{6}{a^{2}}\left(\delta_{\mathrm{st}, 1}^{2}+\delta_{\mathrm{st}, 2}^{2}\right)+\frac{45}{a^{4}}\left(\delta_{\mathrm{st}, 1}^{2}+\delta_{\mathrm{st}, 2}^{2}\right)^{2} .
$$

For the configuration of a sphere (spherical lens) above a plate, which is preferred from an experimental point of view, the investigation of roughness corrections to the Casimir force in the framework of the pairwise summation method was performed by Klimchitskaya and Pavlov (1996). It was demonstrated that if the characteristic lateral scales of surface roughness on a plate $\Lambda_{p}$ and on a sphere $\Lambda_{s}$ are rather small, $\Lambda_{p}, \Lambda_{s} \ll \sqrt{a R}$, the correction factor to the Casimir force between a sphere and a plate made of ideal metals is the same as for the Casimir energy between the two ideal metal plates, i.e., is equal to $\kappa_{E}^{(r)}$.

If the above inequalities are not fulfilled (e.g., there are significant deviations of the lens surface from a spherical shape), an additional term of the order $\sim 1 / a$ results in the correction factor. That is why it is neces- sary to check carefully that the curvature radius of the sphere is constant. The role of roughness in atom-wall interaction was investigated by Bezerra, Klimchitskaya, and Romero (2000).

The dependence of the Casimir force between metallic test bodies on the penetration depth of the electromagnetic oscillations into the metal (i.e., on the skin depth) was first demonstrated by Mohideen and Roy (1998). This raised the question of how to take into account both the conductivity and roughness corrections. The simplest approach is a multiplicative one: to calculate the Casimir free energy (3) or the Casimir pressure (10) using real material properties, including the role of the skin depth, and multiply the obtained results by the correction factor for ideal metal plates, $\kappa_{E}^{(r)}$ or $\kappa_{P}^{(r)}$, respectively [see, e.g., Mohideen and Roy (1998) and Chan et al. (2001a)]. This approach corresponds to the addition of the different errors if they are small. Thus, it is not applicable when at least one of the corrections is large. At short separation distances $a$, where the conductivity corrections are not small and strongly depend on $a$ [see Figs. 2(a) and 2(b)], more sophisticated methods are needed.

The nonmultiplicative approach of the geometrical averaging was elaborated by Klimchitskaya et al. (1999). According to this method, the Casimir pressure between the two rough plates made of real materials can be calculated as

$$
\begin{aligned}
P^{(r)}(a, T)= & \sum_{i=1}^{K^{(1)}} \sum_{j=1}^{(2)} v_{i}^{(1)} v_{j}^{(2)} \\
& \times P\left(a+H_{0}^{(1)}+H_{0}^{(2)}-h_{i}^{(1)}-h_{j}^{(2)}, T\right) .
\end{aligned}
$$

Here $P(z, T)$ is calculated using Eq. (8) including real material properties. Note that Eq. (74) is not reduced to a simple multiplication of the correction factors due to finite conductivity and surface roughness but takes into account their combined (nonmultiplicative) effect as well.

In a similar way, the Casimir free energy for two rough plates can be calculated as

$$
\begin{aligned}
\mathcal{F}^{(r)}(a, T)= & \sum_{i=1} \sum_{j=1}^{(1)} v_{i}^{(1)} v_{j}^{(2)} \\
& \times \mathcal{F}\left(a+H_{0}^{(1)}+H_{0}^{(2)}-h_{i}^{(1)}-h_{j}^{(2)}, T\right),
\end{aligned}
$$

where $\mathcal{F}(z, T)$ is given in Eq. (3). The method of geometrical averaging is widely used when comparing theoretical and experimental results in experiments on the measurements of the Casimir force [see, e.g., Klimchitskaya et al. (1999), Decca, Fischbach, et al. (2003), Decca, López, et al. (2003), Chen, Klimchitskaya, Mohideen, and Mostepanenko (2004), Decca, López, Fischbach, et al. (2005), Decca et al. (2007a, 2007b), Lisanti et al. (2005), and Munday and Capasso (2007)].

Equations (74) and (75) are based, however, on the proximity force approximation and do not take into account the diffractionlike correlation effects. In firstorder perturbation theory, these effects have been inves- 
tigated for ideal metal plates covered with periodic roughness (Emig et al., 2001, 2003) and for metals described by the plasma model covered with stochastic roughness (Genet, Lambrecht, Maia Neto, and Reynaud, 2003; Maia Neto et al., 2005). As demonstrated, the correlation effects noticeably contribute to the roughness correction only at relatively large separations of order or much larger than the roughness period $\Lambda$ (or the correlation length) [see also van Zwol et al. (2007)]. The special analysis (Chen, Klimchitskaya, Mohideen, and Mostepanenko, 2004; Decca, López, Fischbach, et al., 2005), based on the results by Emig et al. (2001, 2003) showed that at separation distances $a$ a few times smaller than $\Lambda$ the role of correlation effects is negligibly small (about $0.03-0.04 \%$ change in the magnitude of the roughness correction for the roughness topography of test bodies used in the experiments). With an increase of separation distance the correlation effects become more important, but at such large separations the complete roughness correction including the correlation effects is negligibly small. Thus, an accurate account of surface roughness corrections would need more sophisticated methods beyond the pairwise summation approach or geometrical averaging for test bodies with roughness having a small correlation length. In this case there would be large correlation effects even at relatively short separations where the role of roughness is significant. The situation may become even more complicated if the roughness amplitudes are not small and one has to go beyond the lowest-order perturbation theory. With respect to the roughness corrections, to date, however, there are no measurements of the Casimir force which fall into this category. The first experimental evidence for the effects beyond the proximity force approximation was obtained by Chan et al. (2008) measuring the Casimir interaction between a Au sphere and a Si plate with deep rectangular trenches. This experiment is discussed in Sec. V.D.

There is one more effect connected with surface roughness which should be accounted for in Casimir force measurements. It is conceivable that the spatial variations of the surface potentials due to grains of the polycrystalline metal film (the so-called patch potentials) simulate the Casimir force. Speake and Trenkel (2003) obtained general expressions for the electrostatic free energy and pressure in the configuration of two parallel plates, which result from random variations of patch potentials. These expressions can be used to analyze the role of patch effects in any performed experiment. In particular, it was shown (Chen, Klimchitskaya, Mohideen, and Mostepanenko, 2004; Decca, López, Fischbach, et al., 2005) that in the most precise experiments measuring the Casimir force between metallic test bodies (see Secs. IV.A and IV.B) the patch effects are negligibly small even at the shortest separation distances.

\section{Finite size and thickness}

The last point to be considered in this section is the influence of the finite size and a thickness of interacting bodies on the calculational results. In fact, Eqs. (3) and (10) for the Casimir free energy per unit area and pressure are obtained for plates of infinite area. Using the proximity force approximation, we obtain from Eq. (3) the Casimir force between a sphere of radius $R \gg a$ above an infinitely large plate. In experimental configurations, however, the plate always has a finite size, and, instead of a sphere, a spherical lens of some thickness $H<R$ may be used. It should be noted that recently rigorous methods based on quantum field theory first principles have been developed which allow one to calculate the scalar and electromagnetic Casimir forces between arbitrarily shaped compact objects [see, e.g., Gies and Klingmüller (2006a, 2006b, 2006c) and Emig et al. $(2007,2008)]$. However, the application of these methods for calculation of small corrections due to the finite sizes of the test bodies in experimental configurations, where the separation distances are rather small, still remains a problem (especially in the electromagnetic case). For this reason, finite size corrections still need to be estimated using the simple method of pairwise summation. For a spherical lens of radius $R$ and thickness $H$ placed at a distance $a$ above a disk of radius $L$ the finite size correction factor to the Casimir force is given by (Bezerra et al., 1997)

$$
\kappa_{\mathrm{fs}}=1-\left(a^{3} / R^{3}\right)(1-T)^{-3} .
$$

Here $T \equiv \max \left[R / \sqrt{R^{2}+L^{2}},(R-H) / R\right]$. Using Eq. (76) one can estimate that in all experiments performed to date (see Secs. IV and V) the correction due to the finite size of the test bodies is negligibly small. Thus, it is reasonable to compare experimental results with the theoretical computations using Eq. (3) or (10).

The finite thickness of plates can be taken into account by the replacement of reflection coefficients (6) with those obtained for multilayered structures (Zhou and Spruch, 1995; Klimchitskaya et al., 2000). For metal plates of larger thickness than the skin depth in the frequency region of infrared optics $\left(\delta_{0}=c / \omega_{p} \approx 22 \mathrm{~nm}\right.$ for $\mathrm{Au})$ the calculational results are the same as those for semispaces. Thus, for typical metal films of about $100 \mathrm{~nm}$ thickness deposited on the surfaces of test bodies in different experiments, the Lifshitz formulas for semispaces can be reliably used (Klimchitskaya et al., 2000). As an example, if in one case we have two Au semispaces at a separation of $200 \mathrm{~nm}$ and in the other a $\mathrm{Au}$ semispace at the same separation from a 100-nm-thick Au film made on a Si semispace substrate, the relative difference in the Casimir energy is less than $0.01 \%$. Recent experiments on the measurement of the Casimir force between a metallic sphere and semiconductor (Si) plate (Chen et al., 2005, 2007a, 2007b; Chen, Klimchitskaya, et al., 2006; Chen, Mohideen, et al., 2006), have created an interest in the role of the thickness of the Si plate (Duraffourg and Andreucci, 2006; Lambrecht et al., 2007; Pirozhenko and Lambrecht, 2008a). In these papers the Lifshitz formula at zero temperature is used in the computations and, in the latter two, large separations up to $1 \mathrm{~mm}$ were considered. The depen- 


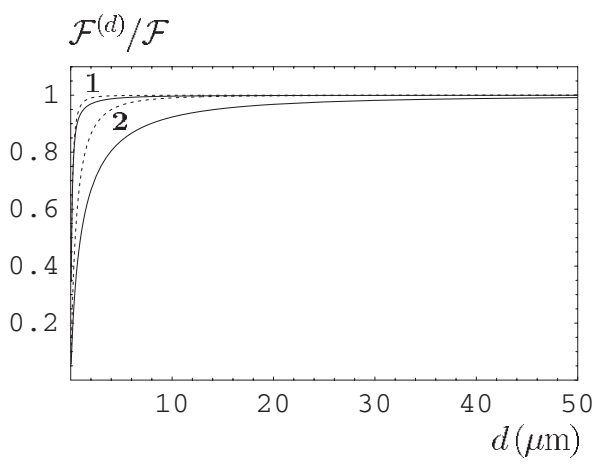

FIG. 3. The ratio of the Casimir free energy for a Si plate of thickness $d$ at separations $a=1$ (lines labeled 1) and $5 \mu \mathrm{m}$ (lines labeled 2) from $\mathrm{Au}$ semispace to the free energy in the configuration of $\mathrm{Si}$ and $\mathrm{Au}$ semispaces at the same separations. Solid lines are computed at $T=300 \mathrm{~K}$. Dashed lines are computed at $T=0$.

dences of the Casimir force on the plate thickness $d$ and the doping concentration are investigated. The results obtained are, however, not applicable at large separation distances at the laboratory temperature $T=300 \mathrm{~K}$. For example, we plot in Fig. 3 the ratio of the Casimir free energy for the configuration of a Si plate of thickness $d$ at a separation $a$ from a Au semispace to the free energy in the configuration of $\mathrm{Si}$ and Au semispaces, as a function of Si plate thickness $d$. Solid lines 1 and 2 are computed at $T=300 \mathrm{~K}$ at $a=1$ and $5 \mu \mathrm{m}$, respectively. Dashed lines 1 and 2 are computed at $T=0$ at the same separations. As is seen in the figure, at $a=5 \mu \mathrm{m}$ the deviation between the solid and dashed lines reaches $20 \%$. Thus, the role of finite thickness of the plate at separations above $1 \mu \mathrm{m}$ should be investigated using the Lifshitz formula at nonzero temperature.

\section{Quantitative comparison between experiment and theory in force-distance measurements}

\section{Experimental errors and precision}

When comparing experimental data from Casimir force experiments with theoretical predictions, the important question on how to quantitatively characterize the measure of agreement between them has to be addressed. Previously the concept of the root-mean-square deviation between theory and experiment has been used to quantify the precision of measurements and the agreement with theory [see, e.g., early experiments by Bordag et al. (2001) and also some later experiments (Bressi et al., 2002; Decca, Fischbach, et al., 2003; Chen, Klimchitskaya, Mohideen, and Mostepanenko, 2004)]. It is well known, however, that this method is not appropriate for strongly nonlinear quantities, such as the Casimir force, which changes rapidly with separation distance (Rabinovich, 2000). It was shown (Ederth, 2000) that the calculation of the root-mean-square deviation between experiment and theory leads in this case to different results when applied in different measurements ranges but no better method was suggested. Later, a rig- orous approach to the comparison of experiment and theory in Casimir force measurements was presented (Decca, López, Fischbach, et al., 2005; Chen, Mohideen, et al., 2006; Klimchitskaya, Chen, et al., 2006) using statistical methods.

The first step in the application of this approach is to characterize how precise the experimental data are without any relation to the theory. To do this, the absolute and relative total experimental errors should be calculated as a combination of systematic and random errors. We use in the error analysis the notation $\Pi^{\operatorname{expt}}(a)$ for the measured quantity, which is either the Casimir pressure $P^{\operatorname{expt}}(a)$ in the configuration of two parallel plates or the Casimir force $F^{\operatorname{expt}}(a)$ between a sphere and a plate, as a function of separation distance $a$ between them.

In each experiment, there are several sources of the absolute systematic errors $\Delta_{i}^{s} \Pi^{\operatorname{expt}}(a)$ and corresponding relative systematic errors $\delta_{i}^{s} \Pi^{\operatorname{expt}(a)}$ $=\Delta_{i}^{s} \Pi^{\operatorname{expt}}(a) /\left|\Pi^{\operatorname{expt}}(a)\right|$, where $1 \leqslant i \leqslant J$ (see Secs. IV and $\mathrm{V}$ for the description of specific experiments). It is necessary to stress that both in metrology and in all natural sciences (physics, chemistry, biology, etc.) the term systematic error is used in two different meanings (Rabinovich, 2000). According to the first meaning, a systematic error is some bias in the measurement which always makes the measured value higher or lower than the true value. Such systematic errors in the measurement results are usually removed using some known process, i.e., through a calibration. They can also be taken into account as corrections (see the description of the calibration procedure and an example of correction in the measurement of the Casimir force discussed in Sec. IV.A). The systematic errors in this understanding are often called systematic deviations. Below it is assumed that the experimental data under consideration are already free of such deviations.

Another meaning, which is used below, defines the systematic errors as the errors of a calibrated measurement device. The errors of some theoretical formula used to convert the directly measured quantity into the indirectly measured one (see Sec. IV.B) are also considered as systematic. In accordance with common understanding, the error of a calibrated device is the smallest fractional division on the scale of this device. In the limits of this range the systematic errors are considered as random quantities characterized by a uniform distribution. Because of this, the total relative systematic error is (Rabinovich, 2000)

$$
\begin{aligned}
& \delta^{S} \Pi^{\operatorname{expt}}(a) \\
& \quad=\min \left\{\sum_{i=1}^{J} \delta_{i}^{s} \Pi^{\operatorname{expt}}(a), k_{\beta}^{(J)} \sqrt{\sum_{i=1}^{J}\left[\delta_{i}^{s} \Pi^{\operatorname{expt}}(a)\right]^{2}}\right\},
\end{aligned}
$$

where $\beta$ is the chosen confidence level and $k_{\beta}^{(J)}$ is the tabulated coefficient depending on $\beta$ and on the number of sources of systematic errors $J$ in the experiment under consideration (Rabinovich, 2000). In precise experiments, errors should be determined at a confidence level 
$\beta \geqslant 95 \%$. However, in many Casimir force measurements only a $67 \%$ confidence level is used (see Sec. IV). Equation (77) can be written equivalently in terms of the absolute errors.

In precise experiments (see Secs. IV and V) it is common to perform several sets of measurements, say $n$, within the same separation region $\left(a_{\min }, a_{\max }\right)$. This is done in order to decrease the random error and to narrow the confidence interval. Each set consists of pairs $\left[a_{i}, \Pi^{\operatorname{expt}}\left(a_{i}\right)\right]$, where $1 \leqslant i \leqslant i_{\max }\left(i_{\max }\right.$ is different for different experiments). All measurement data can be represented as pairs $\left[a_{i j}, \Pi^{\operatorname{expt}}\left(a_{i j}\right)\right]$, where $1 \leqslant j \leqslant n$. If separations with fixed $i$ are approximately the same in all sets of measurement (i.e., $a_{i j} \approx a_{i}$ ), the mean and the variance of the mean at each point $a_{i}$ are obtained in the standard way (Rabinovich, 2000),

$$
\begin{aligned}
& \bar{\Pi}_{i}^{\text {expt }}=\frac{1}{n} \sum_{j=1}^{n} \Pi^{\operatorname{expt}}\left(a_{i j}\right), \\
& s_{\bar{\Pi}_{i}}^{2}=\frac{1}{n(n-1)} \sum_{j=1}^{n}\left[\Pi^{\operatorname{expt}}\left(a_{i j}\right)-\bar{\Pi}_{i}^{\text {expt }}\right]^{2} .
\end{aligned}
$$

If separations with fixed $i$ are different in different sets of measurements (i.e., with different $j$ ), the calculation of variance demands a more sophisticated procedure (Decca, López, Fischbach, et al., 2005; Klimchitskaya, Chen, et al., 2006).

Direct calculations show that the mean values $\bar{\Pi}_{i}^{\text {expt }}$ are uniform, i.e., change smoothly with the change of $i$. The variances of the mean $s_{\bar{\Pi}_{i}}$ are, however, not uniform. To smooth them, a special procedure is used in statistics (Cochran, 1954; Brownlee, 1965). The application of this procedure allows one to replace $s_{\bar{\Pi}_{i}}$ with the smooth function of separation $s_{\bar{\Pi}}^{-}(a)$ (Klimchitskaya, Chen, et al., 2006). Then the random absolute error at a separation distance $a$ can be calculated at a chosen confidence level $\beta$,

$$
\Delta^{r} \Pi^{\operatorname{expt}}(a)=s_{\Pi}^{-}(a) t_{(1+\beta) / 2}(n-1) .
$$

Here the value of $t_{p}(f)$ can be found in the tables for Student's $t$ distribution [see, e.g., Brandt (1976)].

To find the total experimental error of $\Pi^{\operatorname{expt}}(a)$, one should combine the random and systematic errors. In so doing it is assumed that the random error is characterized by the Student distribution which approaches to the normal distribution with the increasing number of the measurement sets. The systematic error is assumed to be described by a uniform distribution. There are different methods in statistics to obtain this combination (Rabinovich, 2000). A widely used method is based on the value of the quantity $r(a)=\Delta^{s} \Pi^{\operatorname{expt}}(a) / s_{\Pi}^{-}(a)$. According to this method, at all $a$, where $r(a)<0.8$, the contribution from the systematic error is negligible and $\Delta^{t} \Pi^{\operatorname{expt}}(a)=\Delta^{r} \Pi^{\operatorname{expt}}(a)$ at a $95 \%$ confidence level [in so doing $\Delta^{r} \Pi^{\operatorname{expt}}(a)$ is also calculated at $95 \%$ confidence using Eq. (79)]. If the inequality $r(a)>8$ is valid, the random error is negligible and $\Delta^{t} \Pi^{\operatorname{expt}}(a)=\Delta^{s} \Pi^{\operatorname{expt}}(a)$ with
$\Delta^{s} \Pi^{\operatorname{expt}}(a)$ calculated at $95 \%$ confidence. In the separation region, where $0.8 \leqslant r(a) \leqslant 8$, a combination of errors is performed using the rule

$$
\Delta^{t} \Pi^{\operatorname{expt}}(a)=q_{\beta}(r)\left[\Delta^{r} \Pi^{\operatorname{expt}}(a)+\Delta^{s} \Pi^{\operatorname{expt}}(a)\right],
$$

where the coefficient $q_{\beta}(a)$ at a $\beta=0.95$ confidence level varies between 0.71 and 0.81 depending on $r(a)$. Being conservative, one can use $q_{0.95}(r)=0.8$.

It is important that the total experimental error $\Delta^{t} \Pi^{\operatorname{expt}}(a)$ and the corresponding total relative error $\delta^{t} \Pi^{\operatorname{expt}}(a)=\Delta^{t} \Pi^{\operatorname{expt}}(a) /\left|\Pi^{\operatorname{expt}}(a)\right|$ characterize the precision of an experiment in its own without comparison with any theory. Note also that in metrology an experiment is called precise if the random error is much smaller than the systematic error (Rabinovich, 2000). In Casimir force measurements performed up to date, only one experiment (Decca et al., 2007a, 2007b) satisfies this rigorous criterion.

\section{Comparison of experiment with theory}

The theoretical values $\Pi^{\text {theor }}(a)$ (the pressure between the plates or the force between the sphere and the plate) computed using the Lifshitz theory also contain some errors. If the Casimir force between a sphere and a plate is calculated, one of the theoretical errors is caused using the proximity force approximation. To be conservative, one can set the respective relative error to $a / R$, i.e., to its maximum possible value (see Sec. II.B). Another error is due to the uncertainty in the optical data used. It can be conservatively estimated to be equal to $0.5 \%$ over the entire measurement range (Chen, Klimchitskaya, Mohideen, and Mostepanenko, 2004). The total theoretical error can be computed using Eq. (77) adapted for theoretical errors. Note that when using different sets of optical data one can arrive at different computational results for $\Pi^{\text {theor }}(a)$. This has resulted in statements in the literature [see, e.g., Pirozhenko et al. (2006) and Capasso et al. (2007)] that if different sets of optical data obtained for different samples lead to deviations in the Casimir force equal to, say, around $5 \%$, then the comparison of theory with experiment on a better level is impossible. These statements are, however, incorrect from the point of view of measurement theory (Decca et al., 2007b). The point to note is that here we are dealing with methods for the verification of hypotheses. The procedures for such verifications are well developed in statistics. Different hypotheses correspond to using different theoretical approaches (for instance, based on the Drude or plasma model description of conduction electrons in metals or the inclusion or the neglect of the small dc conductivity of dielectrics at nonzero temperature) with different sets of optical data available. The comparison of different hypotheses with experiment can be performed by plotting the respective theoretical bands as functions of separation (the width of the band is determined by the total theoretical error) and the experimental data with their absolute errors on one graph [see, e.g., Fig. 11(a) in Sec. IV.B]. If the theoretical band does not overlap with the experimental 
data, including their errors, over a wide separation range, it is experimentally excluded and the corresponding hypothesis must be rejected. It is important to note that the data and theory are not compared at just one point but over a wide separation region where the distance dependence is nonlinear. The set of optical data which contradicts the experimental results, when combined with any theoretical approach, should be considered as irrelevant to the actual properties of the film used in the measurement.

Another method to compare theory with experiment is the consideration of the confidence interval for the random quantity $\Pi^{\text {theor }}\left(a_{i}\right)-\bar{\Pi}^{\operatorname{expt}}\left(a_{i}\right)$ (Decca, López, Fischbach, et al., 2005; Chen, Mohideen, et al., 2006). In doing so, one should take into account that this comparison is done at the separation distances $a_{i}$ measured with some error $\Delta a$. Thus, the theoretical value $\Pi^{\text {theor }}\left(a_{i}\right)$ at this point can be known only with the relative error $\alpha \Delta a / a_{i}$ (Iannuzzi, Gelfand, et al., 2004). Here $\alpha=3$ for the force between a sphere and a plate and $\alpha=4$ for the pressure between the two plates. This error, although not connected with the theoretical approach used, should be included as part of the theoretical errors in Eq. (77). Note that at short separations it results in a major contribution to the total theoretical error $\Delta^{t} \Pi^{\text {theor }}$. Then the absolute error of the difference between theory and experiment $\Xi_{\Pi}(a)$ at a $95 \%$ confidence can be calculated using Eq. (77) with $J=2$ and $k_{0.95}^{(2)}=1.1$,

$$
\begin{aligned}
\Xi_{\Pi}(a)= & \min \left\{\Delta^{t} \Pi^{\operatorname{expt}}(a)+\Delta^{t} \Pi^{\text {theor }}(a),\right. \\
& \left.1.1 \times \sqrt{\left[\Delta^{t} \Pi^{\operatorname{expt}}(a)\right]^{2}+\left[\Delta^{t} \Pi^{\text {theor }}(a)\right]^{2}}\right\} .
\end{aligned}
$$

The confidence interval for the quantity $\Pi^{\text {theor }}(a)$ $-\bar{\Pi}^{\operatorname{expt}}(a)$ at a $95 \%$ confidence level is given by $\left[-\Xi_{\Pi}(a), \Xi_{\Pi}(a)\right]$. When one compares a theoretical approach with experimental data, the differences between the theoretical and mean experimental values of $\Pi$ may or may not belong to this interval. A theoretical approach for which not less than $95 \%$ of the differences $\Pi^{\text {theor }}(a)-\bar{\Pi}^{\operatorname{expt}}(a)$ belong to the interval $\left[-\Xi_{\Pi}(a), \Xi_{\Pi}(a)\right]$ within any separation subinterval $\left[a_{1}, a_{2}\right]$ of the entire measurement range is consistent with the experiment. In this case the measure of agreement between experiment and theory is given by $\Xi_{\Pi}(a) /\left|\bar{\Pi}^{\operatorname{expt}}(a)\right|$. On the contrary, if for some theoretical approach a subinterval $\left[a_{1}, a_{2}\right]$ exists, where almost all differences $\Pi^{\text {theor }}(a)-\bar{\Pi}^{\operatorname{expt}}(a)$ are outside of the confidence interval $\left[-\Xi_{\Pi}(a), \Xi_{\Pi}(a)\right]$, this theoretical approach is excluded by experiment at separations from $a_{1}$ to $a_{2}$ at a $95 \%$ confidence level. If the theoretical approach (hypothesis) is excluded by experiment at a $95 \%$ confidence level, the probability that it is true is at most $5 \%$. It may happen that several theoretical approaches $i=1,2, \ldots$ are consistent with experiment, i.e., not less than $95 \%$ of the differences $\Pi_{i}^{\text {theor }}(a)-\bar{\Pi}^{\operatorname{expt}}(a)(i=1,2, \ldots)$ belong to the confidence interval $\left[-\Xi_{\Pi}(a), \Xi_{\Pi}(a)\right]$ (such situations are considered in Secs. IV and V). The statistical criteria used do not allow an indication of the probability of the event that one of these approaches or all of them are false. The rejection of some of the experimentally consistent approaches can be done on a theoretical basis only. For example, if the measurement is performed at room temperature and both theoretical computations done at $T$ $=0$ and $300 \mathrm{~K}$ are consistent with the data, the computation at $T=300 \mathrm{~K}$ can be considered as preferable. In fact, to reliably discriminate between the two experimentally consistent theoretical approaches, more exact measurements are desirable. From the above it becomes clear that the described statistical criteria are somewhat asymmetric. They provide solid grounds for rejection of the experimentally inconsistent approaches at a high confidence level but do not permit the claim of the experimental confirmation of some consistent approach at the same high confidence. These general criteria are illustrated in Secs. IV and V using the examples of different Casimir force measurements (see Figs. 12, 13, and 21). It would be interesting to apply more sophisticated statistical methods, for instance, Bayesian (Berger, 1993; Carlin and Louis, 2000; Lehmann and Romano, 2005) for the data analysis of force-distance relations resulting from the Casimir force measurements. Until now, however, such methods have not been used devoted to the experimental study of the Casimir effect.

It is notable that conclusions concerning consistency or rejection of a hypothesis do not depend on the method of comparison used (see discussion of different situations in Secs. IV-VI). The half width of the confidence interval $\Xi_{\Pi}(a)$ has very little dependence on the theoretical approach used (i.e., on the hypothesis to be verified). It usually results from the experimental errors in the measurement of the separation distance and the Casimir force or the pressure.

\section{CASIMIR EXPERIMENTS WITH METALLIC TEST BODIES}

\section{A. Measurements of the Casimir force using an atomic force microscope}

Recent interest in the experimental investigation of the Casimir effect has its beginning in the experiment by Lamoreaux (1997). This experiment, however, contains several uncertainties that do not permit one to consider it as precise and definitive (see the discussion in Sec. IV.C.1). The first precise and definitive direct measurements of the Casimir force between a metal-coated sphere and a plate were performed by Mohideen and Roy (1998), Roy et al. (1999), and Harris et al. (2000) in three successive experiments using an AFM operated in vacuum. In these experiments the fundamental requirements for the measurement of the Casimir force proposed by Sparnaay $(1958,1989)$, such as (i) clean surfaces for the test bodies, (ii) precise and reproducible measurement of the separation distance between them, and (iii) low electrostatic potential differences, were met for the first time [historical survey of the Casimir force measurements performed before 1990 is given by Bordag et al. (2001)]. They introduced the now standard use 


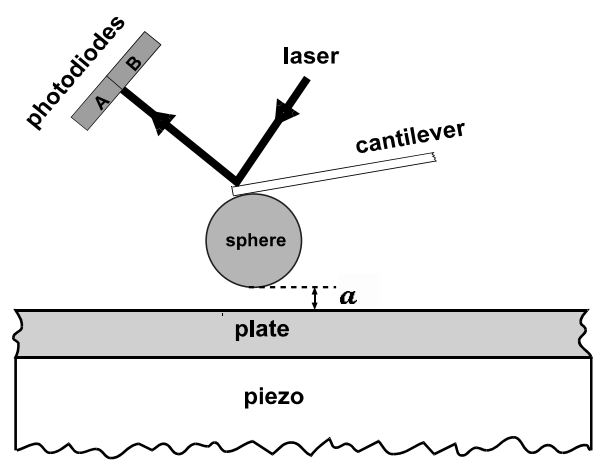

FIG. 4. Schematic diagram of the measurement of the Casimir force using an atomic force microscope.

of metal-coated polystyrene spheres, which have the unique advantages of being extremely light weight, perfectly smooth (made from liquid phase), and having very low eccentricity (less than parts per thousand) as one of the surfaces in Casimir force measurements. These measurements also pioneered the independent determination of the roughness-induced average separation distances at contact, the cantilever calibration, and the potential differences between the two surfaces using the electrostatic force. This allowed a careful comparison to the theory and a precise study of the effect of the metal conductivity (also in terms of the skin depth) and the surface roughness which previously could not be done.

A schematic diagram of the experimental setup for the measurement of the Casimir force using the AFM is shown in Fig. 4. The Casimir force between a sphere of radius $R$ and a plate causes the cantilever to flex. This flexing is detected by the deflection of a laser beam leading to a difference signal $S_{\text {def }}$ between photodiodes $A$ and $B$ which was calibrated by means of the electrostatic force between the sphere and the plate. For this purpose various voltages $V$ were applied to the plate while the sphere remained grounded. The measurements of the electrostatic forces were performed at separations $a$ larger than a few micrometers where the contribution from the Casimir force is negligibly small. The exact expression for the electrostatic force in sphere-plate configuration is given by (Smythe, 1950)

$$
\begin{aligned}
F_{\mathrm{el}}(a) & =2 \pi \epsilon_{0}\left(V-V_{0}\right)^{2} \sum_{n=1}^{\infty} \frac{\operatorname{coth} \alpha-n \operatorname{coth} n \alpha}{\sinh n \alpha} \\
& \equiv X(a)\left(V-V_{0}\right)^{2},
\end{aligned}
$$

where $\cosh \alpha=1+a / R, V_{0}$ is the residual voltage on the sphere and $\epsilon_{0}$ is the permittivity of the vacuum. From a comparison of theoretical expression (82) with the experimental data, the separation on contact $a_{0}$ and the residual potential difference between the test bodies were determined. Finally the absolute separations were found from

$$
a=a_{0}+a_{\text {piezo }}+S_{\text {def }} m,
$$

where the movement $a_{\text {piezo }}$ of the piezoelectric tube on which the plate is mounted was calibrated by an optical

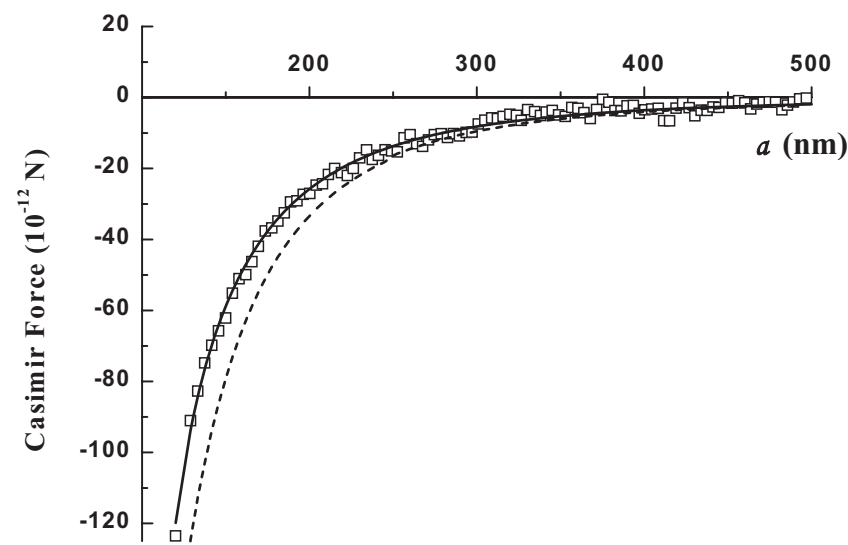

FIG. 5. Comparison of experiment and theory. Measured mean Casimir force between $\mathrm{Al}$ surfaces versus separation is shown as open squares. The theoretical Casimir force with corrections due to skin-depth and surface roughnesses and for ideal metal surfaces is shown by the solid and dashed lines, respectively.

fiber interferometer (Chen and Mohideen, 2001). As the piezo was moved with continuous periodic triangular voltages, the nonlinearities can be precisely accounted for through interferometric calibration. The last term in Eq. (83) is due to the decrease in the separation distance from the flexing of the cantilever in response to any force. Note that $S_{\text {def }}<0$ for an attractive force. The deflection coefficient $m$ was found from electrostatic calibration.

To measure the Casimir force between the sphere and the plate, they are both grounded together with the AFM. The plate is then moved toward the sphere using the piezo and the corresponding photodiode difference signal is measured. It is converted into the respective deflection of the cantilever tip and by use of Hooke's law into the values of the Casimir force $S_{\text {def }} k m$, where $k$ is the spring constant (see below for the results of electrostatic calibration and the values of the main parameters). In the first two measurements using the AFM by Mohideen and Roy (1998) and Roy et al. (1999), the sphere and the plate were coated with about $300 \mathrm{~nm}$ of Al. To prevent rapid oxidation of $\mathrm{Al}$ it was sputter coated with thin $\mathrm{Au} / \mathrm{Pd}$ layers. In the first experiment (Mohideen and Roy, 1998; Klimchitskaya et al., 1999) it was demonstrated that the Casimir force between real metal surfaces deviates significantly from the Casimir prediction made for ideal metal surfaces. In Fig. 5 the measured data for the Casimir force, as a function of separation distance $a$, are shown as open squares. The dashed line indicates the Casimir force (19) between an ideal metal plate and an ideal metal sphere of $R=100 \pm 2 \mu \mathrm{m}$ radius used in the experiment. The solid line shows the theoretical Casimir force calculated with account of the corrections due to the skin depth and the surface roughness. This force was found using the fourth-order perturbation theory in the relative skin depth $\delta_{0} / a$ defined in Sec. II.D.1. The perturbation theory (Klimchitskaya et al., 1999; Bezerra, Klimchitskaya, and 
Mostepanenko, 2000), when applied in the appropriate region of the plasma model, leads to approximately the same results as the Lifshitz formula at zero temperature (first measurements using the AFM were not of sufficient precision to measure the thermal effect at $T$ $=300 \mathrm{~K}$ ). The Casimir force including the effect of both the skin depth and roughness corrections was calculated using Eq. (75) and the PFA (16). As shown in Fig. 5, the solid line is in good agreement with data, thus, demonstrating the role of skin depth and roughness corrections.

We now consider the most precise, third, measurement of the Casimir force between metal surfaces by means of an AFM (Harris et al., 2000). The use of a thin $\mathrm{Au} / \mathrm{Pd}$ coating on the top of the $\mathrm{Al}$ in the first two experiments prevented a complete theoretical treatment of the properties of the real metal surfaces. Because of this, in the third experiment, Au layers of $86.6 \pm 2 \mathrm{~nm}$ thickness were coated on both the plate and the spherical surfaces. Such a coating is sufficient to reproduce the properties of an infinitely thick metal (see Sec. III.B.2). The sphere diameter (including the metal coating) was measured using the scanning electron microscope to be $191.3 \pm 0.5 \mu \mathrm{m}$ (independent calibration of the scanning electron microscope was done with interferometrically calibrated AFM piezo standards). The cantilever was calibrated and the residual potential between the surfaces was measured using the electrostatic force at separations larger than $3 \mu \mathrm{m}$ with voltages $V$ from -3 to $3 \mathrm{~V}$ applied to the plate. As a result the following values were obtained: $m=8.9 \pm 0.3 \mathrm{~nm}$ per unit deflection signal, $\mathrm{km}=0.386 \pm 0.003 \mathrm{nN}$ per unit deflection signal, $a_{0}$ $=32.7 \pm 0.8 \mathrm{~nm}$, and the residual potential on the grounded sphere $V_{0}=3 \pm 3 \mathrm{mV}$. At separations of about $60 \mathrm{~nm}$ this residual potential leads to forces which are only $0.075 \%$ of the Casimir force. At $a=100 \mathrm{~nm}$ the contribution of the residual electric force increases up to $0.17 \%$ and at $a=160 \mathrm{~nm}$ up to $0.36 \%$ of the Casimir force.

The magnitudes of the residual potential and separation on contact were shown to be independent of the distances where the measurements of the electric force were performed. The electric force corresponding to the residual potential difference has been subtracted from the measured total force to obtain the pure Casimir force. This is in fact a correction made to remove the systematic deviation due to the residual potential difference, as discussed in Sec. III.C.1. From the above values it becomes clear that the electrostatic residuals are negligible in comparison with the Casimir force. The electric force due to the patch potentials contributes only $0.23 \%$ and $0.008 \%$ of the Casimir force at separations $a=62$ and $100 \mathrm{~nm}$, respectively (Chen, Klimchitskaya, Mohideen, and Mostepanenko, 2004). Recently it was claimed that the residual potential $V_{0}$ from the electrostatic calibration in sphere-plate configuration is separation dependent (Kim et al., 2008). They used a Aucoated sphere of $30.9 \mathrm{~mm}$ radius at separations of a few tens of nanometers above a Au-coated plate. On the

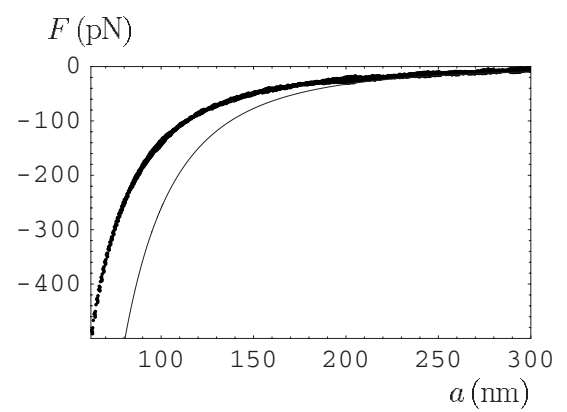

FIG. 6. Comparison of experiment with ideal metal theory. The measured mean Casimir force between Au surfaces versus separation is shown as dots. Theoretical Casimir force for ideal metal surfaces is shown by the solid line.

basis of these measurements a reanalysis of the independence of $V_{0}$ on separation in the measurements of the Casimir force by means of an atomic force microscope and a micromachined oscillator (see Sec. IV.B) was invited. Decca et al. (2009) demonstrated, however, that for a centimeter-size spherical lens at such short separations from the plate, the electrostatic force law used by Kim et al. (2008) is not applicable due to the inevitable deviations from a perfect spherical shape of the mechanically polished and ground surface and the presence of dust or other impurities. Because of this, the observed anomalies in the electrostatic calibration are not directly relevant to the experimental results considered here.

The averaged Casimir force measured from 27 scans is reproduced in Fig. 6 as dots within the measurement range from 62 to $300 \mathrm{~nm}$. In the same figure the solid line represents the Casimir force between ideal metal interacting surfaces. This once again demonstrates the difference between ideal metal surfaces and real materials.

The experimental errors in the experiment by Harris et al. (2000) were reanalyzed by Chen, Klimchitskaya, Mohideen, and Mostepanenko (2004). The maximum value of the systematic error at a $95 \%$ confidence level was shown to be $\Delta^{s} F^{\text {expt }}=2.7 \mathrm{pN}$ and of the random error $\Delta^{r} F^{\text {expt }}=5.8 \mathrm{pN}$ over the entire measurement range. Using the combination rule in Eq. (80), the total experimental error of the force measurements is given by $\Delta^{t} F^{\text {expt }}=6.8 \mathrm{pN}$. It does not depend on separation. The relative error varies from $1.5 \%$ to $2 \%$ when the separation increases from 63 to $72 \mathrm{~nm}$. It increases to $4.8 \%$ and $30 \%$ when separation increases to 100 and $200 \mathrm{~nm}$, respectively. The error in the measurements of absolute separations was found to be $\Delta a=1 \mathrm{~nm}$. All errors are determined at a $95 \%$ confidence level. The experimental data over the separation region from 63 to $100 \mathrm{~nm}$ are shown in Fig. 7 as crosses, where the sizes of the errors in the separation and force measurements are given in true scales. To make the figure readable, only every second data point is presented. We underline that the measurement of the Casimir force described above is independent in the sense that no fit to any theory of dispersion forces has been used. The above procedures used a fit only to the well-understood electric force in 


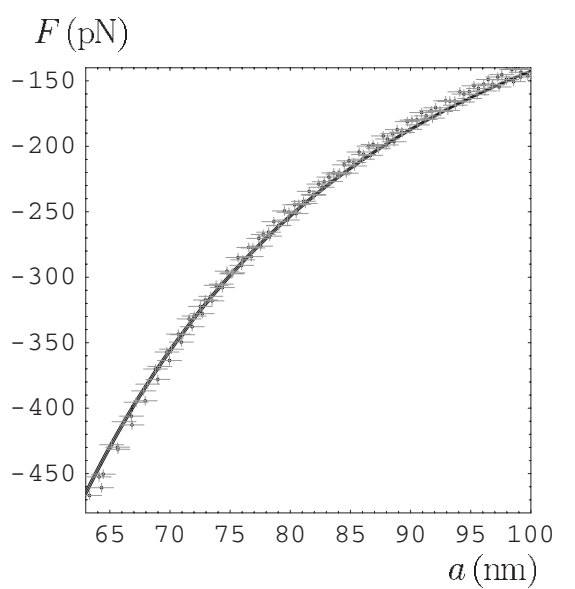

FIG. 7. Comparison of experiment and theory. The measured mean Casimir force between Au surfaces versus separation is shown as crosses. The arms of the crosses represent in true scale absolute errors determined at $95 \%$ confidence. Theoretical Casimir force calculated using the generalized plasmalike model is shown by the solid band.

order to find the values of some parameters such as the separation on contact $a_{0}$, the residual potential difference $V_{0}$, and deflection coefficient $m$.

A theoretical calculation of the Casimir force by Harris et al. (2000) was performed using the Lifshitz formula at zero temperature [Eq. (12)] and the PFA [Eq. (16)]. The dielectric permittivity of $\mathrm{Au}$ along the imaginary frequency axis was obtained using the first approach described in Sec. III.A.1, i.e., with the optical data extrapolated to low frequencies by means of the Drude model (the dashed line in Fig. 1). The character of roughness was investigated from the analysis of the AFM images of the surfaces of Au films. A typical AFM image of surface topography is presented in Fig. 8. As shown in this figure, the roughness is mostly represented by the stochastically distributed distortions with typical heights of about $2-4 \mathrm{~nm}$ and rare pointlike peaks with typical heights up to $16 \mathrm{~nm}$. Zero-roughness levels on both the

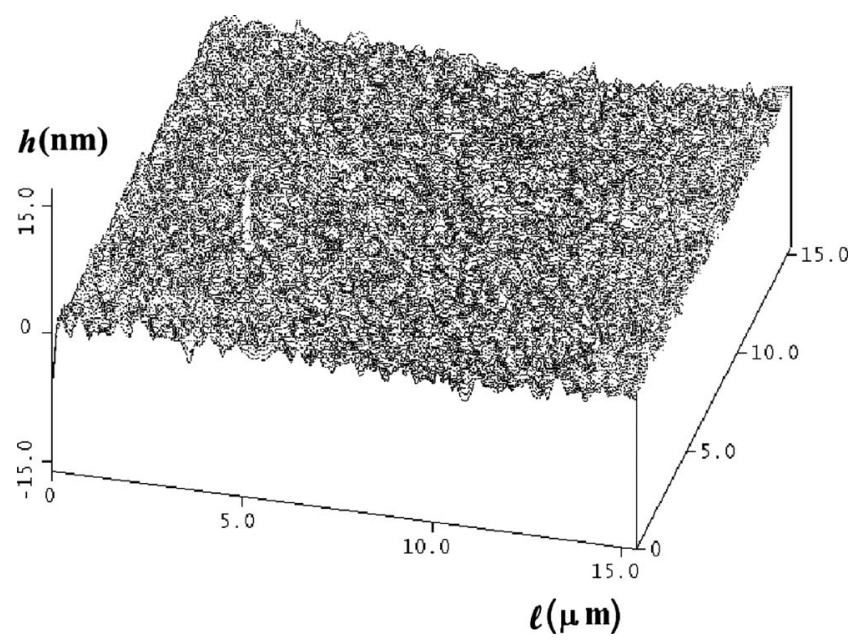

FIG. 8. Typical atomic force microscope image of the Au coating on the plate with roughness height $h$ and lateral scale $l$. plate and the sphere were found from Eq. (65) to be $H_{0}=2.734 \mathrm{~nm}$ (Chen, Klimchitskaya, Mohideen, and Mostepanenko, 2004). The rms variance defined in Eq. (71) is $\delta_{\mathrm{st}}=1.18 \mathrm{~nm}$. The role of roughness in this experiment is very small. Even at the shortest separation $a$ $=62 \mathrm{~nm}$ roughness contributes only $0.24 \%$ of the Casimir force. The diffraction-type and correlation effects discussed in Sec. III.B.1 were shown to contribute less than one-tenth of this value (Chen, Klimchitskaya, Mohideen, and Mostepanenko, 2004).

Comparison of the data with the Lifshitz theory at $T$ $=0$ taking the surface roughness into account demonstrated good agreement over the entire measurement range (Harris et al., 2000; Chen, Klimchitskaya, Mohideen, and Mostepanenko, 2004). However, as discussed in Sec. II.D.1, the use of the Drude model at low frequencies presents serious difficulties. In addition, according to Sec. III.A.2, the use of the zero-temperature Lifshitz formula for the interpretation of the experiment performed at $T=300 \mathrm{~K}$ is open to discussion. In Sec. III.A.1 a second approach to the calculation of $\mathrm{Au}$ dielectric permittivity along the imaginary frequency axis, was described. It is based on the plasmalike dielectric permittivity with the inclusion of the relaxation due to the core electrons alone (the solid line in Fig. 1). This approach can be used in combination with the Lifshitz formula at $T \neq 0$ with no thermodynamic inconsistencies. Calculations of the Casimir force in the experimental configuration of Harris et al. (2000) at $T=300 \mathrm{~K}$ using the generalized plasma model (i.e., with the dielectric permittivity given by the solid line in Fig. 1) were performed by Klimchitskaya et al. (2007a). The Casimir force obtained, as a function of separation, is shown by the solid band in Fig. 7. The width of this band indicates the total theoretical error calculated at a 95\% confidence level as described in Sec. III.C.2, taking into account the error of the PFA and uncertainties in the optical data of Au related to the interband transitions. As shown in Fig. 7, the theoretical approach using the generalized plasmalike permittivity is also in good agreement with the data. Within the separation region from 63 to $72 \mathrm{~nm}$, where the relative total experimental error determined at a $95 \%$ confidence level varies from $1.5 \%$ to $2 \%$, all experimental crosses overlap with the theoretical band. From this one can conclude that the measure of agreement between experiment and theory at separation distances from 63 to $72 \mathrm{~nm}$ also varies from $1.5 \%$ to $2 \%$ of the measured force.

Thus, both theoretical approaches using the complete optical data for Au extrapolated to low frequencies by the Drude model and the Lifshitz formula at $T=0$ or the generalized plasmalike dielectric permittivity and the Lifshitz formula at $T=300 \mathrm{~K}$ are consistent with the experimental data by Harris et al. (2000). This is because at separations below $100 \mathrm{~nm}$, where the precision of this experiment is relatively high, the theoretical approaches used lead to almost coincident results. At such short separations approximately the same theoretical results are also obtained using the complete optical data ex- 
trapolated to zero frequency by the Drude model and the Lifshitz formula at $T=300 \mathrm{~K}$. However, at larger separation distances the calculational results obtained using the above two approaches at nonzero temperature become significantly different. Because of this, highprecision experiments at separations of a few hundred nanometers, considered next, are important for the understanding of the underlying physics.

\section{B. Precise determination of the Casimir pressure using a micromachined oscillator}

Microelectromechanical systems are well adapted for the investigation of small forces acting between closely spaced surfaces. One such system, a micromachined oscillator, was first used by Chan et al. (2001a, 2001b) to demonstrate the influence of the Casimir force on the static and dynamic properties of micromechanical systems (see Sec. IV.C.2).

Precise determination of the Casimir pressure between two parallel metallic plates by means of a micromachined oscillator was performed in three successive experiments by Decca et al. These experiments do not use the configuration of two parallel plates. The first experiment was made with a Au-coated sphere and $\mathrm{Cu}-$ coated plate (Decca, Fischbach, et al., 2003; Decca, López, et al., 2003, Decca et al., 2004). The second experiment with several improvements used both a Au-coated sphere and a plate (Decca, López, Fischbach, et al., 2005; Klimchitskaya et al., 2005). Further improvements implemented in the third experiment with a Au-coated sphere and Au-coated plate (Decca et al., 2007a, 2007b) made it the most precise and reliable measurement with metallic test bodies ever performed in the Casimir force measurements to date. Here we discuss the measurement scheme and the main physical results following from the third experiment by Decca et al. (2007a, 2007b).

In metrological terms this is an indirect measurement (Rabinovich, 2000) of the Casimir pressure between two Au-coated parallel plates. Note that the results of direct measurements are found just from the experiment. The results of indirect measurements are obtained with the help of calculations using the known equations which relate the quantity under consideration with some quantities measured directly. The Casimir force per unit area (pressure) was determined in these experiments dynamically by means of a micromechanical torsional oscillator consisting of a $500 \times 500 \mu \mathrm{m}^{2}$ heavily doped polysilicon plate suspended along one central planar axis by serpentine springs and a sphere of $R=151.3 \mu \mathrm{m}$ radius above it attached to an optical fiber (see Fig. 9). During the measurements, the separation distance between the sphere and the plate was varied harmonically, $\tilde{a}(t)=a$ $+A_{z} \cos \left(\omega_{r} t\right)$, where $\omega_{r}$ is the resonant angular frequency of the oscillator under the influence of the Casimir force $F_{s}(a, T)$ from the sphere and $A_{z} / a \ll 1$. The frequency $\omega_{r}$ is related to the natural angular frequency of the oscillator $\omega_{0}=2 \pi \times(713.25 \pm 0.02) \mathrm{Hz}$ by (Chan et al., 2001a, 2001b; Decca, Fischbach, et al., 2003; Decca, López, et al., 2003)

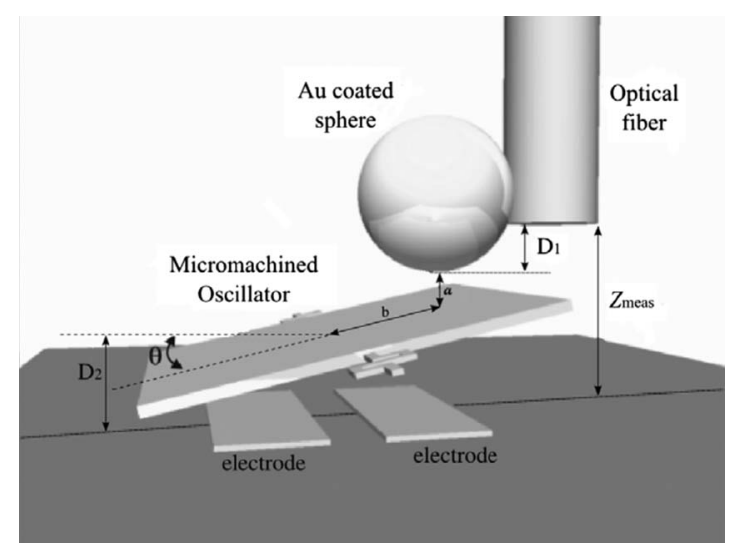

FIG. 9. Schematic diagram of the measurement of the Casimir force using a micromachined oscillator.

$$
\omega_{r}^{2}=\omega_{0}^{2}\left(1-\frac{b^{2}}{I \omega_{0}^{2}} \frac{\partial F_{s}(a, T)}{\partial a}\right),
$$

where $b$ is the lever arm, $I$ is the moment of inertia, and $b^{2} / I=1.2432 \pm 0.0005 \mu \mathrm{g}^{-1}$.

The frequency shift $\omega_{r}-\omega_{0}$ is directly measured and from Eq. (84) it leads to the calculated values of the Casimir force gradient $\partial F_{s} / \partial a$. Using the PFA (16) this gradient can be expressed through the effective Casimir pressure in the configuration of two parallel plates,

$$
P(a, T)=-\frac{\partial \mathcal{F}(a, T)}{\partial a}=-\frac{1}{2 \pi R} \frac{\partial F_{s}(a, T)}{\partial a} .
$$

Because of this, a direct measurement of the frequency shift caused by the Casimir force between a sphere and a plate results in an indirect measurement of the Casimir pressure in the configuration of two parallel plates.

The separation distance between the zero-roughness levels of Au layers on the plate and on the sphere (see Sec. III.B.1) is given by

$$
a=z_{\text {meas }}-\left(D_{1}+D_{2}\right)-b \theta .
$$

Here $z_{\text {meas }}$ is the separation between the end of the cleaved fiber and the platform and $\theta$ and $D_{1,2}$ are defined in Fig. 9. The lever arm $b$ was measured optically. The value of $\theta$ was determined by measuring the difference in capacitance between the plate and the right and left electrodes shown in Fig. 9. The value $D_{1}+D_{2}$ was measured as a part of the system calibration. This was done by the application of voltages $V$ to the sphere while the plate was grounded. The electric force between a sphere and a plate was measured in the static regime, with no harmonic variations of the separation distance between them at large separations $a>3 \mu \mathrm{m}$. The residual potential difference $V_{0}$ was found to be independent of separation [details of calibration procedures are presented by Decca, López, Fischbach, et al. (2005), and Decca et al. (2009)]. We emphasize that in the second (Decca, López, Fischbach, et al., 2005) and third (Decca et al., 2007a, 2007b) experiments of this series, contact between the sphere and the plate has not 


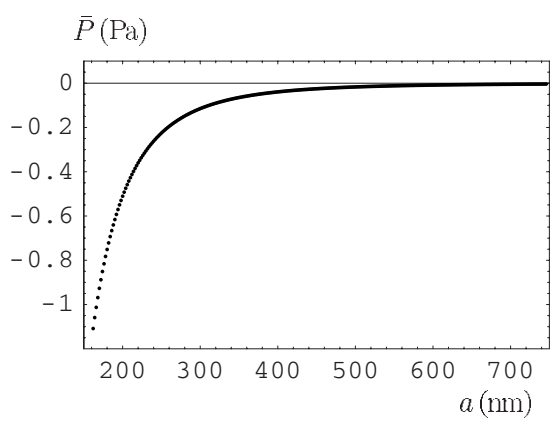

FIG. 10. The measured mean Casimir pressure between two parallel plates vs separation (dots).

been achieved and was not needed for the determination of absolute separations.

A set of 120 curves of $F_{\mathrm{el}}(a)$ was then used to fit the quantity $D_{1}+D_{2}$. Finally absolute separations $a$ were measured with an absolute error $\Delta a=0.6 \mathrm{~nm}$ determined at a $95 \%$ confidence. In contrast to previous measurements (Decca, Fischbach, et al., 2003; Decca, López, et al., 2003; Decca, et al. 2004; Decca, López, Fischbach, et al., 2005), in this experiment $n=33$ sets of measurements within a separation region from 162 to $746 \mathrm{~nm}$ were performed at almost the same intermediate separations $a_{i}$ $(1 \leqslant i \leqslant 293)$ in each set. This was made possible due to about $7 \%$ improvement in the vibrational noise, and an improvement in the interferometric technique used to yield the distance $z_{\text {meas }}$ (see Fig. 9). The use of a twocolor fiber interferometer yielded an error $\Delta z_{\text {meas }}$ $=0.2 \mathrm{~nm}$, and for every repetition of the Casimir pressure measurement it was possible to reposition the sample to within $\Delta z_{\text {meas }}$.

The mean values of the Casimir pressure (78) were determined from the frequency shifts using Eqs. (84) and (85) from data obtained in 33 sets of measurements. They are plotted in Fig. 10 as a function of separation. The random experimental error was found from Eqs. (78) and (79). It varies from $\Delta^{r} P^{\operatorname{expt}}=0.46 \mathrm{mPa}$ at $a$ $=162 \mathrm{~nm}$ to $\Delta^{r} P^{\text {expt }}=0.11 \mathrm{mPa}$ at $a=300 \mathrm{~nm}$. This value remains constant for separations up to $a=746 \mathrm{~nm}$. The systematic error in the indirect measurement of the pressure is determined by errors in the measurements of the resonant frequency and of the radius of the sphere, and also by the error introduced using the PFA. The latter is now related to experiment rather than to theory, because in this dynamic measurement PFA is a part of the experimental procedure of the determination of the Casimir pressure between the two parallel plates. According to the results presented in Sec. II.B, the error due to the use of PFA is less than $a / R$. Because of this, Decca et al. (2007a, 2007b) estimated this error conservatively as $a / R$. By combining all the above $J=3$ systematic errors at a $95 \%$ confidence using the statistical rule (77), the resulting systematic error was obtained. It is given by $\Delta^{s} P^{\operatorname{expt}}=2.12 \mathrm{mPa}$ at $a=162 \mathrm{~nm}$, decreases to $0.44 \mathrm{mPa}$ at $a=300 \mathrm{~nm}$, and then to $0.31 \mathrm{mPa}$ at $a$ $=746 \mathrm{~nm}$. Finally, the total experimental error $\Delta^{t} P^{\operatorname{expt}}(a)$ at a $95 \%$ confidence level was obtained using the statis- tical rule formulated in Sec. III.C.1. As a result, $\Delta^{t} P^{\operatorname{expt}}(a)=\Delta^{s} P^{\operatorname{expt}}(a)$ within the entire measurement range, i.e., the total experimental error is determined by only the systematic one. This means that the experiment by Decca et al. (2007a, 2007b) satisfies one of the main requirements imposed on precise experiments in metrology (Rabinovich, 2000). For now no other experiment in Casimir physics satisfies this requirement. The total relative experimental error varies from $0.19 \%$ at $a$ $=162 \mathrm{~nm}$ to $0.9 \%$ at $a=400 \mathrm{~nm}$ and to $9.0 \%$ at the largest separation $a=746 \mathrm{~nm}$.

To conclusively compare the experimental data with theory, the topography of metallic coatings both on the plate [body (1)] and on the sphere [body (2)] was investigated using an AFM. From the AFM images, the fraction of each surface area $v_{i}^{(1,2)}$ with the height $h_{i}^{(1,2)}$ was determined. It was found that for the plate $h_{i}^{(1)}$ varies from 0 to $18.35 \mathrm{~nm}$ and for the sphere $h_{i}^{(2)}$ varies from 0 to $10.94 \mathrm{~nm}$. Using Eq. (65) the zero-roughness levels on the plate and on the sphere are $H_{0}^{(1)}=9.66 \mathrm{~nm}$ and $H_{0}^{(2)}=5.01 \mathrm{~nm}$. The contribution of correlation and diffractionlike effects in the roughness correction was shown to be negligibly small (Decca, López, Fischbach, et al., 2005). The overall contribution of roughness was shown to be only $0.5 \%$ of the Casimir pressure at $a$ $=162 \mathrm{~nm}$ and it decreases with the increase of separation. To conclusively compare experimental data with theory, the resistivity of the $\mathrm{Au}$ layers as a function of temperature was also measured in the region from $T_{1}$ $=3$ to $400 \mathrm{~K}$. This has led to a slightly modified values of the Drude parameters, $\omega_{p}=8.9 \pm 0.1 \mathrm{eV}, \gamma=0.0357 \mathrm{eV}$, to be used in theoretical calculations (compare with $\omega_{p}$ $=9.0 \mathrm{eV}, \gamma=0.035 \mathrm{eV}$ in Sec. III.A.2).

The theoretical Casimir pressure as a function of separation between the plates was calculated using Eqs. (74) and (8) with different approaches to the determination of the dielectric permittivity along the imaginary frequency axis (see Sec. III.A.2) and using the Leontovich surface impedance (Sec. II.A). Comparison of experiment with theory was performed in two different ways described in Sec. III.C.2 (Decca et al., 2007a, 2007b). In Figs. 11(a) and 11(b) the mean experimental pressures are plotted as crosses within the separation region from 500 to $600 \mathrm{~nm}$. For other separation regions the situation is quite similar (Decca et al., 2007a). The arms of the crosses show the total experimental errors of separation and Casimir pressures in true scales determined at a $95 \%$ confidence level. The light-gray band in Fig. 11(a) shows the theoretical results computed using the generalized plasmalike dielectric permittivity, i.e., with $\varepsilon_{\mathrm{Au}}(i \xi)$, as given by the solid line in Fig. 1 . The width of the theoretical band in the vertical direction indicates the total theoretical error (Sec. III.C.2). It is equal to $0.5 \%$ of the calculated pressure and arises from the variations of the optical data of core electrons. Other factors, such as patch potentials, were shown to be negligible (Decca, López, Fischbach, et al., 2005). Note that when the experimental data with the errors, shown as crosses, are compared with the theoretical 

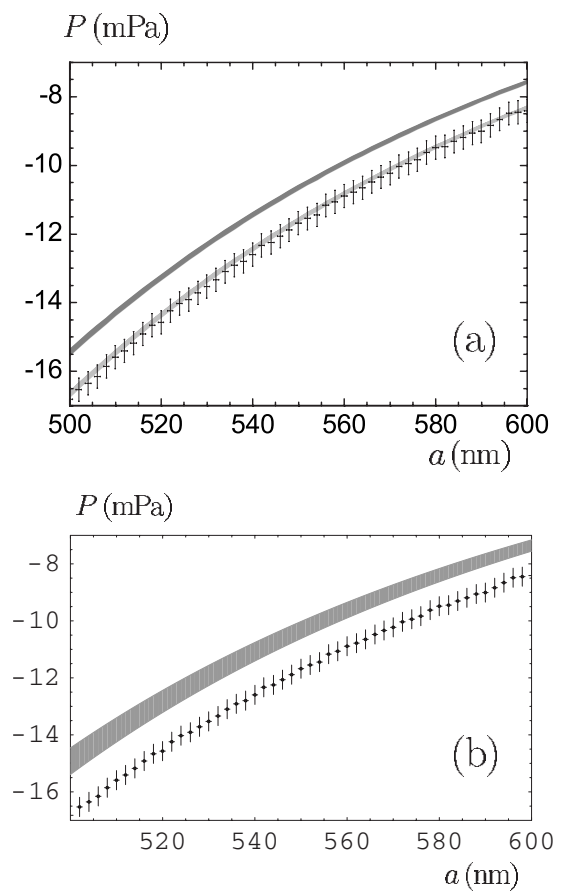

FIG. 11. Comparison of experiment and theory. The measured mean Casimir pressure together with the absolute errors in the separation and pressure vs separation is shown as crosses. (a) The theoretical Casimir pressure computed using the generalized plasmalike model and the optical data extrapolated by the Drude model is shown by the light-gray and dark-gray bands, respectively. (b) The theoretical Casimir pressure computed using different sets of optical data available vs separation is shown as the dark-gray band.

band computed for the entire measurement range, the errors $\Delta a$ in the measurement of separations are irrelevant to theory. The dark-gray band in Fig. 11(a) shows the theoretical results computed using the complete tabulated data extrapolated to low frequencies by means of the Drude model, i.e., with $\varepsilon_{\mathrm{Au}}(i \xi)$ shown as the dashed line in Fig. 1. As shown in Fig. 11(a), all data crosses overlap with the light-gray band but are separated by a gap from the dark-gray band. This means that the theory using the generalized plasmalike permittivity is consistent with experiment within the limits of the experimental error. At the same time, a theory using the complete optical data extrapolated by the Drude model is excluded by the data at a $95 \%$ confidence level.

As mentioned in Sec. III.C.2, the choice of different sets of Drude parameters may lead to an up to $5 \%$ variation in the theoretical values of the Casimir pressure (Pirozhenko et al., 2006). According to Sec. III.C.2, the hypothesis that the thin films used in the experiment possess such unusual Drude parameters is not supported by the data. Nevertheless, the disagreement between the Drude model approach and the experimental data only increases if some of the alternative Drude parameters, as considered by Pirozhenko et al. (2006), are used. As an illustration, in Fig. 11(b) the computation results obtained using the Drude model approach with $\omega_{p}$ varying from 6.85 (Pirozhenko et al., 2006) to $9.0 \mathrm{eV}$ are plotted
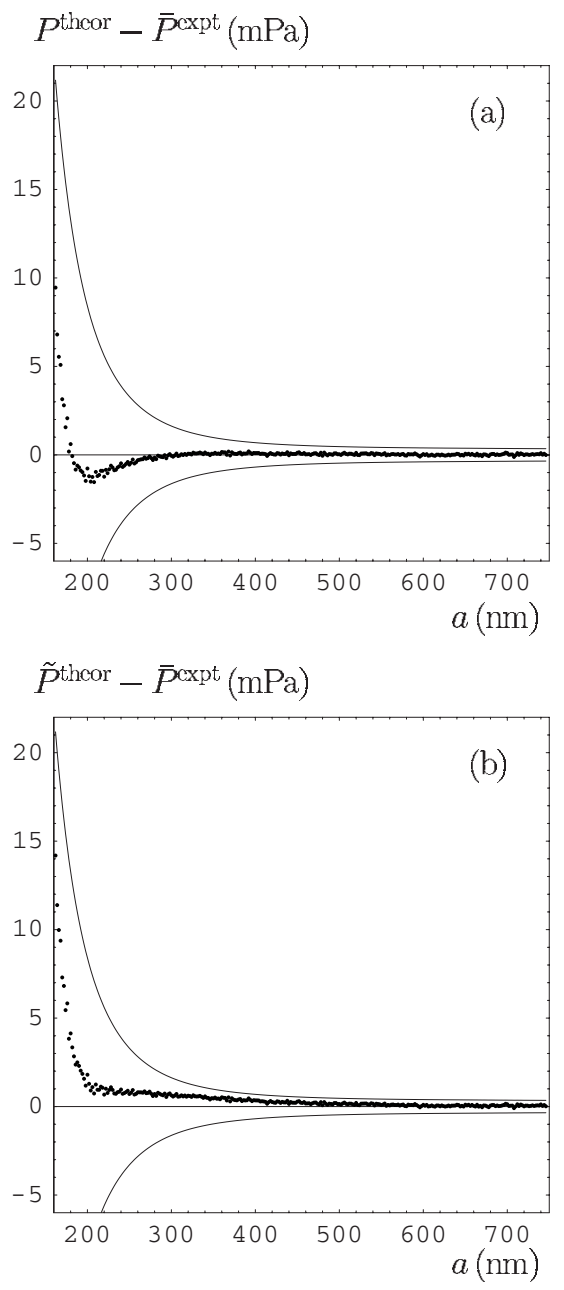

FIG. 12. Comparison of experiment and theory. The difference between theoretical and mean experimental Casimir pressures vs separation (dots) and the $95 \%$ confidence intervals (solid lines). The theoretical Casimir pressures are computed (a) using the generalized plasmalike model and (b) using the Leontovich surface impedance approach.

as the dark-gray band. Note that the values of the relaxation parameter only slightly influence the Casimir pressure. As shown in Fig. 11(b), the use of any alternative value of $\omega_{p}$ makes the disagreement between the Drude model approach and the data even more acute.

Another way to compare theory with experiment considered in Sec. III.C.2 is illustrated in Fig. 12, where the differences $P^{\text {theor }}\left(a_{i}\right)-P^{\operatorname{expt}}\left(a_{i}\right)$ are shown as dots and solid lines indicate the $95 \%$ confidence interval $\left[-\Xi_{P}(a), \Xi_{P}(a)\right]$ for the quantity $P^{\text {theor }}\left(a_{i}\right)-P^{\operatorname{expt}}\left(a_{i}\right)$ (Decca et al., 2007b). The values of $P^{\text {theor }}$ in Fig. 12(a) are computed using the generalized plasmalike model, whereas the values of $\bar{P}^{\text {theor }}$ in Fig. 12(b) using the Leontovich surface impedance (Decca, López, Fischbach, et al., 2005). As shown in Fig. 12, both theoretical approaches are consistent with the data. Note that the values of theoretical pressures in Fig. 12 are computed at the experimental separations $a_{i}$. As a result, these values are associated with the additional errors $4 \Delta a / a_{i}$ discussed in Sec. III.C.2. They are the primary theoretical 


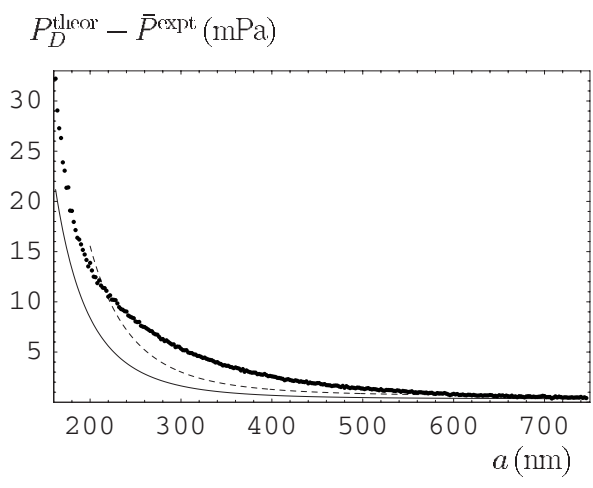

FIG. 13. Comparison of experiment and theory. The difference between theoretical Casimir pressure calculated with the optical data extrapolated using the Drude model and mean exprimental Casimir pressure vs separation. The solid and dashed lines indicate the borders of the $95 \%$ and $99.9 \%$ confidence intervals, respectively.

errors at short separations in this approach to comparing theory with experiment. The respective width of the confidence interval $\left[-\Xi_{P}(a), \Xi_{P}(a)\right]$ is overestimated. The actual agreement of the theoretical approach with data can be characterized by the deviation of the differences $P^{\text {theor }}\left(a_{i}\right)-P^{\operatorname{expt}}\left(a_{i}\right)$ from zero. At the shortest separations this deviation exceeds the total experimental error but (for the theory using the generalized plasmalike permittivity) at $a>300 \mathrm{~nm}$ both are approximately equal. The measure of agreement between experiment and theory given by $\Xi_{P} /\left|\bar{P}^{\text {expt }}\right|$, as defined in Sec. III.C.2, is equal to $1.9 \%$ and $1.8 \%$ at separations $a=162$ and $400 \mathrm{~nm}$, respectively.

In Fig. 13 the same method of comparing theory with experiment is applied to the theoretical approach based on the Drude model. The solid and dashed lines indicate the limits of the $95 \%$ and $99.9 \%$ confidence intervals, respectively (Decca et al., 2007b). The differences $P_{D}^{\text {theor }}\left(a_{i}\right)-P^{\operatorname{expt}}\left(a_{i}\right)$ are shown as dots. As shown in the figure, the theoretical approach using the extrapolation of the optical data to low frequencies by means of the Drude model is experimentally excluded at a $95 \%$ confidence level for the entire measurement range from 162 to $746 \mathrm{~nm}$. Also note that the differences $P_{D}^{\text {theor }}\left(a_{i}\right)$ $-P^{\operatorname{expt}}\left(a_{i}\right)$ are outside the $99.9 \%$ confidence interval at separations from 210 to $620 \mathrm{~nm}$. Finally Decca et al. (2007a, 2007b) concluded that their experiments cannot be reconciled with the Drude model approach to the thermal Casimir force. They also remarked that the experiments with the micromachined torsional oscillator are not of sufficient precision to measure the small thermal effect between the two metal bodies at separations of a few hundred nanometers, as predicted by the generalized plasmalike model. The proposed experiments capable of this goal are considered in Sec. IV.D.

The experimental data by Decca et al. (2007a, 2007b) were also compared with the theoretical results obtained using the modification of the transverse magnetic reflection coefficient in accordance with Eq. (40) (Dalvit and

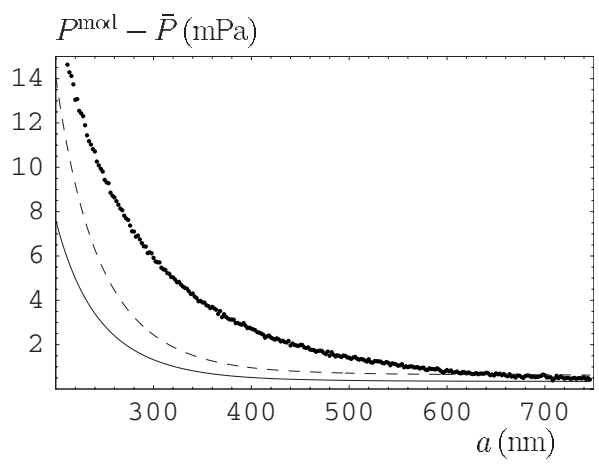

FIG. 14. Comparison of experiment and theory. The difference between theoretical Casimir pressures using the effect of charge screening for the transverse magnetic mode and mean experimental data vs separation is shown as dots. The solid and dashed lines indicate the borders of the $95 \%$ and $99.9 \%$ confidence intervals, respectively.

Lamoreaux, 2008). A charge carrier density of $n \approx 5.9$ $\times 10^{22} \mathrm{~cm}^{-3}$ at $T=300 \mathrm{~K}$ and the Thomas-Fermi screening length were used in the computations for Au. In Fig. 14 the differences between the computed theoretical Casimir pressures and the experimental data by Decca et al. (2007a, 2007b) are shown as dots. As shown, the data exclude the theoretical approach using the modification (40) at a 95\% confidence level within the entire measurement region from 160 to $750 \mathrm{~nm}$ and at a $99.9 \%$ confidence level within the measurement region from 160 to $640 \mathrm{~nm}$. Note that for metals the same theoretical results, as in the approach by Dalvit and Lamoreaux (2008), are obtained in the approaches by Pitaevskii (2008) and Svetovoy (2008). Thus, the last two approaches are also inconsistent with the experimental data by Decca et al. (2007a, 2007b).

Another experiment performed by means of the micromechanical torsional oscillator was the test of corrections to the PFA (Krause et al., 2007). Taking these corrections into account, the Casimir force between a sphere of radius $R$ and a plate can be presented in the form (Scardicchio and Jaffe, 2006)

$$
F_{s}(a, R)=2 \pi R E(a)\left[1+\beta a / R+O\left(a^{2} / R^{2}\right)\right] .
$$

Here $E(a)$ is the Casimir energy per unit area of two parallel plates and $\beta$ is a dimensionless parameter characterizing the lowest-order deviation from the PFA. The constraints on the parameter $\beta$ in Eq. (87) can be obtained from the static measurements of the Casimir force. In these measurements the separation distance between the sphere and the plate is not varied harmonically and the force $F_{s}(a, R)$ is a directly measured quantity.

Dynamic measurements of the Casimir pressure described above in this section are more precise than the static ones. Substituting the Casimir force (87) into the right-hand side of Eq. (85), one obtains the following expression for the effective Casimir pressure (Krause et al., 2007): 


$$
P^{\mathrm{eff}}(a, R)=P(a)\left[1+\tilde{\beta}(a) a / R+O\left(a^{2} / R^{2}\right)\right],
$$

where $P(a)$ is the Casimir pressure between two parallel plates and the dimensionless quantity $\tilde{\beta}(a)$ is given by

$$
\tilde{\beta}(a)=\beta[1-E(a) / a P(a)] .
$$

Note that for ideal metal bodies $\tilde{\beta}(a)=2 \beta / 3=$ const.

To obtain constraints on $\beta$ and $\tilde{\beta}$ a series of both static and dynamic measurements has been performed with Au-coated plate and spheres with radii $R=10.5,31.4$, $52.3,102.8$, and $148.2 \mu \mathrm{m}$. The static measurement of the Casimir force between the sphere and the plate was performed at separations from 160 to $750 \mathrm{~nm}$ in $10 \mathrm{~nm}$ steps. Dynamic determination of the effective Casimir pressure between two parallel plates was done at separations from 164 to $986 \mathrm{~nm}$ with $2 \mathrm{~nm}$ steps. The influences of the effects of the nonzero skin depth and surface roughness on the dominant first-order correction to the PFA were estimated to be of order $10 \%$ and $1 \%$, respectively. Comparison between data and theory at separations $a<300 \mathrm{~nm}$ leads to the result $|\tilde{\beta}(a)|<0.4$ at a $95 \%$ confidence level. In the same separation region, $|\beta|<0.6$ was obtained (Krause et al., 2007). These constraints are compatible with the exact results for a cylinder-plate configuration but are not in agreement with the extrapolations made for a sphere above a plate (see the discussion in Sec. II.B).

\section{Other experiments on the Casimir force between metal bodies}

\section{Torsion pendulum experiment}

Chronologically, the first experiment, in the more recent series of the Casimir force measurements, was performed by Lamoreaux (1997). While this experiment rekindled interest in the investigation of the Casimir force and stimulated further development of the field, the results obtained contain several uncertainties. The Casimir force between a Au-coated spherical lens and a flat plate was measured using a torsion pendulum. A lens with a radius $R=11.3 \pm 0.1 \mathrm{~cm}$ [later corrected to $12.5 \pm 0.3 \mathrm{~cm}$ (Lamoreaux, 1998)] was mounted on a piezo stack and a plate on an arm of the torsion balance in vacuum. The other arm of the torsion balance formed the center electrode of a dual parallel plate capacitor. The positions of this arm and consequently the angle of the torsion pendulum were controlled by the application of voltages to the plates of the dual capacitor. The Casimir force between the plate and the lens would result in a torque, leading to a change in the angle of the torsion balance. This change results in changes of the capacitances. Then compensating voltages were applied to these capacitances to counteract the change in the angle of the torsion balance. These compensating voltages were a measure of the Casimir force.

The calibration of the measurement system was done electrostatically. When the lens and plate surfaces were grounded, a "shockingly large" (Lamoreaux, 1997) re- sidual potential difference $V=430 \mathrm{mV}$ was measured. The corresponding large electrostatic force was compensated with application of voltage to the lens. However, there appears to have been a large residual electrostatic force even after this compensation, which was determined only by fitting to the total force including the Casimir force above $1 \mu \mathrm{m}$. "Typically, the Casimir force had magnitude of at least $20 \%$ of the electrical force at the point of closest approach" (Lamoreaux, 1997). The uncertainty in the measurement of absolute separations $\Delta a$ "was normally less than $0.1 \mu \mathrm{m}$ " (Lamoreaux, 1997).

Data were compared with theory for the ideal metal lens and plate. The conclusion reached that there is an agreement at the level of $5 \%$ in the $0.6-6 \mu \mathrm{m}$ range (Lamoreaux, 1997) was found to be incompatible with the magnitude of the thermal correction to the Casimir force (Bordag et al., 1998). Thus, at separations of 4, 5, and $6 \mu \mathrm{m}$ the thermal corrections are $86 \%, 129 \%$, and $174 \%$ of the zero-temperature force, respectively. The data, however, were found to be "not of sufficient accuracy to demonstrate the finite temperature correction" (Lamoreaux, 1997). From this it follows that the agreement of the data with the theory at the level of $5-10 \%$ may exist only at separations of about $1 \mu \mathrm{m}$. Here the thermal correction is relatively small, whereas the larger corrections due to the skin depth and surface roughness have the opposite sign and partially compensate each other. Keeping in mind that theoretical forces calculated at experimental separations are burdened with an additional error of about $3 \Delta a / a \approx 30 \%$ at $a=1 \mu \mathrm{m}$ [see Sec. III.C.2 and Iannuzzi, Gelfand, et al. (2004)], the errors in the differences $F^{\operatorname{expt}}\left(a_{i}\right)-F^{\text {theor }}\left(a_{i}\right)$, as shown in Fig. 4, bottom (Lamoreaux, 1997), are significantly underestimated.

The results of this experiment at about $1 \mu \mathrm{m}$ separation were used (Torgerson and Lamoreaux, 2004; Lamoreaux, 2005) to exclude the theoretical approach to the thermal Casimir force which uses the Drude model at low frequencies. The latter predicts $-19 \%$ thermal correction at $a=1 \mu \mathrm{m}$ (to be compared with a $1.2 \%$ thermal correction in the case of ideal metals) which was not experimentally observed. The conclusion that the Drude model approach is inconsistent with data is in agreement with the same conclusion obtained at a high confidence level in the much more precise experiments of Decca, Fischbach, et al. (2003), Decca et al. (2004, 2007a, 2007b), and Decca, López, Fischbach, et al. (2005) (see Sec. IV.B).

\section{Micromechanical devices actuated by the Casimir force}

Chan et al. (2001a) used a micromachined torsional device actuated by the Casimir force. A more advanced version of this device shown in Fig. 9 was later used in precise measurements of the Casimir force (see Sec. IV.B). When the sphere in Fig. 9 was moved closer to the plate in a vacuum with a pressure of less than $1 \mathrm{mtorr}$, the Casimir force, acting on the plate, tilted it about its central axis toward the sphere. Thus, vacuum oscillations of the electromagnetic field led to the mechanical 


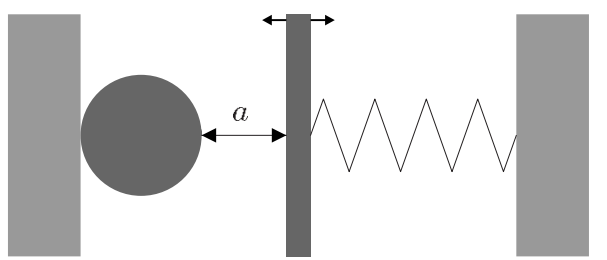

FIG. 15. Schematic diagram for the model of oscillator actuated by the Casimir force.

motion of the plate demonstrating the first micromechanical device driven by the Casimir force. Using a micromachined oscillator, Chan et al. (2001a) measured the Casimir force between a Au-coated polystyrene sphere and a plate. Comparison of the measurement data with the ideal metal Casimir result demonstrated again the influence of skin depth and surface roughness on the Casimir force between real material bodies [previously demonstrated by Mohideen and Roy (1998) and Harris et al. (2000); see Figs. 5 and 6].

A similar device was used to investigate the influence of the Casimir force on the oscillatory behavior of micromachines (Chan et al., 2001b). The simple model of the Casimir oscillator is shown in Fig. 15. It consists of a movable metallic plate which is subject to both the restoring force of a spring and the Casimir force from the interaction with a fixed metallic sphere. The force from the spring is linear in the movement of the plate $\Delta a$, whereas the Casimir force is nonlinear in $\Delta a$. The potential energy of this microdevice possesses local and global minima separated by the potential barrier. The Casimir force changes the resonant frequency of oscillations around the local minimum and makes the oscillations anharmonic (Chan et al., 2001b). These properties may be useful in future micromechanical systems driven by the Casimir force.

\section{The experiment using the parallel plate configuration}

The only experiment in the recent series of Casimir force measurements which uses the original configuration of two plane plates was performed by Bressi et al. (2002). A Si cantilever and a thick plate rigidly connected to a frame (source) both covered with a Cr layer with adjustable separation distance between them were used as two plates. The coarse separation distance was adjusted with a dc motor and fine tuning was achieved using a linear piezoelectric transducer attached to the frame. Calibration was performed using the electrostatic force. As a result, the error in absolute separation was found to be $\Delta a=35 \mathrm{~nm}$. Small oscillations induced on the source by the application of a sinusoidal voltage to the piezo induce oscillations of the cantilever through the Casimir force. Thus, this experiment is dynamical like those considered in Sec. IV.B. The motion of the resonator placed in the vacuum was detected by means of a fiber optic interferometer. After subtracting the electrostatic forces, the residual frequency shift is given by

$$
\Delta \nu_{\text {expt }}^{2}(a)=\nu^{2}-\nu_{0}^{2}=-\alpha \partial P(a) / \partial a
$$

Here $\nu_{0}=138.275 \mathrm{~Hz}$ is the natural frequency in the absence of the Casimir pressure $P$ and $\alpha=S / 4 \pi^{2} m_{\text {eff }}$ $\approx 0.0479 \mathrm{~m}^{2} / \mathrm{kg}$ ( $S$ is the area of a capacitor formed by the plates and $m_{\text {eff }}$ is the effective mass).

Since the measurement is a dynamic one, the directly measured quantity is the frequency shift (90) arising from the effect of the Casimir force. This frequency shift is related to the gradient of the Casimir pressure using Eq. (90). Thus, though this experiment uses the configuration of two parallel plates, it is an indirect measurement of the gradient of the Casimir pressure between the plates, similarly to the experiments using the sphereplate configuration in the dynamic regime for the same purpose (see Sec. IV.B). Bressi et al. (2002) did not aim to restore the Casimir pressure from the pressure gradient. Instead, they fitted the experimental data $\Delta \nu_{\text {expt }}^{2}(a)$ to $\Delta \nu_{\text {theor }}^{2}(a)$ computed from the theoretical dependence of the Casimir pressure between two ideal metal plates $-K_{C} / a^{4}$ with a free parameter $K_{C}$. The best fit resulted in $K_{C}=(1.22 \pm 0.18) \times 10^{-27} \mathrm{~N} \mathrm{~m}^{2}$. This was compared with the exact Casimir coefficient for ideal metal plates in Eq. (2), $K_{C}=\pi^{2} \hbar c / 240=1.3 \times 10^{-27} \mathrm{~N} \mathrm{~m}^{2}$. The conclusion was drawn that the related force coefficient is determined at the $15 \%$ precision level (Bressi et al., 2002).

From the point of view of a general method for the comparison of experiment with theory, which was developed after this experiment was performed, it would be reasonable not to use any fit but to compare $\Delta \nu_{\text {expt }}^{2}(a)$ with the exact expression for $\Delta \nu_{\text {theor }}^{2}(a)$ with no adjustable parameters such as $K_{C}$. In Fig. 16(a) the results of such comparison are presented where the experimental data are shown as crosses and the solid line shows $\Delta \nu_{\text {theor }}^{2}$ computed from the exact expression for the Casimir pressure between two ideal metal plates, as given in Eq. (2). As shown, at separations below $1 \mu \mathrm{m}$ the experimental crosses only touch the solid line. This can be explained by the role of the nonzero skin depth. If instead of $P(a)=P_{0}(a)$ one uses the Casimir pressure with the first and second order corrections due to the nonzero skin depth (Bezerra, Klimchitskaya, and Mostepanenko, 2000),

$$
P(a)=-\frac{\pi^{2} \hbar c}{240 a^{4}}\left(1-\frac{16}{3} \frac{c}{\omega_{p} a}+24 \frac{c^{2}}{\omega_{p}^{2} a^{2}}\right)
$$

the agreement with data improves. As an illustration, $\Delta \nu_{\text {theor }}^{2}$ is recalculated using the theoretical Casimir pressure (91) with the $\omega_{p} \approx 13 \mathrm{eV}$ for $\mathrm{Cr}$. The results are shown as the solid line in Fig. 16(b). It is seen that the Casimir pressure taking the skin depth into account is in much better agreement with the data than the ideal metal Casimir pressure. Suggestions on how to improve the sensitivity of this experiment are discussed in Sec. IV.D. 

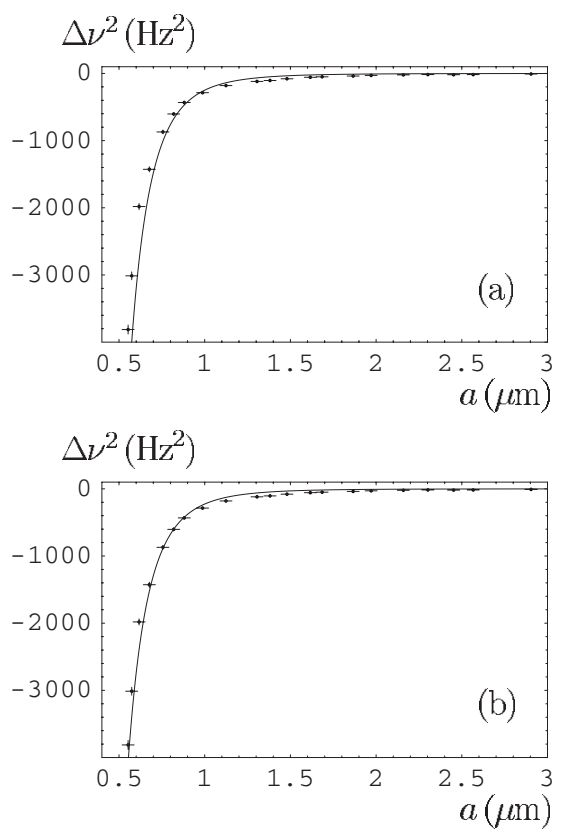

FIG. 16. Comparison of experiment and theory. The measured frequency shift vs separation together with the absolute errors in separation and $\Delta \nu^{2}$ is shown as crosses. Solid lines indicate the theoretical frequency shift calculated for (a) ideal metal plates and (b) real metals with inclusion of the skin-depth correction.

\section{The Casimir force between thin metallic films}

Lisanti et al. (2005) reported the observation of the skin-depth effect on the Casimir force between metallic surfaces. The Casimir force between a thick plate and a $100-\mu$ m-radius polystyrene sphere coated with metallic films of different thicknesses was measured. The sphere was positioned above a micromachined torsional balance. The Casimir attraction between the sphere and the top plate of the balance induced a rotation angle which was measured as a function of the separation between the surfaces. Without the indication of errors and confidence levels, it was reported that the Casimir attraction between the metallic plate and the sphere with coatings thinner than the skin depth is smaller than that of the same plate and a sphere with thick metal coating. Physically, this is in fact the same effect as is demonstrated in Figs. 5 and 6 in Sec. IV.A where the Casimir forces acting between ideal and real metals were compared with corrections based on the plasma frequency (finite conductivity corrections). Ideal metals are better reflectors and the magnitude of the Casimir force between them is larger than between real metals. In a similar manner thick real metal films are better reflectors than thin real metal films.

Lisanti et al. (2005) compared their experimental results with the Lifshitz theory at zero temperature adapted for the description of layered structures (Zhou and Spruch, 1995; Klimchitskaya et al., 2000; Tomaš, 2002). It was shown that the experimental forces obtained for films of thickness smaller than the skin depth have smaller magnitudes than those computed for such films using the Lifshitz theory. This result is not surprising because for films of small thickness the effects of spatial dispersion should be taken into account which are not included in the Lifshitz theory (Klimchitskaya et al., 2000).

\section{Dynamic measurement using an atomic force microscope}

Jourdan et al. (2009) recently performed a measurement of the Casimir force between a Au-coated sphere of $R=20 \mu \mathrm{m}$ radius and a plate using an AFM. No direct sphere-plate contact was used to determine absolute separation distances between the surfaces. The AFM cantilever with the glued sphere was considered as a harmonic oscillator with the natural resonant frequency $\omega_{0}=2 \pi \times 50182 \mathrm{rad} / \mathrm{s}$ which is modified by the effect of the Casimir force. In these two aspects (direct contact between the two surfaces was avoided and the dymanic measurement mode was used) the experimental approach resembles the one used by Decca, López, Fischbach, et al. (2005) and Decca et al. (2007a, 2007b) in the measurements of the Casimir pressure by means of a micromechanical torsional oscillator. The uncertainty in the absolute separations $\Delta a=2 \mathrm{~nm}$ was, however, more than three times larger than in experiments with a micromachined oscillator (see Sec. IV.B). The experimental data for the force gradient were compared with the Lifshitz theory at zero temperature. The dielectric permittivity along the imaginary frequency axis was found using the complete optical data (Palik, 1985) extrapolated to zero frequency by means of the Drude model with $\omega_{p}=9.0 \mathrm{eV}$ and $\gamma=0.035 \mathrm{eV}$ (Lambrecht and Reynaud, 2000). The correction due to surface roughness was not taken into account. The discrepancy between the force gradient measurements and the results of the theoretical computations described above was found to be within $3 \%$ of the theoretical force at separations between 100 and $200 \mathrm{~nm}$. The measured Casimir force gradient was also compared with that computed using ideal metal surfaces and a deviation was reported. On this basis it was concluded that the experimental data demonstrate again the finite conductivity (skin-depth) effects on the Casimir force.

\section{Ambient Casimir force measurements}

In this recent period two other ambient (open to air) experiments measuring the Casimir force were reported. Both did not use a vacuum environment and reported the presence of water layers on the interacting surfaces. The first of these was the experiment by Ederth (2000), where the force was measured between two cylindrical template stripped gold surfaces with $0.4 \mathrm{~nm}$ roughness, in a distance range from 20 to $100 \mathrm{~nm}$. The $200 \mathrm{~nm}$ gold films were fixed to $10 \mathrm{~mm}$ radius silica cylinders using a "soft glue." In addition, a hydrocarbon layer of hexadecanethiol was applied to the interacting surfaces. It was noted that this top hydrocarbon layer was necessary to preserve the purity of the gold surface in the ambient environment. This hydrocarbon organic layer prevented a direct measurement of the electrostatic forces or an 
independent determination of the surface separation. Also the presence of the hydrocarbon layer means that the experiment cannot be strictly classified as that between two gold surfaces. One of the cylinders was mounted on a piezo and the other on bimorph deflection sensor. The charge produced by the bimorph in response to the deflection induced by a force on the interacting surfaces was measured by an electrometer amplifier. The soft glue led to a deformation which was estimated to be 18-20 nm for glue thicknesses used. To compensate for this deformation the two measured force-distance curves were shifted, one by $9 \mathrm{~nm}$ and the other by $12 \mathrm{~nm}$ to overlap the calculations. They reported that "it is not possible to establish with certainty" the validity of the displacement due to the deformation and that it "diminishes the strength of the measurement as a test of the Casimir force and also precludes a quantitative assessment of the agreement between theory and experiment."

A second experiment done in an ambient environment using the AFM for separation distances between 12 and $200 \mathrm{~nm}$ was recently reported (van Zwol et al., 2008). Here a Au-coated sphere of $R=20 \mu \mathrm{m}$ radius was fixed to a gold-coated AFM cantilever. The Au-coated plate was mounted on the piezo. Both the sphere and plate were coated with $100 \mathrm{~nm}$ of Au. The optical properties of the Au-coated plate were measured with an ellipsometer in the wavelength region from $137 \mathrm{~nm}$ to $33 \mu \mathrm{m}$ and fitted to obtain the plasma frequency of $7.9 \pm 0.2 \mathrm{eV}$ and a relaxation frequency of $0.048 \pm 0.005 \mathrm{eV}$ since the finite conductivity corrections for the separation range considered are large. The roughness or the water layer was not taken into account in the fit. The errors in the cantilever spring constant and the deflection coefficient were reported to be $4 \%$ and $3 \%$, respectively, which together were reported to lead to errors of $4-10 \%$. The calibration errors were reported to lead to an overall error of about $5-35 \%$. The electrostatically measured contact potential $10 \pm 10 \mathrm{mV}$ was reported to lead to a $10 \%$ error. They reported that they were not able to independently determine the separation on contact of the two surfaces due to the stiff cantilevers employed. Based on the roughness they estimated a $1 \mathrm{~nm}$ error in the contact separation "leading therefore to a $28 \%$ relative error at the smallest separations." However, a general $10 \%$ agreement with the theory was reported below $100 \mathrm{~nm}$ separation. Given the ambient nature of the experiment, "typically a few nanometers," water layers on both surfaces were present but not treated in the theoretical comparison or systematic errors. Repeating the experiments in a vacuum environment should allow for a more definitive comparison.

\section{Related measurements}

An interesting preliminary test of the Lifshitz theory for three-layer systems is the measurement of the attractive Casimir force between a Au-coated sphere and a plate immersed in ethanol using an AFM (Munday and
Capasso, 2007). The experimental data obtained were compared with the Lifshitz theory taking into account the frequency dependence of the dielectric functions of $\mathrm{Au}$ and ethanol and the correction due to surface roughness. Consistency of the obtained data with Lifshitz's theory was claimed although at separations below $50 \mathrm{~nm}$ a disagreement has been observed which increases with the decrease of separation. However, as discussed (Geyer, Klimchitskaya, Mohideen, and Mostepanenko, 2008), the theoretical computations of the Casimir force between the smooth Au surfaces separated by ethanol done according to the method provided by Munday and Capasso (2007) [i.e., by the use of the Kramers-Kronig relations and tabulated optical data (Palik, 1985)] lead to a discrepancy up to $25 \%$ with respect to the reported theoretical results. The latter can be reproduced if one uses at all imaginary frequencies the Drude dielectric function (35) for both the sphere and plate materials. A second drawback is that the effect of the residual potential difference between the sphere and the plate was calculated incorrectly and significantly underestimated by a factor of 590. Finally, the possible interaction between the double layer formed in liquids due to salt impurities, which would decrease the electrostatic force, was not taken into account without any justification. The resulting electrostatic force is of the same magnitude as the Casimir force to be measured. All this makes the interpretation of this experiment uncertain (Geyer, Klimchitskaya, Mohideen, and Mostepanenko, 2008). In the reply (Munday and Capasso, 2008) it was recognized that the original paper (Munday and Capasso, 2007) did in fact use the Drude model. It was also recognized that the equation originally used to estimate the residual electrostatic force "is not strictly correct" and that salt contaminants exist even in the purest solutions leading to the screening of electrostatic interaction. In a later work (Munday et al., 2008) the effect of electrostatic forces and Debye screening on the measurement of the Casimir force in fluids was further investigated. The electrostatic force with account of Debye screening was calculated as outlined by Geyer, Klimchitskaya, Mohideen, and Mostepanenko (2008). The influence of the concentration of salt impurities on the Debye screening was investigated. No measurable change in the force with or without grounding of the sphere was reported. The Casimir force was measured 51 times with the standard deviation which varies from 130 to $90 \mathrm{pN}$ when separation increases from 30 to $80 \mathrm{~nm}$ (the variances are less by a factor of $\sqrt{51}$ ). As a result, the relative random experimental error of the Casimir force measurements in this experiment determined at a $67 \%$ confidence level is $7 \%$ at $a=30 \mathrm{~nm}$ and increases to $60 \%$ at $a=80 \mathrm{~nm}$. Bearing in mind that for $a<30 \mathrm{~nm}$ they recognized deviations between the theory and the measurement data, this experiment can be considered as only a qualitative demonstration of the Casimir force in fluids.

Another experiment used an adaptive holographic interferometer to measure periodical nonlinear deformations of a thin pellicle caused by an oscillating Casimir force due to a spherical lens (Petrov et al., 2006). Both 
test bodies were coated with a thin $\mathrm{Al}$ film and placed in a vacuum chamber. The lens was mounted on a vibrating piezodriver. As a result, the oscillations of the lens position led to a periodic modulation of the Casimir force. The experimental data were found to be in qualitative agreement with the theory based on ideal metal boundaries at separation distances of a few hundred nanometers. Corrections due to the finite skin depth and surface roughness were not provided. Also the use of $\mathrm{Al}$ whose surface undergoes rapid oxidation even in relatively high vacuum adds uncertainty to the results of this experiment. However, the new measurement technique used may be promising for future measurements of the Casimir force.

\section{Future prospects to measure the thermal Casimir force}

As described in Secs. IV.A-IV.C, the measurements of the Casimir force between macroscopic bodies performed to date were not of sufficient precision to measure the magnitude of the thermal effect. The experiments using the micromachined oscillator (Sec. IV.B) possess the highest experimental precision at separations below $1 \mu \mathrm{m}$. They have been used to exclude thermal effects, as predicted by models using the Drude model at low frequencies. However, thermal effects predicted by the generalized plasmalike model at short separations remain below the experimental sensitivity. In this respect, large separation measurements of the Casimir force would be of great interest. At separations of a few micrometers the thermal regime is reached where the Casimir free energy is entirely of thermal origin. Calculations using the plasma model or the generalized plasmalike model result in Eq. (34). The Drude model approach to the thermal Casimir force leads to only one-half of the result for ideal metal plates [Eq. (34) with $\left.\omega_{p} \rightarrow \infty\right]$. Thus, large-separation measurements of the Casimir force would bring direct information to bear on the magnitude of the thermal effect between macroscopic bodies (such a measurement in the atomplate configuration has been performed already; see Sec. VI.A).

In Sec. IV.C.1 it was shown that the experiment using the torsion pendulum within the region from 0.6 to $6 \mu \mathrm{m}$ is in fact uncertain at separations above $2 \mu \mathrm{m}$ where the thermal effects begin to make a substantial contribution. An analysis (Lamoreaux and Buttler, 2005) shows that the torsion pendulum technique has the potential to measure the Casimir force between a plate and a spherical lens at $a=4 \mu \mathrm{m}$ with a relative error of $10 \%$. Bearing in mind that at $a=4 \mu \mathrm{m}$ the thermal correction contributes as much as $86 \%$ of the zerotemperature Casimir force, such an experiment, if successfully performed, holds great promise.

There is a proposal aimed at measuring the Casimir force in the cylinder-plate configuration at separations around $3 \mu \mathrm{m}$ (Brown-Hayes et al., 2005). This geometry can be considered as a compromise between the twoparallel-plate configuration (which is connected with serious experimental difficulties associated with the parallelism of the plates) and a sphere above a plate. In addition, as discussed in Sec. II.B, the exact solution for the ideal metal cylinder-plate configuration has been obtained recently. This gives the possibility to determine the accuracy of the PFA and to apply it to real materials with high reliability. Finally it was concluded that using the dynamic measuring scheme it is possible to measure the Casimir force between a plate and a cylinder at separations of about $3 \mu \mathrm{m}$ with a precision of a few percent (Brown-Hayes et al., 2005).

Another proposal suggests to use a highly sensitive torsion balance in the separation range from 1 to $10 \mu \mathrm{m}$ to measure the Casimir force in the configuration of two parallel plates (Lambrecht et al., 2005). The construction of the balance is similar to that used in the Eötvos-type experiments aimed to test the equivalence principle. It is planned to measure the thermal Casimir force with an accuracy of a few percent and to discriminate between different theoretical approaches discussed.

One more experiment exploiting the two-parallelplate configuration is proposed at separations larger than a few micrometers (Antonini et al., 2006). The experimental scheme is based on the use of a Michelsontype interferometer and the dynamic technique with one oscillating plate. Calibrations show that a force of 5 $\times 10^{-11} \mathrm{~N}$ can be measured in this setup with the relative error from about $10 \%$ to $20 \%$. This would be sufficient to measure the thermal effect at a separation of $5 \mu \mathrm{m}$ (Antonini et al., 2006).

The thermal effect in the Casimir force can be measured at short separations below $1 \mu \mathrm{m}$ if the difference in the thermal forces $\Delta F$ at different temperatures rather than the absolute value of the thermal Casimir force is measured (Chen et al., 2003). For real metals, this difference of the thermal Casimir forces (in contrast with the relative thermal correction) does not increase but decreases with increasing separation distance. This allows the observation of the thermal effect on the Casimir force at small separations of about $0.5 \mu \mathrm{m}$ where the relative thermal correction, as predicted by the generalized plasmalike model, is rather small. Preliminary estimation shows that with a sphere of $R=2 \mathrm{~mm}$ radius attached to a cantilever of an AFM the measurable changes of the force amplitude of order $10^{-13} \mathrm{~N}$ are achievable from a $50 \mathrm{~K}$ change in the temperature. Such a temperature difference can be obtained by the illumination of the sphere and plate surfaces with laser pulses of $10^{-2} \mathrm{~s}$ duration (Chen, Mohideen, and Milonni, 2004). The idea of exploiting difference force measurements to probe the thermal Casimir effect in superconducting cavities was proposed by Bimonte (2008).

From the above discussion it is clear that experimental investigations of thermal effects in the Casimir force appear feasible in the near future. 


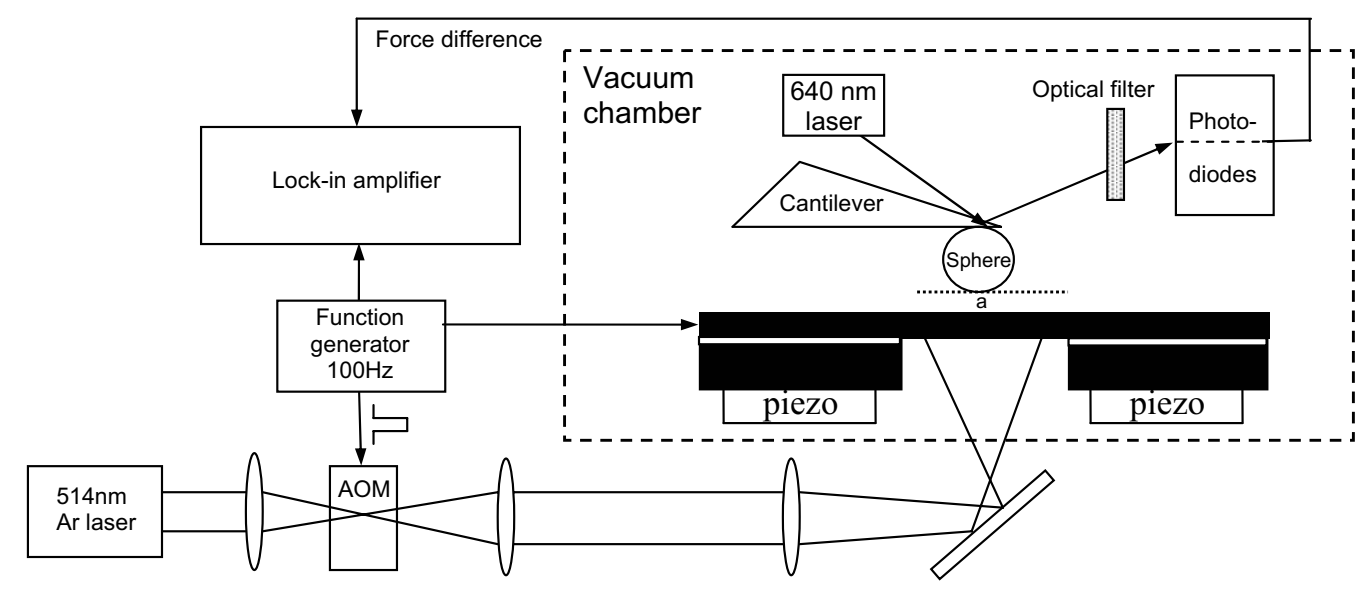

FIG. 17. Schematic of the experimental setup on measuring the difference Casimir force between Au sphere and Si plate illuminated with laser pulses.

\section{CASIMIR FORCE BETWEEN A METALLIC SPHERE AND A SEMICONDUCTOR PLATE}

\section{A. Motivation for use of semiconductors}

A vital issue in many applications of the Casimir effect is how to control the magnitude of the force by changing the parameters of the system. A natural method for this control is to change the material properties of the interacting bodies. Arnold et al. (1979) made an attempt to modify the van der Waals and Casimir forces between semiconductors with light. Attractive forces were measured between a glass lens and a $\mathrm{Si}$ plate and also between a glass lens coated with amorphous Si and a Si plate. The glass lens, however, is an insulator and therefore the electric forces, such as due to work function potential differences, could not be controlled. This might explain why Arnold et al. (1979) found no force change occurred on illumination at separations below $350 \mathrm{~nm}$, where it should have been most pronounced. One more attempt to modify the Casimir force was made by Iannuzzi, Lisanti, and Capasso (2004) when measuring the Casimir force acting between a plate and a sphere coated with a hydrogen-switchable mirror that becomes transparent upon hydrogenation. Despite expectations, no significant decrease of the Casimir force owing to the increased transparency was observed. The negative result is explained by the Lifshitz theory, which requires a change of the reflectivity properties within a wide range of frequencies in order to markedly affect the magnitude of the Casimir force. This requirement is not satisfied by hydrogenation.

The appropriate materials for the control, modification, and fine tuning of the Casimir force are semiconductors. The reflectivity properties of semiconductor surfaces can be changed in a wide frequency range by changing the carrier density through the variation of temperature, using different kinds of doping, or, alternatively, via illumination of the surface with laser light. At the same time, semiconductor surfaces with reasonably high conductivity avoid accumulation of excess charges and, thus, preserve the advantage of metals for Casimir force measurements. In addition, as semiconductors are the basic fabrication materials for nanotechnology, the use of semiconductor surfaces for the control of the Casimir force will lead to many applications.

\section{B. Optically modulated Casimir force}

The modification of the Casimir force through the radiation induced change in the carrier density was first demonstrated by Chen et al. (2007a, 2007b). A highvacuum-based AFM was employed to measure the change in the Casimir force between a Au-coated sphere and a Si membrane in the presence and in the absence of incident light. An oil-free vacuum chamber with a pressure of around $2 \times 10^{-7}$ torr was used. A polystyrene sphere of diameter $2 R=197.8 \pm 0.3 \mu \mathrm{m}$ coated with a $\mathrm{Au}$ layer of $82 \pm 2 \mathrm{~nm}$ thickness was mounted at the tip of a $320 \mu \mathrm{m}$ conductive cantilever (the general scheme of the experiment is shown in Fig. 17). A specially fabricated $\mathrm{Si}$ membrane [see Chen et al. (2007b) for preparation details] was mounted on top of the piezo which is used to change the separation distance $a$ between the sphere and the membrane from contact to $6 \mu \mathrm{m}$. The excitation of the carriers in the $\mathrm{Si}$ membrane was done with 5 -ms-wide light pulses (50\% duty cycle). These pulses were obtained from a cw Ar ion laser light at $514 \mathrm{~nm}$ wavelength modulated at a frequency of $100 \mathrm{~Hz}$ using an acousto-optic modulator (AOM). The laser pulses were focused on the bottom surface of the Si membrane. The Gaussian width of the focused beam on the membrane was measured to be $w=0.23 \pm 0.01 \mathrm{~mm}$. The resulting modification of the Casimir force in response to the carrier excitation was measured with a lock-in amplifier (see Fig. 17). The same function generator signal used to generate the Ar laser pulses was also used as a reference for the lock-in amplifier.

The illumination of $\mathrm{Si}$ has to be done such that very little if any light-impinges on the sphere, as this would lead to a light-induced force from the photon pressure. As the $\mathrm{Si}$ membrane is illuminated from the bottom, care should be taken that the fraction of light transmit- 
ted through the membrane is negligibly small. The optical absorption depth for $\mathrm{Si}$ at a wavelength of $514 \mathrm{~nm}$ is $1 \mu \mathrm{m}$. The measured thickness of the membrane used was $4.0 \pm 0.3 \mu \mathrm{m}$. The calculations presented showed that for the membrane thicknesses used, the force on the sphere due to photon pressure varies from $2.7 \%$ to $8.7 \%$ of the difference of dispersion forces to be measured when separation increases from 100 to $200 \mathrm{~nm}$.

The calibration of the setup, the determination of the cantilever deflection coefficient, and the average separation on contact between the test bodies are performed as in earlier experiments with metal test bodies [see Sec. IV.A and Harris et al. (2000); Chen, Mohideen, et al. (2006); Chen et al. (2007b); Chiu et al. (2008)]. The deflection coefficient was $m=137.2 \pm 0.6 \mathrm{~nm}$ per unit deflection signal. The difference in the value of $m$ from previous measurements (see Sec. IV.A) is due to the use of the $514 \mathrm{~nm}$ optical filter (see Fig. 17) which reduces the cantilever deflection signal. This filter was used to prevent the interference of the $514 \mathrm{~nm}$ excitation light with the cantilever deflection signal from the $640 \mathrm{~nm}$ laser (see Fig. 17). The separation distance on contact was $a_{0}=97 \mathrm{~nm}$. The uncertainty in the quantity $a_{0}+m S_{\text {def }}$ was $1 \mathrm{~nm}$. This leads to practically the same error $\Delta a$ $=1 \mathrm{~nm}$ in the absolute separations (83) because the error in piezocalibration is negligibly small. An independent measurement of the lifetime of the carriers excited in the Si membrane by pulses from the Ar laser was performed using a noninvasive optical pump-probe technique (Nagai and Kuwata-Gonokami, 2002; Sabbah and Riffe, 2002).

In the experiment under consideration it is not sufficient to determine the residual potential difference $V_{0}$ between the sphere and the membrane as described in Sec. IV.A. In the presence of a pulse train the values of the residual potential difference can be different: $V_{0}^{l}$ and $V_{0}$ during the bright and dark phases of a laser pulse train, respectively. Both values are generally different from the residual potential difference determined in the absence of pulse train. In the measurement procedure, the voltages $V^{l}$ and $V$ are applied to the membrane during the bright and dark phases, respectively. The difference in the total force (electric and Casimir) for the states with and without carrier excitation was measured. Using Eq. (82), this difference can be written in the form

$$
\Delta F_{\text {tot }}(a)=X(a)\left[\left(V^{l}-V_{0}^{l}\right)^{2}-\left(V-V_{0}\right)^{2}\right]+\Delta F(a) .
$$

Here $\Delta F(a)$ is the difference of the Casimir forces for the states with and without light. By keeping $V=$ const and changing $V^{l}$, the parabolic dependence of $\Delta F_{\text {tot }}$ on $V^{l}$ was measured. The value of $V^{l}$ where this function reaches a maximum [recall that $X(a)<0$ in Eq. (82)] is $V_{0}^{l}$. Then by keeping $V^{l}=$ const and changing $V$, the parabolic dependence of $\Delta F_{\text {tot }}$ on $V$ was measured, which allows one to find $V_{0}$. Both procedures were repeated at different separations and the values $V_{0}^{l}=-0.303 \pm 0.002 \mathrm{~V}$ and $V_{0}=-0.225 \pm 0.002 \mathrm{~V}$ were found to be independent of separation in the range from 100 to $500 \mathrm{~nm}$. These values were shown to change only
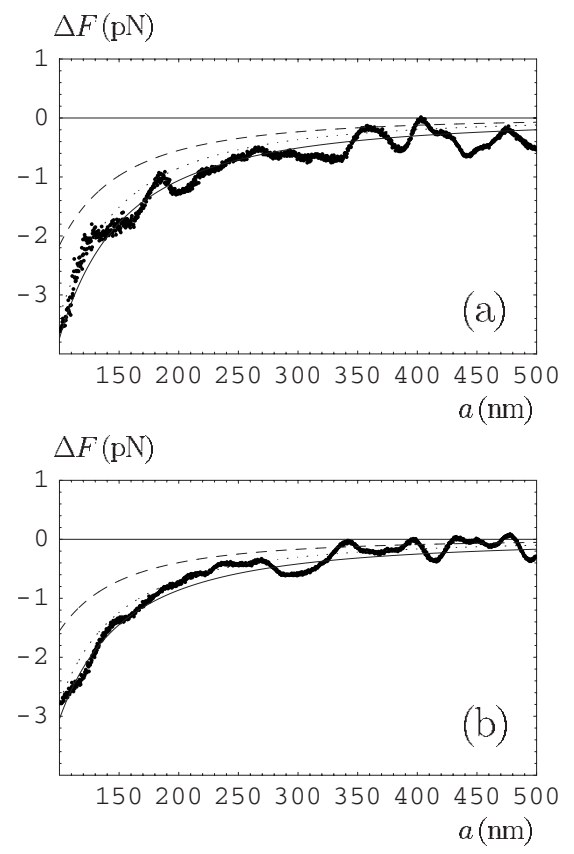

FIG. 18. The difference of the Casimir forces in the presence and in the absence of light vs separation for different absorbed powers (a) 9.3 and (b) $4.7 \mathrm{~mW}$. The measured differences $\left\langle F^{\text {expt }}\right\rangle$ are shown as dots. See text for the description of theoretical solid, dotted, and dashed lines computed using the different approaches.

within the resolution error. It was stated that the independence of the residual potential difference of separation is a basic and necessary condition for every Casimir force measurement. The dependence of $V_{0}$ of separation indicates the presence of electrostatic surface impurities, space charge effects, and/or electrostatic inhomogeneities on the sphere or plate surface (Chiu et al., 2008). The small change in the residual potential difference between the sphere and the membrane in the presence and absence of excitation light is primarily due to the screening of surface states by few of the optically excited electrons and holes. The value of this difference of around $78 \mathrm{mV}$ is equal to the change in band bending at the surface. It is consistent with the fact that almost flat bands are obtained at the surface with the surface passivation technique used for the preparation of the $\mathrm{Si}$ membrane (Chen et al., 2007b).

Then other voltages $\left(V^{l}, V\right)$ were applied to the $\mathrm{Si}$ membrane and the difference in the total force $\Delta F_{\text {tot }}$ was measured as a function of separation within the interval from 100 to $500 \mathrm{~nm}$. From these measurement results, the difference in the Casimir force $\Delta F^{\operatorname{expt}}(a)$ was determined from Eq. (92). This procedure was repeated with some number of pairs $(J)$ of different applied voltages $\left(V^{l}, V\right)$ and at each separation the mean value $\left\langle\Delta F^{\operatorname{expt}}(a)\right\rangle$ was found. The measurements were performed for different absorbed laser powers: $P^{\text {eff }}=9.3 \mathrm{~mW} \quad(J=31)$, $8.5 \mathrm{~mW}(J=41)$, and $4.7 \mathrm{~mW}(J=33)$, corresponding to the incident powers of $15.0,13.7$, and $7.6 \mathrm{~mW}$, respectively. The experimental data for $P^{\mathrm{eff}}=9.3$ and $4.7 \mathrm{~mW}$ are shown by dots in Figs. 18(a) and 18(b), respectively. 


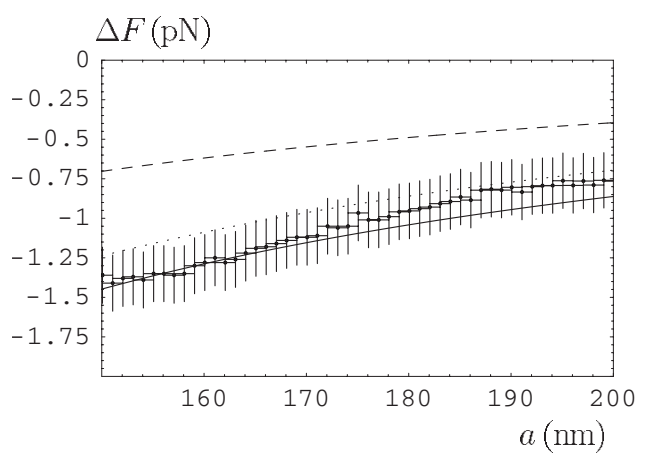

FIG. 19. Comparison of experiment and theory. The experimental differences in the Casimir force with their experimental errors are shown as crosses (the absorbed power is $4.7 \mathrm{~mW}$ ). The solid and dotted lines represent the theoretical differences computed at $T=300 \mathrm{~K}$ using the model with a finite static permittivity of high-resistivity Si but different models for $\mathrm{Si}$ in the presence of light (see text for further discussion). The dashed line represents the theoretical differences computed at the same temperature including the dc conductivity.

As expected, the magnitude of the Casimir force difference has the largest values at the shortest separations and decreases with decrease of the separation. It also decreases with decrease of the absorbed laser power.

The analysis of the experimental errors performed in line with Sec. III.C.1 (Chen et al., 2007b) shows that the total experimental error in this experiment is equal to the random one. The relative experimental errors at a $95 \%$ confidence level vary from $10 \%$ to $20 \%$ at a separation $a=100 \mathrm{~nm}$ and from $25 \%$ to $33 \%$ at a separation $a=180 \mathrm{~nm}$ for different absorbed laser powers. As an example, in Fig. 19 the experimental data are shown with their absolute errors calculated at a $95 \%$ confidence level for the absorbed power $4.7 \mathrm{~mW}$ in the separation region from 150 to $200 \mathrm{~nm}$ [each third dot from Fig. 18(b) is shown]. Thus, the modulation of the dispersion force with light is demonstrated by Chen et al. (2007a, 2007b) at a high reliability and confidence.

For the comparison of experimental results with theory, the difference of dispersion forces between a sphere and a plate was calculated according to the first equality in Eq. (16) with the free energy given by the Lifshitz formula (3) (Chen et al., 2007a, 2007b). The surface roughness was taken into account using geometrical averaging (75). It was shown that the contribution from the roughness correction to the calculated value of $\Delta F(a)$ is small. At the shortest separation $a=100 \mathrm{~nm}$ it is only $1.2 \%$ and decreases to $0.5 \%$ at $a=150 \mathrm{~nm}$. The calculations were done at the laboratory temperature $T=300 \mathrm{~K}$.

Both cases of the dielectric permittivity of the highresistivity $(10 \Omega \mathrm{cm}) \mathrm{Si}$ in the absence of light were considered, i.e., the inclusion or the neglect of the dc conductivity (see Sec. II.D.2). The dielectric permittivity of the dielectric $\mathrm{Si}$ (no light and the de conductivity is disregarded) along the imaginary frequency axis was found from the tabulated optical data (Palik, 1985). It is shown in Fig. 20 as the long-dashed line. Here $\varepsilon_{0}=11.66$. Note

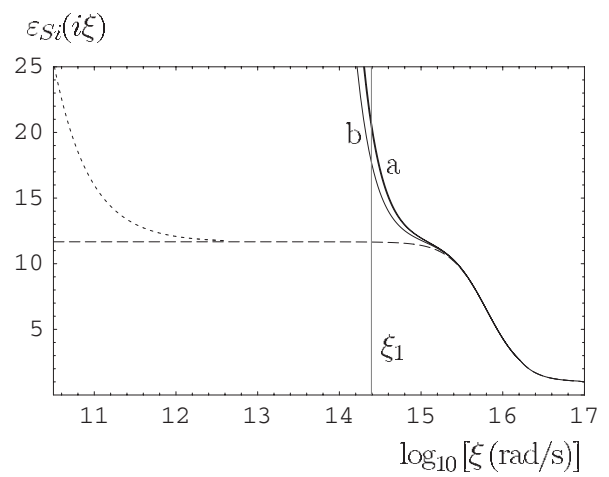

FIG. 20. The dielectric permittivity of the Si membrane along the imaginary frequency axis for Si with different concentration of charge carriers (see text for further discussion).

that the use of the analytic approximation for $\varepsilon_{\mathrm{Si}}$ (Inui, 2003 ) leads to about $10 \%$ error in the magnitudes of the Casimir force (Chen, Mohideen, et al., 2006) and, thus, is not suitable for the comparison with precise measurements. The dielectric permittivity of the high-resistivity Si with de conductivity included (the charge carrier density $\tilde{n} \approx 5 \times 10^{14} \mathrm{~cm}^{-3}$ ) is shown in Fig. 20 by the shortdashed line.

On irradiation of the Si membrane with light, an equilibrium value of the carrier density is rapidly established during a period of time much shorter than the duration of a laser pulse (note that the lifetimes were independently measured). This allows one to assume that there is an equilibrium concentration of pairs (electrons and holes) when the light is incident. In this case the dielectric permittivity along the imaginary frequency axis is commonly represented in the form [see, e.g., Palik (1985), Vogel et al. (1992), and Inui (2004, 2006)]

$$
\varepsilon_{\mathrm{Si}}^{(l)}(i \xi)=\varepsilon_{\mathrm{Si}}(i \xi)+\omega_{p(e)}^{2} / \xi\left[\xi+\gamma_{(e)}\right]+\omega_{p(p)}^{2} / \xi\left[\xi+\gamma_{(p)}\right]
$$

where $\omega_{p(e, p)}$ and $\gamma_{(e, p)}$ are the plasma frequencies and the relaxation parameters of electrons and holes, respectively $\left[\gamma_{(e)} \approx 1.8 \times 10^{13} \mathrm{rad} / \mathrm{s}, \gamma_{(p)} \approx 5.0 \times 10^{12} \mathrm{rad} / \mathrm{s}\right.$ (Vogel et al., 1992); the values of the plasma frequencies are given below]. To avoid violation of the thermal equilibrium arising from the use of the Drude model (see Sec. II.D.1), it was suggested (Mostepanenko and Geyer, 2008) that the influence of free charge carriers in metaltype semiconductors shold be included by means of the generalized plasmalike model (58). This rule should also be applied to all doped semiconductors with dopant concentration above critical. Then, instead of Eq. (93), the dielectric permittivity is given by

$$
\varepsilon_{\mathrm{Si}}^{(l)}(i \xi)=\varepsilon_{\mathrm{Si}}(i \xi)+\omega_{p(e)}^{2} / \xi^{2}+\omega_{p(p)}^{2} / \xi^{2} .
$$

The plasma frequency can be calculated from

$$
\omega_{p(e, p)}=\left(n e^{2} / m_{e, p}^{*} \epsilon_{0}\right)^{1 / 2},
$$

where the effective masses are $m_{p}^{*}=0.2063 m$ and $m_{e}^{*}$ $=0.2588 \mathrm{~m}$, and $n$ is the concentration of charge carriers (Vogel et al., 1992). The value of $n$ for the different ab- 
sorbed powers can be calculated with the knowledge that at equilibrium the number of charge carrier pairs created per unit time per unit volume is equal to the recombination rate of pairs per unit volume. This leads to (Chen et al., 2007b)

$$
n=4 P_{w}^{\mathrm{eff}} \tau / \hbar \omega d \pi w,
$$

where $P_{w}^{\text {eff }}=0.393 P^{\text {eff }}$ is the power in the central part of the Gaussian beam focused on the membrane (see above) and $\tau$ is the measured lifetime of the charge carriers. For the absorbed power $P^{\text {eff }}=9.3 \mathrm{~mW}$ [Fig. 18(a)] it follows from Eqs. (96) and (95) that $n_{a}=(2.1 \pm 0.4)$ $\times 10^{19} \mathrm{~cm}^{-3}, \quad \omega_{p(e)}=(5.1 \pm 0.5) \times 10^{14} \mathrm{rad} / \mathrm{s}, \quad$ and $\omega_{p(p)}$ $=(5.7 \pm 0.6) \times 10^{14} \mathrm{rad} / \mathrm{s}$ (Chen et al., 2007b). Here and below all errors are found at a $95 \%$ confidence level. For $P^{\text {eff }}=4.7 \mathrm{~mW}$ [Fig. 18(b)] the same parameters are $n_{b}$ $=(1.4 \pm 0.3) \times 10^{19} \mathrm{~cm}^{-3}, \omega_{p(e)}=(4.1 \pm 0.4) \times 10^{14} \mathrm{rad} / \mathrm{s}$, and $\omega_{p(p)}=(4.6 \pm 0.4) \times 10^{14} \mathrm{rad} / \mathrm{s}$. [Note that Chen et al. (2007b) contains two misprints corrected here.] The corresponding dielectric permittivities are shown in Fig. 20 with the solid lines labeled $a$ and $b$. It should be remarked that the dielectric permittivities (93) and (94) almost overlap at all nonzero Matsubara frequencies.

The dielectric permittivity of $\mathrm{Au}$ along the imaginary frequency axis is presented in Fig. 1. For the dielectric permittivity of $\mathrm{Si}$ in the dark phase and the bright phase when Eq. (93) is used, the reflection coefficient is $r_{\mathrm{TE}}^{\mathrm{Si}}\left(0, k_{\perp}\right)=0$. Thus, the calculation results do not depend on whether the solid or the dashed line in Fig. 1 for $\varepsilon_{\mathrm{Au}}(i \xi)$ is used. If in the presence of light $\varepsilon_{\mathrm{Si}}^{(l)}(i \xi)$ is given by Eq. (94), then the Au dielectric permittivity in the framework of the generalized plasmalike model should be used (the solid line in Fig. 1).

The results of the numerical computations of the difference Casimir force $\Delta F^{\text {theor }}(a)$ between rough surfaces with the dielectric permittivity (94) in the presence of light and by neglecting dc conductivity in the absence of light are shown by the solid lines in Fig. 18 (for different absorbed powers). The results of the analogous calculations with the dielectric permittivity (93) in the presence of light are shown in Fig. 18 with the dotted lines. As seen from Fig. 18, both the solid and dotted lines are in good agreement with the experimental data shown by dots. Thus, the experiment under consideration does not allow one to discriminate between the dielectric permittivities (93) and (94) used above for the description of charge carriers in metal-type semiconductors. This is also seen in Fig. 21 where the dots present the values of the differences between $\Delta F^{\text {theor }}(a)$ and $\left\langle\Delta F^{\operatorname{expt}}(a)\right\rangle$ for the absorbed power of $4.7 \mathrm{~mW}$ and the solid lines show the $95 \%$ confidence interval calculated as described in Sec. III.C.2 (Chen et al., 2007b; Mostepanenko and Geyer, 2008). Dots labeled 1 in Figs. 21(a) and 21(b) are obtained with $\varepsilon_{\mathrm{Si}}(i \xi)$ in the dark phase but with the dielectric permittivities (93) or (94) in the bright phase, respectively. It can be observed that the dots labeled 1 are inside the confidence intervals in both Figs. 21(a) and 21(b). To distinguish between the two models using the dielectric permittivities (93) and (94) of metal-type
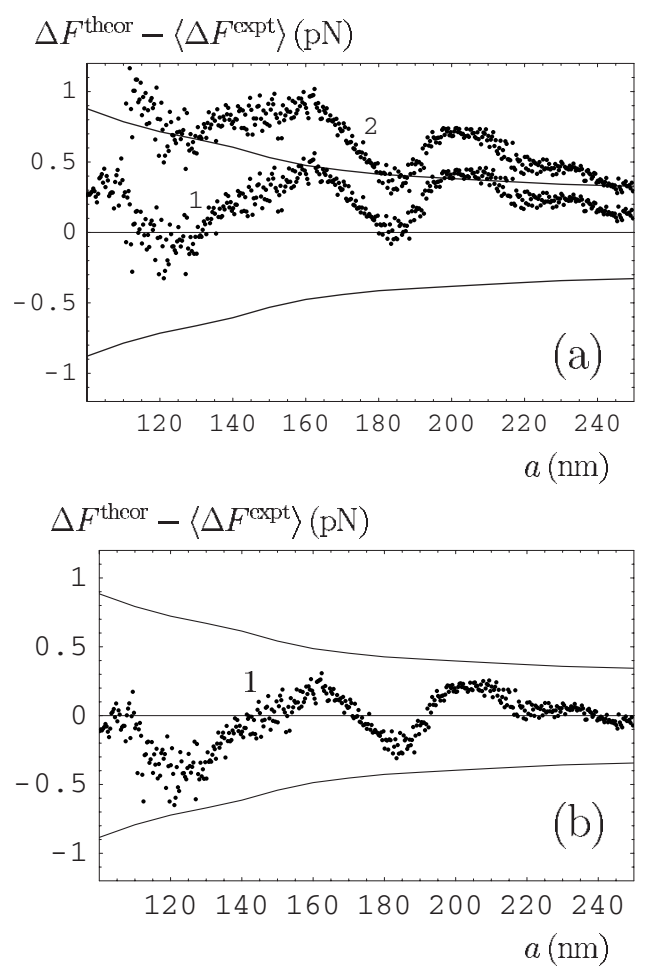

FIG. 21. Comparison of experiment and theory. Theoretical minus experimental differences of the Casimir force vs separation are shown as dots. In the absence of the laser pulse the theoretical results for dots labeled 1 are computed with a finite static dielectric permittivity of high resistivity $\mathrm{Si}$ and for dots labeled 2 taking the dc conductivity of high resistivity Si into account. When the laser pulse is on, the charge carriers are described by (a) the Drude and (b) the plasma model. Solid lines show the $95 \%$ confidence interval.

semiconductors more precise experiments are required (see Sec. V.D.2).

The calculation of the theoretical differences $\Delta F^{\text {theor }}(a)$ using (in the absence of light) the dielectric permittivity with the inclusion of dc conductivity leads to the results presented by the dashed lines in Figs. 18 and 19. As shown, this theoretical prediction is excluded by the data. The same can also be observed in Fig. 21(a) where the dots labeled 2 present the differences between the theoretical results calculated with the small dc conductivity of the Si membrane included in the absence of light and the experimental data. Almost all dots labeled 2 are outside the $95 \%$ confidence interval. This means that a model which includes the de conductivity of the high-resistivity $\mathrm{Si}$ is excluded by the experimental data at a $95 \%$ confidence level within the separation region from 100 to $200 \mathrm{~nm}$.

The results of this experiment were applied (Klimchitskaya, Mohideen, and Mostepanenko, 2008) to test the modification of the transverse magnetic reflection coefficient, as given by Eq. (40) (Dalvit and Lamoreaux, 2008) and Eq. (53) with the standard contributions of all nonzero Matsubara frequencies (Pitaevskii, 2008). The experimental data for the difference of the Casimir forces in the presence and absence of laser light are 

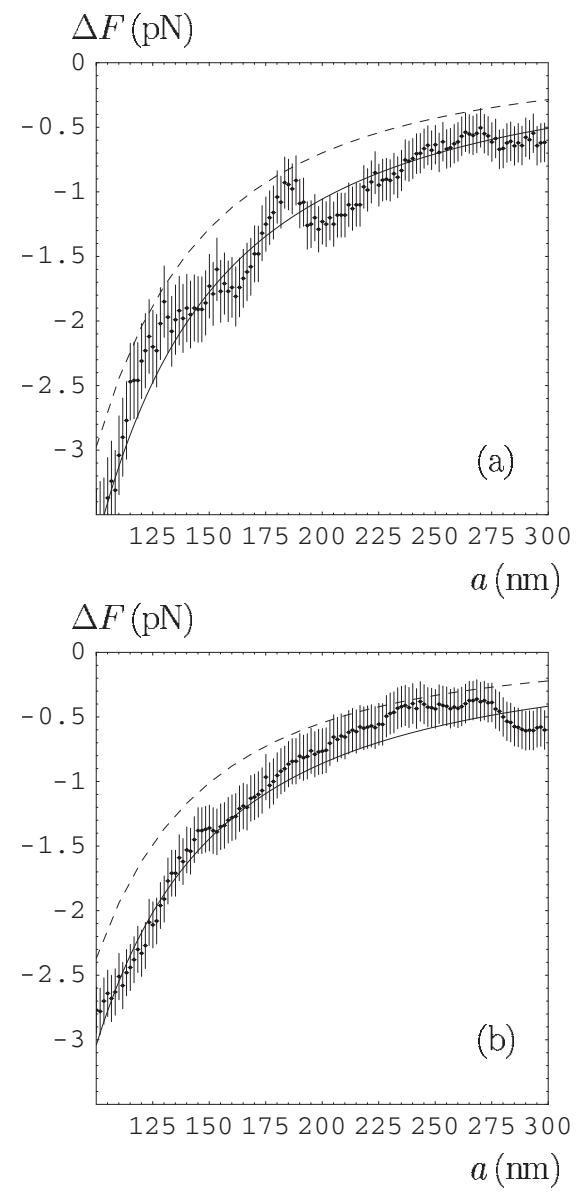

FIG. 22. Difference of the Casimir forces between a Aucoated sphere and Si plate in the presence and absence of laser light on the plate vs separation for the absorbed powers of (a) 9.3 and (b) $4.7 \mathrm{~mW}$. The experimental data are shown as crosses. The solid and dashed lines are computed using the standard Lifshitz theory with the dc conductivity of Si in the dark phase neglected and taking into account the effect of charge screening for the transverse magnetic mode, respectively.

shown in Fig. 22 for the absorbed powers (a) of $9.3 \mathrm{~mW}$ and (b) of $4.7 \mathrm{~mW}$. In contrast to Fig. 19, the experimental errors are presented at a $70 \%$ confidence level. The computational results obtained on the basis of the standard Lifshitz theory with the dc conductivity neglected in the dark phase are shown by the solid lines. The dashed lines are computed using Eq. (40) for the modified TM reflection coefficient at all $x=\xi_{l}$ with concentration of charge carriers $n=\tilde{n}$ and the Debye-Hückel length in the dark phase and $n=2 n_{a}$ or $n=2 n_{b}$ in the presence of light. The computation of the dashed lines was repeated using Eq. (53) for the TM reflection coefficient at zero frequency. At all nonzero Matsubara frequencies, the standard terms of the Lifshitz formula were used. In both cases practically coinciding computational resuts were obtained, shown as the dashed lines in Figs. 22(a) and 22(b). As shown in Figs. 22(a) and 22(b), the experimental data are consistent with the theoretical results computed on the basis of the standard Lifshitz theory with the dc conductivity of dielectric Si neglected in the dark phase (the solid lines). The theoretical results computed using Eq. (40) at all $\xi_{l}$ or Eq. (53) with the standard contributions of all nonzero Matsubara frequencies are excluded by data at a 70\% confidence level (Klimchitskaya, Mohideen, and Mostepanenko, 2008).

According to Svetovoy (2008), the experimental data for the difference Casimir force are equally consistent with the nonlocal approach using the reflection coefficient (53) and the Lifshitz theory with dc conductivity neglected in the dark phase. To prove this, the experimental data of Fig. 22(a) at a 70\% confidence level were used, but the dashed line was replaced with the theoretical band whose width was determined at a $95 \%$ confidence level using the respective uncertainty in charge carrier density $\Delta n=0.4 \times 10^{19} \mathrm{~cm}^{-3}$. Such a mismatched comparison of experiment with theory is irregular. It can be seen that the theoretical bands related to the solid and dashed lines in Fig. 22(a) do not overlap if one uses in computations the uncertainty in charge carriers $\Delta n$ $=0.3 \times 10^{19} \mathrm{~cm}^{-3}$ determined at the same $70 \%$ confidence level as the experimental errors (Mostepanenko et al., 2009).

The experimental results presented here support the conclusion made in Sec. II.D.2. In accordance with this conclusion, one should disregard the role of free charge carriers in all dielectric materials (in particular, in semiconductors with dopant concentration below critical) when calculating dispersion forces in the framework of the Lifshitz theory.

\section{Doped semiconductors with different charge carrier densities \\ 1. $p$-type silicon}

The most important materials used in nanotechnology are semiconductors with conductivity properties ranging from the metallic to the dielectric. As mentioned in Sec. V.A, semiconductors with a relatively high conductivity have an advantage that they avoid accumulation of residual charges but, at the same time, possess a typical dielectric dependence of the dielectric permittivity on frequency within a wide frequency range (see, e.g., Fig. 20). This makes it possible to examine the influence of doping concentration on the Casimir force. Chen et al. (2005), and Chen, Mohideen, et al. (2006) first measured the Casimir force between a Au-coated sphere of the diameter $2 R=202.6 \pm 0.3 \mu \mathrm{m}$ (a Au layer of $105 \mathrm{~nm}$ thickness was used) and $5 \times 10 \mathrm{~mm}^{2}$ single crystal silicon $\mathrm{Si}\langle 100\rangle$ plate. The resistivity of this $p$-type B-doped plate measured using the four-probe technique was $\rho$ $=0.0035 \Omega \mathrm{cm}$.

The Casimir force was measured with an improved version of the setup previously used (Harris et al., 2000) for the two Au test bodies (see Sec. IV.A). The main improvements in the experimental setup were the use of much higher vacuum and the reduction of the uncertainty in the determination of absolute separation $a$ [see Eq. (83)]. As in Sec. V.B, a much higher vacuum (2 $\times 10^{-7}$ torr) is needed to maintain the chemical purity of 


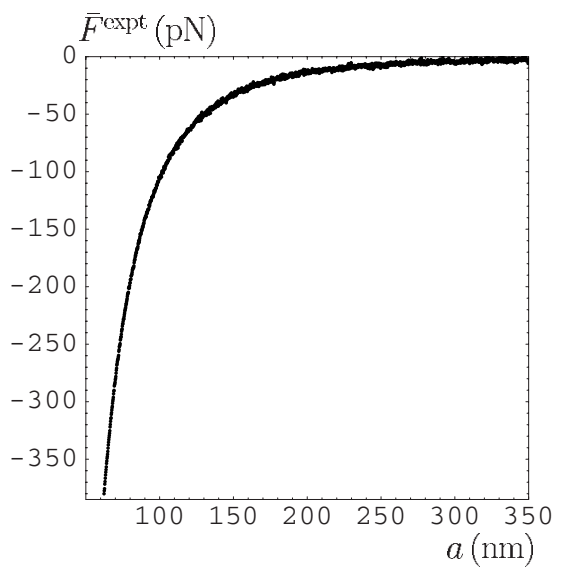

FIG. 23. The mean measured Casimir force vs separation between a Au sphere and B-doped Si plate.

the $\mathrm{Si}$ surface which otherwise oxidizes rapidly to $\mathrm{SiO}_{2}$. The high-vacuum system consists of oil-free mechanical pumps, turbo pumps, and ion pumps. To maintain the lowest pressure during data acquisition, only the ion pump is used. This helps to reduce the influence of mechanical noise. The absolute error in the determination of absolute separation was reduced to $\Delta a=0.8 \mathrm{~nm}$ in comparison to $\Delta a=1 \mathrm{~nm}$ (see Sec. IV.A). This was achieved using a piezo capable of traveling a distance $6 \mu \mathrm{m}$ from initial separation to contact. More details on the improvements are provided by Chiu et al. (2008).

In contrast to $\mathrm{Au}$, the $\mathrm{Si}$ surface is very reactive. Because of this, a special passivation procedure is needed to prepare it for force measurements (Chen, Mohideen, et al., 2006). To characterize the topography of both samples, the Au coating on the sphere and the surface of the Si plate were investigated using an AFM. Images resulting from the surface scan of the Au coating show that the roughness is represented mostly by stochastically distributed distortions of about $8-22 \mathrm{~nm}$ height with $\delta_{\mathrm{st}}^{(1)}=3.446 \mathrm{~nm}$. The surface scan of the Si surface shows much smoother distortions with typical heights from 0.4 to $0.6 \mathrm{~nm}$ and $\delta_{\mathrm{st}}^{(1)}=0.111 \mathrm{~nm}$.

All calibrations and determination of the residual electrostatic force and the separation on contact were done immediately before the Casimir force measurements in the same high-vacuum apparatus (see the description in Sec. IV.A). The force calibration constant was $1.440 \pm 0.007 \mathrm{nN}$ per unit cantilever deflection signal, and the residual potential difference was $V_{0}=-0.114 \pm 0.002 \mathrm{~V}$.

In Fig. 23, the mean measured Casimir force is presented (average of 65 measurements). The variance of this mean is found to be approximately the same over the entire measurement range $62.33 \leqslant a \leqslant 349.97 \mathrm{~nm}$ and equal to $1.5 \mathrm{pN}$. Thus, the random experimental error at a $95 \%$ confidence level is $3.0 \mathrm{pN}$. The systematic error in this experiment, determined at a $95 \%$ confidence level (in line with Sec. III.C.1), is $1.17 \mathrm{pN}$. The total experimental error, calculated using Eq. (80) at a $95 \%$ confidence level, is $\Delta^{t} F^{\text {expt}} \approx 3.33 \mathrm{pN}$. Thus, the relative ex-

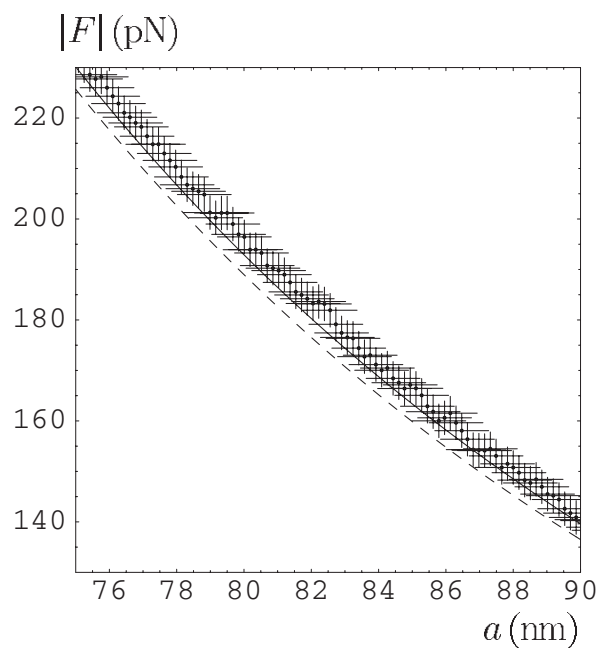

FIG. 24. Comparison of experiment and theory. The magnitude of the experimental Casimir force with the errors shown as crosses vs separation. The solid line shows the theoretical dependence calculated for the sample used in the experiment and the dashed line for a dielectric Si.

perimental error varies from $0.87 \%$ at the shortest separation to $3 \%$ at $a=100 \mathrm{~nm}$ to $64 \%$ at $a=300 \mathrm{~nm}$.

In Fig. 24, the experimental data with their error bars are plotted as crosses for a more narrow separation range from 75 to $90 \mathrm{~nm}$. The solid line in Fig. 24 shows the theoretical results calculated using the Lifshitz theory with the dielectric permittivity (93) [for the $p$-type plate used $\omega_{p(e)}=0, \omega_{p(p)}=7 \times 10^{14} \mathrm{rad} / \mathrm{s}$ (Chen, Mohideen, et al., 2006)]. The dashed line in Fig. 24 presents the calculation results for dielectric Si (the permittivity is shown by the long-dashed line in Fig. 20). As seen from Fig. 24, the solid line is consistent with the experiment whereas the dashed line is inconsistent. Thus, for the Si plate with a rather high doping concentration $\left(n \approx 3 \times 10^{19} \mathrm{~cm}^{-3}\right)$ the presence of free charge carriers markedly influences the Casimir force.

\section{2. $n$-type silicon}

Further investigation of the difference in the Casimir force for samples with different charge-carrier densities was performed for $n$-type Si doped with P (Chen, Klimchitskaya, et al., 2006). The above same high vacuum based AFM was used to measure the Casimir force between a Au-coated sphere of a diameter $2 R$ $=201.8 \pm 0.6 \mu \mathrm{m}$ and two $4 \times 7 \mathrm{~mm}^{2}$ size Si plates with different charge carrier densities placed next to each other. The thickness of the Au coating on the sphere was measured to be $92 \pm 2 \mathrm{~nm}$.

The two Si samples chosen for this experiment were identically polished single crystals of $500 \mu \mathrm{m}$ thickness. The resistivity of the samples was measured using the four-probe technique to be $\rho_{a}=0.43 \Omega \mathrm{cm}$. Thus, the concentration of the charge carriers $n_{a} \approx 1.2 \times 10^{16} \mathrm{~cm}^{-3}$ was well below the critical concentration corresponding to the dielectric-metal transition in $\mathrm{Si}$ doped with $\mathrm{P}$ $\left(n_{\mathrm{cr}} \approx 1.3 \times 10^{18} \mathrm{~cm}^{-3}\right)$. One of these samples was used as 


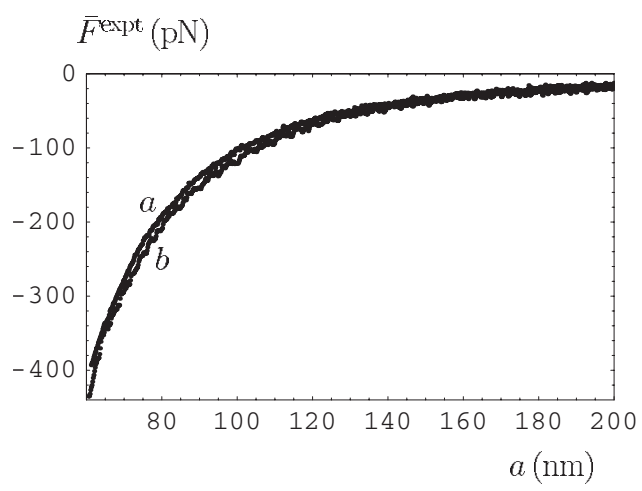

FIG. 25. The mean measured Casimir force vs separation between a Au sphere and two N-doped Si plates of (a) higher and (b) lower resistivities.

the first Si plate in the experiment. The other one was subject to thermal diffusion doping to prepare the second, lower-resistivity plate, with $\rho_{b} \approx 6.7 \times 10^{-4} \Omega \mathrm{cm}$ $\left(n_{b} \approx 3.2 \times 10^{20} \mathrm{~cm}^{-3}\right)$. Both plates of higher and lower resistivity were subjected to a special passivation procedure to prepare their surfaces for the force measurements.

All calibration procedures were done as in the previous measurements (see Sec. IV.A) separately for the samples of higher and lower resistivities. For the sample of higher resistivity, the value of the residual potential difference $V_{0}=-0.341 \pm 0.002 \mathrm{~V}$ was obtained. The cantilever deflection constant was $1.646 \pm 0.004 \mathrm{nN}$ per unit deflection signal and the separation on contact was $a_{0}=32.4 \pm 1.0 \mathrm{~nm}$. For the sample of lower resistivity $V_{0}$ $=-0.337 \pm 0.002 \mathrm{~V}, a_{0}=32.3 \pm 1.0 \mathrm{~nm}$, and the cantilever deflection constant $1.700 \pm 0.004 \mathrm{nN}$ per unit deflection signal was found. Note that the expression for the electric force (82) used in the calibration does not take into account possible influence of screening effects (spacecharge layer at the surface of Si). According to Bingqian et al. (1999), for high-resistivity $n$-type $\mathrm{Si}$ with the concentration of charge carriers of order $10^{16} \mathrm{~cm}^{-3}$, the impact of this layer on the electrostatic force is negligible at separations from $300-400 \mathrm{~nm}$ to $2.5 \mu \mathrm{m}$ where the calibration fit was performed. Obviously, in the case of lower resistivity Si the influence of space-charge layer is even more negligible. These conclusions were experimentally confirmed through the demonstration that both the residual potential difference $V_{0}$ and the separation on contact $a_{0}$ are the same regardless of the separation distance where the calibration fit is performed (Chen, Klimchitskaya, et al., 2006; Chiu et al., 2008).

The Casimir force as a function of the separation was measured for both samples immediately after the calibration procedure was done for each one. The mean values of the measured Casimir force $\bar{F}^{\text {expt }}$ are presented in Fig. 25. Dots labeled $a$ show the results for the sample of higher resistivity (average of 40 measurements). Dots labeled $b$ correspond to the sample of lower resistivity (average of 39 measurements). As seen from Fig. 25, dots labeled $a$ and $b$ are distinct from each other dem-

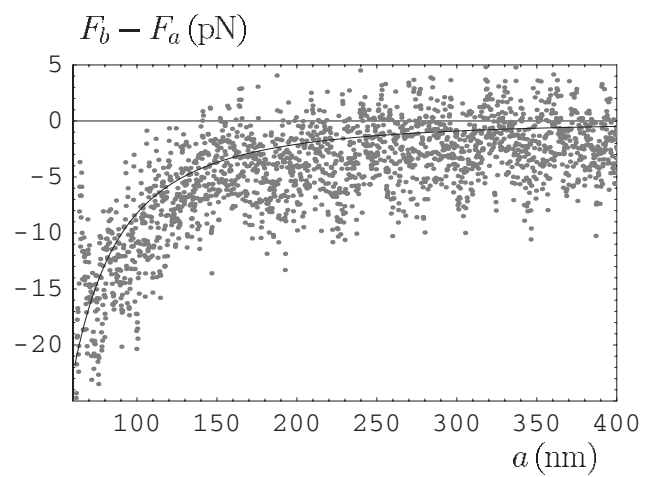

FIG. 26. Comparison of experiment and theory. The differences of the mean measured Casimir forces acting between a $\mathrm{Au}$ sphere and lower and higher resistivity Si samples vs separation are shown as dots. The respective theoretical difference is shown by the solid line.

onstrating the effect of different charge-carrier densities in the two Si plates used.

The error analysis performed, as described in Sec. III.C.1, shows that the total experimental errors determined at a $95 \%$ confidence level are equal to the random ones in each measurement. Thus, $\Delta^{t} F_{a}^{\text {expt }}=8,6$, and $4 \mathrm{pN}$ at $a=61.19$ and $70 \mathrm{~nm}$ and $a \geqslant 80 \mathrm{~nm}$, respectively. The measurement for the sample of lower resistivity is slightly more noisy. Here $\Delta^{t} F_{a}^{\text {expt }}=11,7$, and $5 \mathrm{pN}$ at $a$ $=61.19$ and $70 \mathrm{~nm}$ and $a \geqslant 80 \mathrm{~nm}$. From Fig. 25 it shown that the deviation between the two sets of data is larger than the total experimental error in the separation region from 61.12 to $120 \mathrm{~nm}$.

The force-distance relationship measured for the two Si samples was compared with theory. For the description of the sample with lower resistivity the dielectric permittivity (93) was used [with $\omega_{p(p)}=0, \omega_{p(e)}=2.0$ $\left.\times 10^{15} \mathrm{rad} / \mathrm{s}\right]$. The sample of higher resistivity was described by the dielectric permittivity of a dielectric $\mathrm{Si}$ (the long-dashed line in Fig. 20).

In Fig. 26 the differences of the measured Casimir forces for the plates of the lower and higher resitivity $F_{b}^{\text {expt }}(a)-F_{a}^{\text {expt }}(a)$ versus separation are shown as dots. In the same figure, the difference in the respective theoretically computed Casimir forces is shown by the solid line. As seen from Fig. 26, the experimental and theoretical results as functions of $a$ are in good agreement.

The experiments, described here, demonstrate the possibility of modifying the Casimir force by changing the doping concentration of semiconductor materials.

Note that recently a theoretical investigation of the influence of $\mathrm{Si}$ doping concentration on the Casimir force in the separation region from $1 \mathrm{~nm}$ to $5 \mathrm{~mm}$ was performed using the zero-temperature Lifshitz formula (Pirozhenko and Lambrecht, 2008a). The obtained results, however, are reliable only at separations below $1 \mu \mathrm{m}$ because the use of the zero-temperature Lifshitz formula with the room-temperature parameters at separations above $1 \mu \mathrm{m}$ is physically problematic (Bordag et al., 2001). 


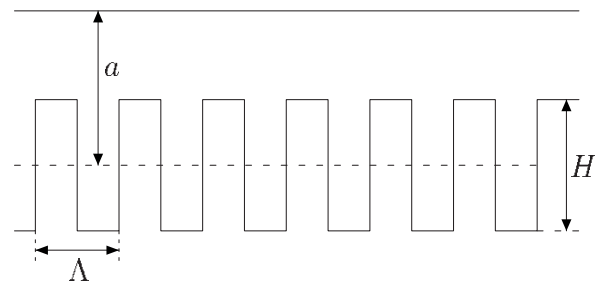

FIG. 27. The periodical uniaxial rectangular corrugations on one of the plates.

\section{Silicon plate with rectangular trenches}

Chan et al. (2008) reported a measurement of the Casimir force between a gold-coated sphere of radius $R$ and a silicon surface that had been structured with nanoscale rectangular corrugations (trenches). Measurements were performed in the dynamic regime using a micromechanical torsional oscillator (see Sec. IV.B). This means that the immediately measured quantity was the change of the resonant frequency of the oscillator which is proportional to the derivative of the Casimir force with respect to separation distance. In accordance with the proximity force approximation given by Eq. (85), this derivative is

$$
F_{s}^{\prime}(a)=-2 \pi R P(a),
$$

where $P(a)$ is the Casimir pressure between one rectangular corrugated plate and one plane plate (see Fig. 27). Note that the experiment was done at room temperature, but the results obtained were compared with theoretical computations at $T=0$.

For an ideal metal case such a configuration was considered by Büscher and Emig (2004) using exact methods presented in Sec. II.B. It was shown that in the limiting case $a \ll \Lambda$ (recall that separation is measured between the zero-corrugation levels of the lower plate and the upper plate) the Casimir pressure for the fractional surface area of solid volume equal to $1 / 2$ is given by

$$
P(a)=-\frac{\pi^{2} \hbar c}{240} \frac{1}{2}\left(\frac{1}{(a-H / 2)^{4}}+\frac{1}{(a+H / 2)^{4}}\right) .
$$

This is in fact the minimum value of the Casimir pressure between the rectangular corrugated and plane plates. Under the condition $a \ll \Lambda$ for ideal metals the same result is obtained by the application of the proximity force approximation or pairwise summation methods (see Sec. III.B.1).

In the opposite limit $a \gg \Lambda$ the exact result is given by (Büscher and Emig, 2004)

$$
P(a)=-\frac{\pi^{2} \hbar c}{240} \frac{1}{(a-H / 2)^{4}},
$$

which is up to a factor of 2 larger in magnitude than the prediction of the proximity force approximation and paiwise summation in Eq. (98). This is the maximum value of the Casimir pressure in the configuration of the rect- angular corrugated and plane plates made of ideal metals.

The trenches were fabricated in $p$-doped silicon (the density of charge carriers $2 \times 10^{18} \mathrm{~cm}^{-3}$ was determined from the dc conductivity which was $0.028 \Omega \mathrm{cm}$ ). Silicon oxide was used as the mask. Deep uv lithography followed by reactive ion etching was used to transfer the pattern. Trenches of approximate depth $1 \mu \mathrm{m}$ were created. Three types of samples were made on the same wafer: sample $A$ with $\Lambda_{A}=1 \mu \mathrm{m}$ period, sample $B$ with $\Lambda_{B}=400 \mathrm{~nm}$ period, and one with a flat surface. The fractional areas of solid volume were $p_{A}=0.478 \pm 0.002$ and $p_{B}=0.510 \pm 0.001$ for samples $A$ and $B$ using a scanning electron microscope. The residual hydrocarbons were removed by oxygen plasma etching and the oxide mask was etched with HF. Samples of size 0.7 $\times 0.7 \mathrm{~mm}^{2}$ were used for the force measurement. The corrugated silicon surface was prepared as done by Chen, Klimchitskaya, et al. (2006) and Chen, Mohideen, et al. (2006) using hydrogen passivation. However, after this the silicon chip was baked to $120^{\circ} \mathrm{C}$ to remove the residual water from the bottom of the trenches, which might impair the passivation layer leading to patch potentials.

The micromachined oscillator used in the measurements consists of a 3.5- $\mu \mathrm{m}$-thick, 500- $\mu \mathrm{m}$-square silicon plate. Unlike in previous experiments with micromachined oscillators (see Sec. IV.B), the spheres were attached to the plate. Two sputter gold coated with thickness of about $400 \mathrm{~nm}$ glass spheres of radius $R=50 \mu \mathrm{m}$ were attached on top of each other to the torsional oscillator at a distance of $b=210 \mu \mathrm{m}$ using conductive epoxy. Two spheres are used to provide a large distance between the corrugated surface and the top of the torsional oscillator. The resonant frequency $\left(\omega_{0}=2 \pi\right.$ $\times 1783 \mathrm{~Hz}$, quality factor $Q=32000)$ was excited by applying voltage on one of the bottom electrodes. The oscillations were detected with additional voltages with an amplitude of $100 \mathrm{mV}$ and a frequency of $2 \pi \times 102 \mathrm{kHz}$ which were applied to measure the capacitance change between the top plate and the bottom electrodes. A phase-locked loop was used to detect the change in the resonant frequency as a function of the distance between the sphere and the corrugated plate.

The measurements were done at a vacuum of $10^{-6}$ torr using a dry roughing pump and a turbo pump. The residual potential difference $V_{0}$ and the initial separation between the surfaces $a_{0}$ were determined and the calibration was done using electrostatic forces. No value of $a_{0}$ or errors in its determination were provided. A residual potential difference $V_{0} \sim-0.43 \mathrm{~V}$ was found between a sphere and a flat Si plate and noted to vary with $3 \mathrm{mV}$ within the separation distance of $100 \mathrm{~nm}$ to $2 \mu \mathrm{m}$. If the same was found for the corrugated Si surfaces this was not mentioned. Voltages between $V_{0}+245 \mathrm{mV}$ and $V_{0}+300 \mathrm{mV}$ were applied and the calibration constant was found to be $628 \pm 5 \mathrm{~m} \mathrm{~N}^{-1} \mathrm{~s}^{-1}$. Since no analytic expression for the electrostatic force is available for the trench geometry a two-dimensional (2D) numerical so- 
lution of the Poisson equation was used to calculate the electrostatic energy between a flat plate and the trench surface. This energy was then converted to a force between a sphere and the trench surface using the PFA.

The Casimir force gradients between the flat plate and samples $A$ and $B$ were measured after the application of compensating voltages to the plates. The main uncertainty in these measurements is reported as that coming from thermomechanical noise with a value of about

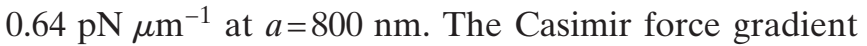
between the flat plate and the gold sphere $F_{\text {flat }}^{\prime}$ was first measured. Good agreement was found with the calculation using the Lifshitz theory. The tabulated data for gold and silicon (Palik, 1985) were used along with the modification corresponding to the carrier density of silicon (Chen, Klimchitskaya, et al., 2006). The roughness correction was taken into account using rms roughnesses of $4 \mathrm{~nm}$ on the sphere and $0.6 \mathrm{~nm}$ on the silicon surface measured using an AFM (see Sec. III.B.1). Next the force gradients $F_{A \text {,expt }}^{\prime}$ and $F_{B \text {,expt }}^{\prime}$ were measured on the corrugated surfaces using the same gold sphere.

For the configurations used by Chan et al. (2008) one can neglect the contribution from the remote bottom parts of trenches. Then the proximity force approximation leads to the following force gradients for samples $A$ and $B$ :

$$
\begin{aligned}
& F_{A, \mathrm{PFA}}^{\prime}(a)=-2 \pi R p_{A} P^{(r)}(a-H / 2), \\
& F_{B, \mathrm{PFA}}^{\prime}(a)=-2 \pi R p_{B} P^{(r)}(a-H / 2),
\end{aligned}
$$

where $P^{(r)}(a)$ is the Casimir pressure between two noncorrugated plates covered with a stochastic roughness calculated using the Lifshitz formula as described in Sec. III.B.1.

To compare the experimental data with theory, Chan et al. (2008) considered the ratios

$$
\rho_{A}=F_{A, \operatorname{expt}}^{\prime} / F_{A, \mathrm{PFA}}^{\prime}, \quad \rho_{B}=F_{B, \text { expt }}^{\prime} / F_{B, \mathrm{PFA}}^{\prime}
$$

It was shown that for sample $A$ there are deviations of $\rho_{A}$ from unity up to $10 \%$ over the measurement range from $a=650$ to $750 \mathrm{~nm}$ exceeding the experimental errors. For sample $B$ there are deviations of $\rho_{B}$ from unity up to $20 \%$ over the same measurement range. This difference in the comparison of the experimental data for samples $A$ and $B$ with the PFA results is natural, as $a / \Lambda_{A}=0.7$ and $a / \Lambda_{B}=1.75$ at a typical separation distance considered $a=700 \mathrm{~nm}$. Thus, for sample $B$ the applicability condition of the PFA, $a / \Lambda \ll 1$, considered in Sec. III.B.1 is violated to a larger extent than for sample A.

The measurements were repeated three times for each sample and consistent results have been observed. The data were also compared to values from the exact calculations for ideal metal boundaries, which were converted to the sphere-trenched plate case using the PFA. However, the measured deviations from PFA, as applied to the rectangular corrugations, were found to be $50 \%$ less than that expected for ideal metals. This discrepancy was reported as quite natural due to the interplay of nonzero skin depth and geometry effects.

Thus, the experiment by Chan et al. (2008) reports the measurement of deviation resulting from geometry for corrugated rectangular trenches of relatively small periods. The depth of the corrugated trenches allowed good comparison to the results obtained using the PFA taking into account only the fractional area of the top surface. Deviations between $10-20 \%$ from the PFA were reported. At present no theoretical computations exist which would allow a definite comparison between experiment and theory for spherical and corrugated surfaces made of real metals at room temperature [at $T$ $=0$ such computations were performed by Lambrecht and Marachevsky (2008)].

\section{E. Future prospects to measure the Casimir force with semiconductor surfaces}

\section{The dielectric-metal transition}

The possibility for the modulation of the Casimir force due to a change of charge carrier density is offered by semiconductor materials that undergo the dielectricmetal transition with the increase of temperature. From a fundamental point of view, the modulation of the Casimir force due to the phase transitions of different kinds offers one more precision test of the role of conductivity and optical properties in the Lifshitz theory of the Casimir force.

An experiment was proposed (Castillo-Garza et al., 2007) to measure the change of the Casimir force acting between a Au-coated sphere and a vanadium dioxide $\left(\mathrm{VO}_{2}\right)$ film deposited on a sapphire substrate which undergoes the dielectric-metal transition with the increase of temperature. It has been known that $\mathrm{VO}_{2}$ crystals and thin films undergo an abrupt transition from semiconducting monoclinic phase at room temperature to a metallic tetragonal phase at $68{ }^{\circ} \mathrm{C}$ (Zylbersztejn and Mott, 1975; Soltani et al., 2004; Suh et al., 2004). The phase transition causes the resistivity of the sample to decrease by a factor of $10^{4}$ from 10 to $10^{-3} \Omega \mathrm{cm}$. In addition, the optical transmission for a wide region of wavelengths extending from $1 \mu \mathrm{m}$ to greater than $10 \mu \mathrm{m}$ decreases by more than a factor of 10-100.

The increase of temperature necessary for the phase transition can be induced by laser light (Soltani et al., 2004; Suh et al., 2004). Thus, a setup similar to the one employed in the demonstration of optically modulated dispersion forces (see Sec. V.B) can be used. In the initial stage of the experimental work, the procedures of film fabrication and their heating were investigated (Castillo-Garza et al., 2007). The preliminary theoretical results are also obtained (Castillo-Garza et al., 2007; Pirozhenko and Lambrecht, 2008a) based on the Lifshitz theory and optical data for $\mathrm{VO}_{2}$ films (Verleur et al., 1968). Calculations of the difference Casimir force between a $\mathrm{Au}$ sphere and $\mathrm{VO}_{2}$ film on sapphire substrate after and before the phase transition showed that the 


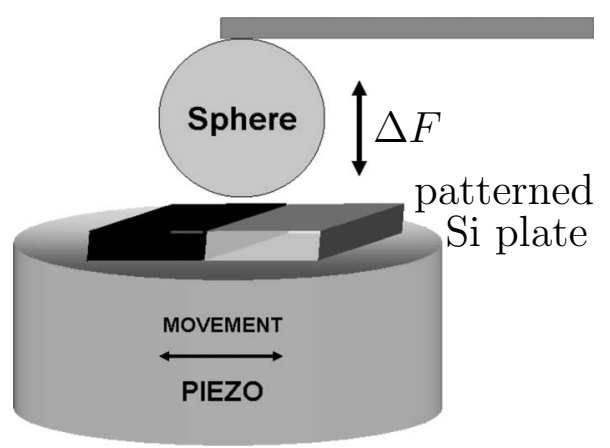

FIG. 28. Schematic diagram of the experimental setup for the measurement of the difference Casimir force between a Aucoated sphere and patterned Si plate.

proposed experiment has much promise for the understanding of the role of free charge carriers in the Lifshitz theory of dispersion forces.

Interesting results can also be obtained when investigating the change of the Casimir free energy in the phase transition of a metal to the superconducting state. The variation of the Casimir free energy during this transition is small (Mostepanenko and Trunov, 1997). Nevertheless, the magnitude of this variation can be comparable to the condensation energy of a semiconducting film and causes a measurable increase in the value of the critical magnetic field (Bimonte, Calloni, Esposito, Milano, and Rosa, 2005; Bimonte, Calloni, Esposito, and Rosa, 2005).

\section{Casimir forces between a sphere and a plate with patterned geometry}

The difference force measurements are very sensitive to relatively small variations of the Casimir force (see Sec. V.B). Recently an experimental scheme was proposed (Castillo-Garza et al., 2007) which promises a record sensitivity to a force difference at the level of 1 $\mathrm{fN}$. The patterned Si plate with two sections of different doping concentrations (see Fig. 28) is mounted on a piezo below a Au-coated sphere attached to the cantilever of an AFM. The piezo oscillates in the horizontal direction causing the flexing of the cantilever in response to the Casimir force above different regions of the plate. Thus, the sphere is subject to the difference Casimir force which can be measured using the static and dynamic techniques. The patterned plate is composed of a single crystal of $\mathrm{Si}$ specifically fabricated to have adjacent sections of two different charge carrier densities. A special procedure was developed for the preparation of the Si sample with the two sections having different conductivities (Castillo-Garza et al., 2007) where the $p$ - and $n$-type dopants can be used (B and $\mathrm{P}$, respectively). Sharp transition boundaries between the two sections of the Si plate of width, below $200 \mathrm{~nm}$ can be achieved. Identically prepared but unpatterned samples can be used to measure the properties which are needed for the theoretical computations (Hall probes for measuring the charge carrier concentration, a four-probe technique for measuring conductivity). The measurement of the difference Casimir force is planned as follows. The Si plate is positioned such that the boundary is below the vertical diameter of the sphere (see Fig. 28). The distance between the sphere and Si plate $a$ is kept fixed and the $\mathrm{Si}$ plate oscillates in the horizontal direction using the piezo such that the sphere crosses the boundary in the perpendicular direction during each oscillation [a similar approach was exploited (Decca, López, Chan, et al., 2005) for constraining new forces from the oscillations of the Au-coated sphere above two dissimilar metals, Au and Ge]. The Casimir force on the sphere changes as the sphere crosses the boundary. This change corresponds to the differential force,

$$
\Delta F(a)=F_{\tilde{n}}(a)-F_{n}(a),
$$

equal to the difference of the Casimir forces due to the different charge carrier densities $\tilde{n}$ and $n$, respectively. This causes a difference in the deflection of the cantilever. In order to reduce random noise by averaging, the periodic horizontal movement of the plate will be of an angular frequency $\Omega \sim 0.1 \mathrm{~Hz}$. The amplitude of plate oscillations is limited by the piezocharacteristics but can be of order $100 \mu \mathrm{m}$, much larger than the typical width of the transition region equal to $200 \mathrm{~nm}$.

The proposed experiment holds promise for the investigation of the possible variation of the Casimir force in the dielectric-metal transition in semiconductors with the increase of doping concentration (Klimchitskaya and Geyer, 2008). It has the potential to distinguish between the two models of the dielectric permittivity of semiconductors with the concentration of charge carriers above the critical [see Eqs. (93) and (94) in Sec. V.B].

\section{Pulsating Casimir force}

At present, a consensus has been reached that applications of the Casimir force in the design, fabrication, and actuation of micromechanical and nanomechanical devices are very promising. When the characteristic sizes of a device shrink below $1 \mu \mathrm{m}$, the Casimir force becomes larger than typical electric forces. Considerable opportunities for micromechanical design would be opened by pulsating Casimir plates moving back and forth entirely due to the effect of the zero-point energy, without the action of mechanical springs. This can be achieved only through the use of both attractive and repulsive Casimir forces. In connection with this, it should be noted that while the repulsive Casimir froces for a single cube or a sphere are still debated, the $\mathrm{Ca}$ simir repulsion between two parallel plates is well understood. Repulsion occurs when the plates with dielectric permittivities $\varepsilon_{1}$ and $\varepsilon_{2}$ along the imaginary frequency axis are immersed inside a medium with the dielectric permittivity $\varepsilon_{0}$ such that $\varepsilon_{1}<\varepsilon_{0}<\varepsilon_{2}$ or $\varepsilon_{2}<\varepsilon_{0}$ $<\varepsilon_{1}$ (Mahanty and Ninham, 1976). At short separations in the nonretarded van der Waals regime this effect has been long discussed and measurements have been re- 


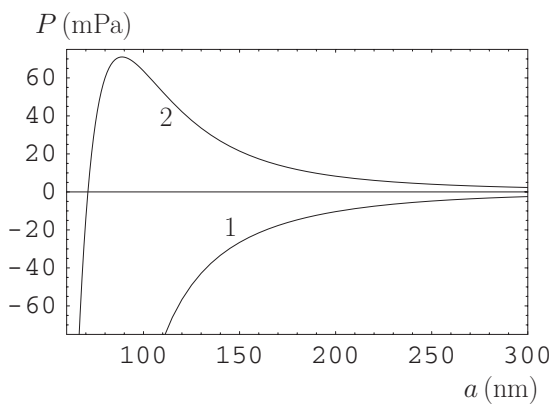

FIG. 29. The Casimir pressure vs separation in a three-layer system $\alpha-\mathrm{Al}_{5} \mathrm{O}_{2}$-ethanol-Si with no light on the Si plate (line 1) and with the illuminated Si plate (line 2).

ported [see, e.g., Visser (1981) and Meurk et al. (1997)]. At separations of about $30 \mathrm{~nm}$ the same effect was measured by Munday et al. (2009).

Recently it was shown that the illumination of one $(\mathrm{Si})$ plate in the three-layer systems Au-ethanol-Si, Siethanol-Si, and $\alpha-\mathrm{Al}_{2} \mathrm{O}_{3}$-ethanol-Si with laser pulses can change the Casimir attraction to Casimir repulsion and vice versa (Klimchitskaya et al., 2007b). The illumination can be performed as described in Sec. V.B. Calculations showed that in the system Au-ethanol-Si the force is repulsive at separations $a>160 \mathrm{~nm}$. The illumination of the Si plate, as in Sec. V.B, changes this repulsion to attraction. In the system Si-ethanol-Si the force between the Si plates is attractive, however, with one Si plate illuminated the attraction is replaced with repulsion at separations $a>175 \mathrm{~nm}$.

In the systems mentioned above, the magnitudes of the repulsive forces are several times less than the magnitude of the attractive forces at the same separations. However, it is possible to design a system where the light-induced Casimir repulsion is of the same order of magnitude as the attraction. A good example is given by the three-layer system $\alpha-\mathrm{Al}_{2} \mathrm{O}_{3}$-ethanol-Si where the $\mathrm{Si}$ plate is illuminated with laser pulses. The computational results for the Casimir pressure versus separation are presented in Fig. 29. The Casimir attraction (solid line 1) changes to repulsion at separations $a>70 \mathrm{~nm}$ when the Si plate is illuminated (solid line 2).

Note that the observation of the pulsating Casimir force requires that the plates be completely immersed in a liquid far away from any air-liquid interfaces. This prevents the occurrence of capillary forces. Surface preparation of the plates is necessary to bring about an intimate contact between the plates and the liquid. The only liquid-based force is the drag force due to the movement of the plates in response to the change of the force. For pressure values of around $10 \mathrm{mPa}$ and typical spring constants of $0.02 \mathrm{~N} / \mathrm{m}$, the corresponding drag pressure from the plate movement would be six orders of magnitude less in value. Thus, in the near future one may expect experimental confirmation of the possibility of Casimir repulsion.

\section{EXPERIMENTS ON THE CASIMIR-POLDER FORCE}

\section{A. Demonstration of the thermal Casimir-Polder force}

\section{The force in thermal equilibrium}

As mentioned in Sec. II.C, the Casimir-Polder interaction between an atom and a wall leads to a change of the center-of-mass oscillation frequency of the BoseEinstein condensate in the direction perpendicular to the wall (Antezza et al., 2004). This frequency shift can be measured precisely, leading to an indirect measurement of the Casimir-Polder force (Harber et al., 2005). Using this technique, the first measurement of the thermal Casimir-Polder force at large separations between an atom and a plate was performed by Obrecht et al. (2007). In that experiment the dipole oscillations with the frequency $\omega_{0}$ were excited in a ${ }^{87} \mathrm{Rb}$ Bose-Einstein condensate separated by a distance of a few micrometers from a fused-silica substrate (wall). The CasimirPolder force (27) between a $\mathrm{Rb}$ atom and a substrate changes the magnitude of the oscillation frequency making it equal to some $\omega_{z}$ (the $z$ direction is perpendicular to the wall). The use of the Bose-Einstein condensate is convenient because it provides a spatially compact collection of a relatively large number of atoms $\left[2.5 \times 10^{5}\right.$ atoms in the experiment by Obrecht et al. (2007)]. It is well characterized by the Thomas-Fermi density profile (29) with the radius $R_{z}=2.69 \mu \mathrm{m}$ (corresponding to a magnetic trap frequency in the radial direction $\omega_{0}=2 \pi$ $\times 229 \mathrm{~Hz}$ ). This profile is used for spatial averaging in Eq. (28).

The detailed description of the experimental setup, calibration procedures and measurements is presented by Harber et al. $(2003,2005)$ and McGuirk et al. (2004). The directly measured quantity was the relative frequency shift

$$
\gamma_{z}=\left|\omega_{0}-\omega_{z}\right| / \omega_{0}=\left|\omega_{0}^{2}-\omega_{z}^{2}\right| / 2 \omega_{0}^{2},
$$

where $\omega_{0}^{2}-\omega_{z}^{2}$ is connected [see Eq. (28)] with the spatial and time-averaged Casimir-Polder force (Antezza et al., 2004).

In thermal equilibrium the temperature of the fused silica substrate $T_{S}$ was equal to the environment temperature $T_{E}: T_{S}=T_{E}=310 \mathrm{~K}$. The frequency shift (103) was measured for a number of wall-atom (center of mass of the condensate) separations from 7 to $11 \mu \mathrm{m}$. In Fig. 30 the experimental data obtained by Obrecht et al. (2007) in thermal equilibrium at separations below $10 \mu \mathrm{m}$ are shown as crosses. Harber et al. (2005) estimated random, systematic, and total errors in the measured values of $\gamma_{z}$ in such experiments at a $66 \%$ confidence level. Obrecht et al. (2007) performed analysis for each experimental point separately. The main sources of systematic errors discussed by Harber et al. (2005) are connected with possible presence of spatially inhomogeneous electric or magnetic surface contaminations or uniform magnetic and electric fields. Special investigations were performed to obtain upper bounds on all systematic errors. In Fig. 30 the absolute total errors in the 


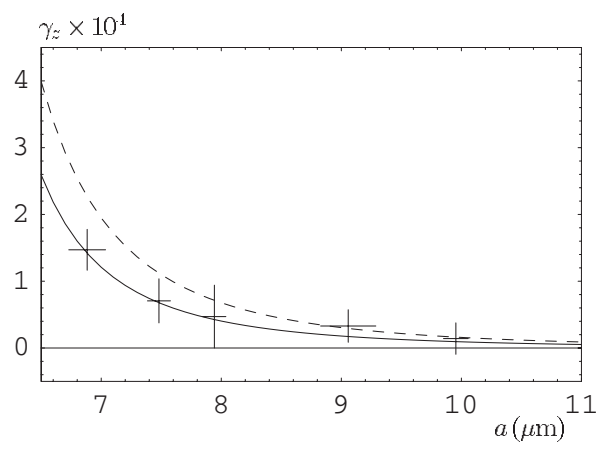

FIG. 30. The fractional change in the trap frequency vs separation in thermal equilibrium with $T_{S}=T_{E}=310 \mathrm{~K}$ computed by neglecting (solid line) and including (dashed line) the conductivity of the dielectric substrate. The experimental data are shown as crosses.

measurement of the separations and $\gamma_{z}$ are presented in true scales at each individual data point.

Comparison between the experimental data and the theory is done as in Fig. 14 (see Sec. IV.C) where the frequency shift due to the Casimir pressure between two parallel plates was shown. The measured quantity (in this case the relative frequency shift) is plotted as crosses on the same graph as the theoretical lines computed using different approaches. The theoretical values of the relative frequency shift as a function of separation are calculated using Eqs. (103) and (28) with the CasimirPolder force (27). The mass of a $\mathrm{Rb}$ atom is $m=1.443$ $\times 10^{-25} \mathrm{~kg}$. The dymanic polarizability of a $\mathrm{Rb}$ atom in Eq. (27) can be considered as frequency-independent, and the static value $\alpha\left(i \xi_{l}\right) \approx \alpha(0)=4.73 \times 10^{-23} \mathrm{~cm}^{-3}$ was used in the computations. This allows one to obtain highly accurate results for the separations under consideration (Babb et al., 2004).

Fused silica is a good insulator. However, like any insulator, it possesses a nonzero dc conductivity at nonzero temperature. Electrical conductivity in fused silica is ionic in nature and is determined by the concentration of the impurities (alkali ions) which are always present as trace constituents. At $T_{S}=T_{E}=310 \mathrm{~K}$ this conductivity varies within a wide region from $10^{-9}$ to $10^{2} \mathrm{~s}^{-1}$ (Bansal and Doremus, 1986; Shackelford and Alexander, 2001). When neglecting the dc conductivity, the dielectric permittivity of fused silica $\varepsilon\left(i \xi_{l}\right)$ as a function of $\xi_{l}$ can be calculated (Caride et al., 2005) using the tabulated optical data (Palik, 1985) and the dispersion relation (57). In this case the static dielectric permittivity has a finite value of $\varepsilon_{0}=3.81$. The respective computational results for $\gamma_{z}$ with the dc conductivity of fused silica neglected are shown in Fig. 30 by the solid line (Obrecht et al., 2007). Note that the results computed using $\varepsilon\left(i \xi_{l}\right)=\varepsilon_{0}$ (Obrecht et al., 2007) and frequency-dependent dielectric permittivity (Antezza et al., 2004; Klimchitskaya and Mostepanenko, 2008b) are almost coincident at large separation distances (only small deviations are observed at $a<8 \mu \mathrm{m})$. The theoretical computations are in good agreement with the data, as stated by Obrecht et al. (2007).
The inclusion of the dc conductivity of fused silica in the model of the dielectric response, as in Eq. (47), dramatically affects the calculations. This changes the value of the reflection coefficient $r_{\mathrm{TM}}\left(0, k_{\perp}\right)$ and, consequently, the Casimir-Polder force (27) which leads to a change in the magnitude of $\gamma_{z}$ computed using Eqs. (28) and (103). The computational results for $\gamma_{z}$ are shown in Fig. 30 as the dashed line. As shown, the first two experimental points are in disagreement with theory taking into account the conductivity of fused silica.

\section{The force out of thermal equilibrium}

Recently a nonequilibrium situation was considered where the substrate is at a temperature $T_{S}$ but the environment (remote wall) is at another temperature $T_{E}$ (Antezza et al., 2005). In this case the Casimir-Polder force has two additional terms due to the thermal fluctuations from the nearest wall at a temperature $T_{S}$ and from remote walls (environment) at a temperature $T_{E}$.

The force acting between an atom and a substrate out of thermal equilibrium depends on both temperatures and is given by (Antezza et al., 2005)

$$
F\left(a, T_{S}, T_{E}\right)=F\left(a, T_{E}\right)+F_{n}\left(a, T_{S}\right)-F_{n}\left(a, T_{E}\right) .
$$

The first term on the right-hand side of Eq. (104) is the Casimir-Polder force (27). The nonequilibrium contribution $F_{n}(a, T)$ obtained in the approximation of static atomic polarizability is defined as (Antezza et al., 2005)

$$
\begin{aligned}
& F_{n}(a, T)=-K \int_{0}^{\infty} d \omega \int_{0}^{\infty} d x f(\omega, x) e^{-2 \omega x a / c} \\
& f(\omega, x)= \frac{\omega^{4} x^{2}}{e^{\hbar \omega / k_{B} T}-1}\left[|p(\omega, x)|+\operatorname{Re} \varepsilon(\omega)-1-x^{2}\right]^{1 / 2} \\
& \times\left[\frac{1}{|\sqrt{p(\omega, x)}+i x|^{2}}\right. \\
&\left.+\frac{\left(2 x^{2}+1\right)\left[x^{2}+1+|p(\omega, x)|\right]}{|\sqrt{p(\omega, x)}+i \varepsilon(\omega) x|^{2}}\right] \\
& K \equiv \frac{2 \sqrt{2} \hbar \alpha(0)}{\pi c^{4}}, \quad p(\omega, x) \equiv \varepsilon(\omega)-1-x^{2} .
\end{aligned}
$$

The frequency shift of the condensate oscillations out of thermal equilibrium can be calculated using Eqs. (103) and (28) where $F(a, T)$ is replaced with $F\left(a, T_{S}, T_{E}\right)$ given by Eqs. (104) and (105).

In the experiment by Obrecht et al. (2007) the fusedsilica substrate was heated by the absorption with laser light. The experimental data obtained out of thermal equilibrium for $T_{E}=310 \mathrm{~K}$ and two different values of substrate temperature, $T_{S}=479$ and $605 \mathrm{~K}$, are shown as crosses in Figs. 31(a) and 31(b), respectively. As in Fig. 30 , the absolute errors are presented in true scales at each data point. The computational results for $\gamma_{z}$ in a nonequilibrium situation obtained by neglecting the dc conductivity of fused silica (Obrecht et al., 2007) are presented in Fig. 31 as the solid lines. Note that the fre- 

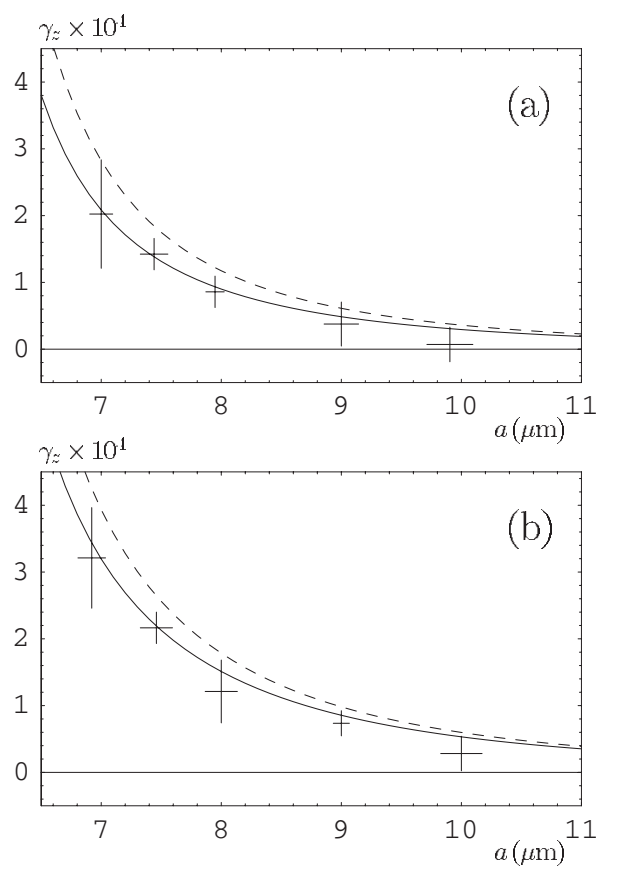

FIG. 31. The fractional change in the trap frequency vs separation out of thermal equilibrium (a) with $T_{S}=479 \mathrm{~K}$ and $T_{E}$ $=310 \mathrm{~K}$ and (b) with $T_{S}=605 \mathrm{~K}$ and $T_{E}=310 \mathrm{~K}$. Computations are done by neglecting (solid line) and including (dashed line) the conductivity of the dielectric substrate. The experimental data are shown as crosses.

quency dependence of $\varepsilon(\omega)$ in Eq. (105) does not affect the contributions to the frequency shift from the nonequilibrium terms in the total atom-wall force (104).

Direct computations show that in the nonequilibrium situation the disagreement between the experimental data and the theory with the dc conductivity of the substrate material included widens further. The corresponding results are presented in Fig. 31 as the dashed lines (Klimchitskaya and Mostepanenko, 2008b). As shown in Fig. 31(a), the three experimental points for $T_{S}=479 \mathrm{~K}$ exclude the dashed line and the other two only touch it. The dashed line in Fig. 31(b) demonstrates that all data for $T_{S}=605 \mathrm{~K}$ exclude the theoretical prediction calculated with the inclusion of the dc conductivity of fused silica. Thus, the confidence at which the theoretical approach based on Eq. (47) is excluded by data increases with the increase of substrate temperature.

It is notable that the inclusion of the dc conductivity of fused silica in the model of the dielectric response (47) does not affect the contributions to the frequency shift arising from the nonequilibrium terms $F_{n}(a, T)$ in Eq. (104) (Klimchitskaya and Mostepanenko, 2008b). Thus, the conductivity influences the computational results only through the equilibrium Casimir-Polder force (see Sec. VI.A.1). The important role of the Obrecht et al. (2007) experiment is that, not only was the model of the dielectric response taking the dc conductivity into account excluded, but the thermal effect, as predicted by the Lifshitz theory with the dc conductivity omitted, was measured for the first time.

\section{B. Future prospects to measure the Casimir-Polder force in quantum reflection}

The magnitude and the distance dependence of the Casimir-Polder force were confirmed by Sukenik et al. (1993) when studying the deflection of ground-state $\mathrm{Na}$ atoms passing through a micron-sized parallel-plate cavity. The intensity of an atomic beam transmitted through the cavity was measured as a function of the plate separation. Comparison of the experimental data with the theoretical position-dependent potential for an atom between parallel ideal mirrors (Barton, 1987a, 1987b) allowed one to investigate the atom-wall interaction at separation distances below $3 \mu \mathrm{m}$. This experiment gave impetus to the investigation of the Casimir-Polder forces in scattering experiments. Of special interest are situations when the wave nature of atom becomes dominant with respect to its classical behavior as a particle. Such a pure quantum effect is what is referred to as quantum reflection, i.e., a process in which a particle moving through a classically allowed region is reflected by a potential without reaching a classical turning point. The possibility of reflection of an ultracold atom under the influence of an attractive atom-wall interaction was predicted long ago on quantum-mechanical grounds [see, e.g., Lennard-Jones and Devonshire (1936)]. However, the experimental observation of this phenomenon has become possible only recently due to the success in the production of ultracold atoms. First it was investigated using liquid surfaces, as the reflection of $\mathrm{He}$ and $\mathrm{H}$ atoms on liquid He (Nayak et al., 1983; Berkhout et al., 1989) and from the sticking coefficient of $\mathrm{H}$ atoms on liquid He (Doyle et al., 1991; Yu et al., 1993). Later a specular reflection of very slow metastable $\mathrm{Ne}$ atoms on Si and BK7 glass surfaces was studied (Shimizu, 2001). The observed velocity dependence was explained by the quantum reflection which is caused by the attractive Casimir-Polder interaction.

Quantum reflection becomes efficient when the motion of the particle can no longer be treated semiclassically (Friedrich and Trost, 2004). The behavior of the particle is of a quantum character when

$$
\partial \lambda_{B}(z) / \partial z \geqslant 1
$$

where $\lambda_{B}(z)=2 \pi \hbar / \sqrt{2 m[E-V(z)]}$ is the local de Broglie wavelength for a particle of mass $m$ and initial kinetic energy $E$ moving in the potential $V(z)$. The same can be formulated as a condition that the variation of the local wave vector $k=2 \pi / \lambda_{B}(z)$, perpendicular to the surface, within the distance of the atomic de Broglie wavelength, is larger than $k$ itself (Shimizu, 2001),

$$
\Phi=\frac{1}{k^{2}} \frac{d k}{d z}>1 .
$$

The reflection amplitude depends critically on the energy $V(z)$ of the atom-wall interaction. This has attracted considerable attention to the theoretical investigation of the reflection amplitude depending on atomic energy and the form of the interaction potential (Friedrich et al., 2002; Jurisch and Friedrich, 2004; Voro- 
nin and Froelich, 2005; Voronin et al., 2005; Madroñero and Friedrich, 2007). The reflection probability tends to unity, as the incident velocity tends to zero. Thus, a high probability of quantum reflection calls for small incident velocities, i.e., for cold atoms.

Advances in cooling techniques in the past decade have made it possible to perform experiments with cold atoms interacting with solid surfaces. The quantum reflection of ${ }^{3} \mathrm{He}$ atoms in the scattering from an $\alpha$-quartz crystal was observed at energies far from $E \rightarrow 0$ (Druzhinina and DeKieviet, 2003). The observation of the large reflection amplitudes for the dilute BoseEinstein condensate of ${ }^{23} \mathrm{Na}$ atoms on a Si surface (Pasquini et al., 2004, 2006) allows the possibility of using quantum reflection as a trapping mechanism. The corresponding trap model has been developed theoretically (Jurisch and Friedrich, 2006; Madroñero and Friedrich, 2007). The quantum reflection of $\mathrm{He}^{*}$ atoms on a flat $\mathrm{Si}$ surface and on microfabricated surface structures was also investigated (Oberst, Kouznetsov, et al., 2005; Oberst, Taashiro, et al., 2005). The latter experiment has stimulated the development of adequate theoretical models for the description of the scattering of atomic matter waves on ridged surfaces (Kouznetsov and Oberst, 2005).

For theoretical calculations of the reflection amplitude, a simple attractive atom-wall interaction potential is commonly used (Friedrich et al., 2002; Druzhinina and DeKieviet, 2003; Oberst, Taashiro, et al., 2005) which is an interpolation between the nonretarded van der Waals energy (24) and the Casimir-Polder energy (25). Comparison of computational results with the measurement data for the reflection amplitudes allows one to estimate the parameters of the potential (Druzhinina and DeKieviet, 2003; Oberst, Taashiro, et al., 2005). The increased precision of the measurements opens new opportunities for comparison with the more exact Eq. (21) for the free energy of atom-wall interactions. Such a comparison could yield new information on the role of the atomic and material properties in dispersion forces. As shown by Bezerra et al. (2008), for an atom of metastable $\mathrm{He}^{*}$ and a $\mathrm{Au}$ wall at zero temperature, the largest deviations between the phenomenological potential and the exact interaction energy of about $10 \%$ are achieved at separations of $300-500 \mathrm{~nm}$. However, at room temperature the free energy deviates from the phenomenological potential by $31 \%$ at $a=5 \mu \mathrm{m}$. There is also related theoretical research on Casimir physics in atom-wall interaction devoted to the influence of nonzero temperature on atomic polarizability (Buhmann and Scheel, 2008), the use of the lateral Casimir-Polder force between an atom, and a corrugated surface to study nontrivial geometrical effects (Dalvit et al., 2008) and to the interaction of atoms with conducting microstructures (Eberlein and Zietal, 2007).

\section{Casimir-Polder interaction of atoms with carbon nanotubes}

Experiments with ultracold atoms have generated interest on the scattering and trapping of such atoms by different nanostructures (Arnecke et al., 2007; Fermani et al., 2007). In this case, the probability of quantum reflection is sensitive to the shape of the potential (Friedrich et al., 2002; Arnecke et al., 2007). Thus, it needs to be ascertained if the general theory of dispersion forces can describe the interaction between microparticles and nanostructures. An additional question to be answered is to what extent the macroscopic concept of the dielectric permittivity can be used as an adequate characteristic of nanostructure material properties.

As a first step in this direction, the Lifshitz theory of the van der Waals force has been extended for the case of a microparticle interacting with a plane surface of a uniaxial crystal or with a cylindrical shell made of such crystal (Blagov et al., 2005). An approximate expression for the free energy of a microparticle-cylinder interaction was obtained using the PFA which is of high precision at microparticle-cylinder separations smaller than the cylinder radius. The reflection coefficients from the surface of a uniaxial crystal (with the optical axis $z$ perpendicular to the surface) can be expressed in terms of two dissimilar dielectric permittivities $\varepsilon_{x}(\omega)$ and $\varepsilon_{z}(\omega)$. For example, the multilayer graphite cylinder was considered as a simple model of a multiwalled carbon nanotube. Using this model, the Casimir-Polder interaction between hydrogen atoms (molecules) and multiwalled carbon nanotubes was investigated (Blagov et al., 2005; Klimchitskaya, Blagov, and Mostepanenko, 2006). Later, Lifshitz-type formulas have been obtained which describe the van der Waals and Casimir-Polder interaction between a graphene sheet and a material plate or a microparticle (Bordag et al., 2006) and a microparticle and a single-walled carbon nanotube (Blagov et al., 2007). For this purpose graphene was considered as an infinitesimally thin positive charged flat sheet, carrying a continuous fluid with some mass and negative charge densities. This plasma sheet is characterized by a typical wave number $K$ determined by the parameters of the hexagonal structure of graphite. The interaction of the electromagnetic oscillations with such a sheet was considered and the normal modes and reflection coefficients were found (Barton, 2004, 2005).

A single-walled carbon nanotube can be approximately modeled as a cylindrical sheet carrying a twodimensional free-electron gas with the same reflection coefficients as for a plane sheet (Blagov et al., 2007). Note that the above approximate models do not take into account nanotube chirality (Saito et al., 1998). This can be included using the optical data for the nanotube index of refraction. Regarding single-walled carbon nanotubes, it remains unclear if it is possible to describe nanotubes with surfaces such as metals or semiconductors by varying only one parameter, the typical wave number $K$, in the reflection coefficients.

By comparing the calculational results for the multiwalled nanotubes with those for single-walled, it was demonstrated that the macroscopic desciption using the concept of dielectric permittivity is applicable for nanotubes with only two or three layers at separation distances larger than $2 \mathrm{~nm}$ (Blagov et al., 2007; Klim- 
chitskaya, Blagov, and Mostepanenko, 2008). It is well to bear in mind that the description of multiwalled nanotubes by the graphite dielectric permittivity and singlewalled nanotubes as a two-dimensional free electron gas are only models and are not a complete description of all the nanotube properties. Nevertheless, they are good approximations for the estimation of microparticlenanotube interaction on the basis of the Lifshitz theory.

\section{LATERAL CASIMIR FORCE AND CASIMIR TORQUES}

\section{A. Lateral Casimir force between corrugated surfaces}

The Casimir force considered in the preceding sections is the normal one, i.e., it leads to an attraction perpendicular to the surfaces of the interacting bodies. However, as mentioned in Sec. I.B, there is a geometry dependence for the Casimir force on the shape of the surface, especially for corrugated surfaces. Thus, the nontrivial boundary dependence of the normal Casimir force acting between a plate with periodic uniaxial sinusoidal corrugations and a large sphere was demonstrated by Roy and Mohideen (1999). It follows also from Eq. (67) that the pressure between two corrugated plates with parallel uniaxial sinusoidal corrugations of the same period harmonically depends on the phase shift between the corrugations (Bordag et al., 1995a). This should result in the lateral Casimir force in addition to the normal one.

Using the path integral method, Golestanian and Kardar $(1997,1998)$ predicted the existence of the lateral Casimir force in the configuration of two corrugated plates. Later the geometry dependence of the Casimir energy for sinusoidally corrugated ideal metal plates was studied by the path integral quantization of the electromagnetic field (Emig et al., 2001, 2003). The general expressions for both the normal and the lateral Casimir force were obtained in the lowest order of perturbation theory with respect to the relative corrugation amplitudes $A_{1}$ and $A_{2}$. Here the interacting surfaces were described by Eq. (63) with $f_{1}(x, y)=\sin (2 \pi x / \Lambda), f_{2}(x, y)$ $=\sin \left[2 \pi\left(x+x_{0}\right) / \Lambda\right]$. A harmonic dependence of the lateral force on the phase shift $\varphi=2 \pi x_{0} / \Lambda$ was obtained (Emig et al., 2001, 2003). Later, the exact solution was found for the lateral Casimir force in the configuration of two parallel ideal metal plates with laterally shifted uniaxial rectangular gratings (Büscher and Emig, 2005).

The influence of the lateral force acting on a sphere above a corrugated plate can explain (Klimchitskaya et al., 2001) the nontrivial character of the normal Casimir force in this configuration experimentally demonstrated by Roy and Mohideen (1999).

All theoretical results on the lateral Casimir force mentioned above were obtained for uniaxial corrugations of equal period. If the periods of corrugations are different, the lateral force becomes equal to zero. The uniaxial character of the corrugations is also important for the observation of the lateral Casimir force. Under the assumption that there is a nonzero angle $\vartheta$ between

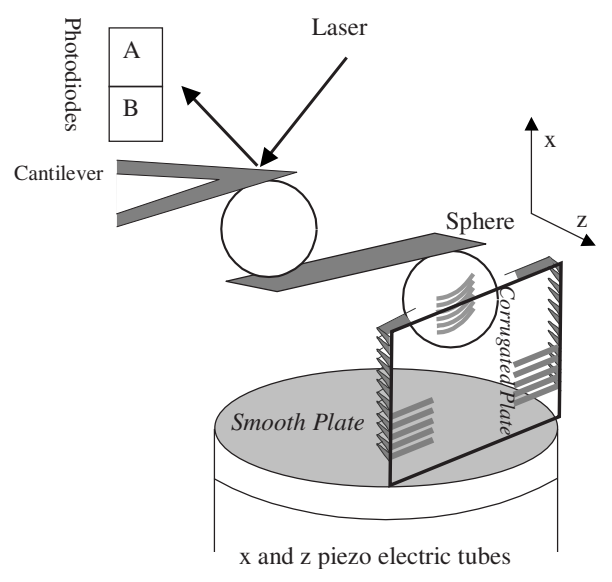

FIG. 32. Schematic of the experimental setup for measuring the lateral Casimir force. The $x$ piezo and $z$ piezo are independent.

the axes of the corrugations, the phase shift along the $x$ axis becomes the periodic function of $y$ with a period $\Lambda_{y}=\Lambda \cot \vartheta$. Within one period $\varphi(y)$ depends on $y$ linearly, taking on values from 0 to $2 \pi$. The resulting lateral force should be averaged over the period $\Lambda_{y}$. In the case of an infinite plate this leads to a zero value at any $\vartheta \neq 0$. For real bodies of finite size the lateral Casimir force will be measurable only for small deviations of the corrugation axes from parallelity such that $\Lambda \cot \vartheta$ is much larger than the size of the smallest body. For example, for $\Lambda \approx 1 \mu \mathrm{m}$ and a body size of $10 \mu \mathrm{m}$ the lateral Casimir force is observable only if $\vartheta \ll 0.1 \mathrm{rad}$.

The existence of the lateral Casimir force opens new opportunities for the application of the Casimir force in micromachines (Ashourvan et al., 2007a, 2007b; Emig, 2007). It is also important that this force can be well controlled by specific shapes of corrugations (Blagov et $a l ., 2004)$. Based on the path integral theory (Emig et al., 2003) it appears that the lateral Casimir force is a promising venue for the demonstration of shape dependences and diffractionlike effects. For these reasons an experimental investigation of the lateral Casimir force is of much interest.

\section{B. Demonstration of the lateral Casimir force}

The lateral Casimir force between a $\mathrm{Au}$ plate and a sphere, both sinusoidally corrugated, was measured for surface separations between 200 and $300 \mathrm{~nm}$ using an AFM (Chen et al., 2002a, 2002b). The experiment was performed at a pressure below 50 mtorr and at room temperature. A schematic diagram of the experiment is shown in Fig. 32. To implement this experiment a diffraction grating with uniaxial sinusoidal corrugations of period $\Lambda=1.2 \mu \mathrm{m}$ and an amplitude of $90 \mathrm{~nm}$ was used as the template. In order to obtain perfect orientation and phase between the corrugated surfaces, a special imprinting procedure was developed (Chen et al., 2002a, 2002b). The amplitude of the corrugations on the plate and that imprinted on the sphere were measured using 


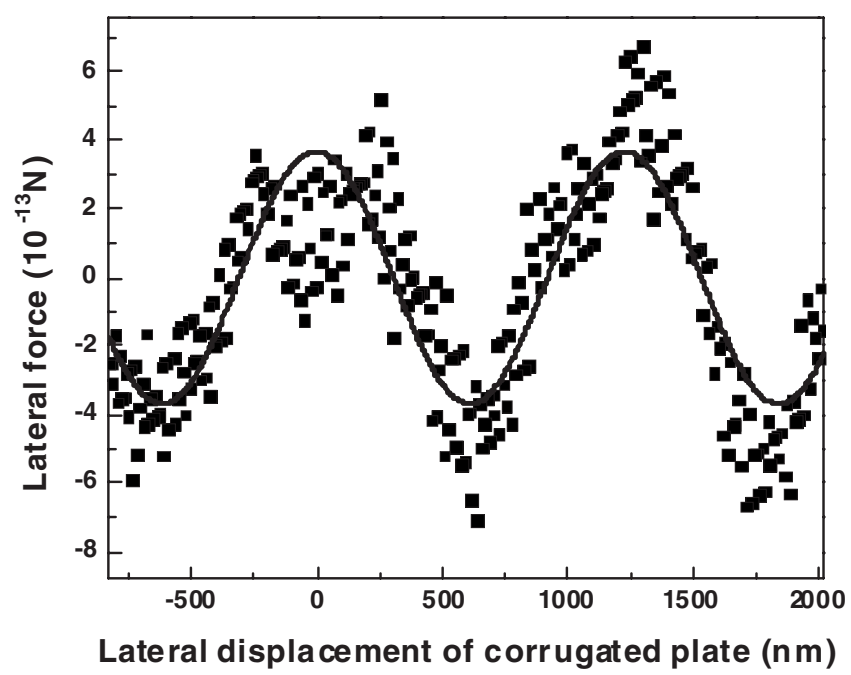

FIG. 33. Comparison of experiment and theory. The average measured lateral Casimir force as a function of the lateral displacement is shown as solid squares. The solid line is the best fit sine curve to the data leading to a lateral force amplitude of $0.32 \mathrm{pN}$.

the AFM to be $59 \pm 7$ and $8 \pm 1 \mathrm{~nm}$, respectively. The calibration of the cantilever and the measurement of the residual potential voltage between the sphere and the plate were done by electrostatic means in a manner similar to experiments on measuring the normal Casimir force (see Sec. IV.A).

The corrugated plate was mounted on two piezos that allowed independent movement of the plate in the vertical and horizontal directions with the help of the $x$ piezo and $z$ piezo, respectively. Movement in the $x$ direction is necessary to achieve a lateral phase shift between the corrugations on the sphere and the plate. Independent movement in the $z$ direction is necessary for control of the surface separation. The lateral Casimir force was measured at four different separations starting from $221 \mathrm{~nm}$ with steps of $12 \mathrm{~nm}$. At each separation the measurement was repeated 60 times and the averaged force was determined. The averaged lateral force measured at a separation $221 \mathrm{~nm}$ is shown as solid squares in Fig. 33. The sinusoidal oscillations as a function of the phase difference between the two corrugations are clearly observed. The periodicity of the lateral force oscillations is in agreement with the corrugation period of the plate. A sine curve fit to the observed data is shown in Fig. 33 as the solid line and corresponds to an amplitude of $0.32 \mathrm{pN}$. Detailed error analysis (Chen et al., $2002 \mathrm{~b}$ ) led to the total absolute experimental error of $0.077 \mathrm{pN}$ at a $95 \%$ confidence level. The resulting precision of the amplitude measurements at the closest separation is around $24 \%$.

The measurement data were compared with theoretical results. Note that the measurements were performed at separations from 200 to $300 \mathrm{~nm}$ where the corrections due to the skin depth are rather large (see Sec. IV.A). In addition, the ratio of the corrugation amplitude on the plate to the separation distance is not a small parameter
( $\left.A_{1} / a \approx 0.27\right)$. For this reason, it is not appropriate to compare experimental results with theoretical predictions obtained for ideal metal test bodies in the lowestorder perturbation theory. However, separations are several times smaller than the corrugation period $\Lambda$. Because of this, diffractionlike effects do not contribute (see Sec. III.B). In this case the theoretical expression for the lateral Casimir force including corrections to the skin depth can be obtained in the form

$$
F^{\text {lat }}(a, \varphi)=\frac{\pi^{4} R \hbar c}{120 a^{4} \Lambda} A_{1} A_{2} G\left(a, \Lambda, A_{1}, A_{2}\right) \sin \varphi .
$$

The explicit form of the function $G$ is different based on whether the proximity force approximation or the pairwise summation method is used. In the framework of the PFA $G$ does not depend on $\Lambda$ (Chen et al., 2002a, $2002 b)$. The resulting theoretical values for the amplitude of the lateral Casimir force at $a=221 \mathrm{~nm}$ are 0.27 and $0.31 \mathrm{pN}$ when calculated up to the second and fourth perturbation orders in the small parameters $A_{i} / a$, respectively. Thus, the fourth-order term is $15 \%$ of the second-order one and cannot be neglected.

The pairwise summation method is applicable to larger ratios of $a / \Lambda$ and the results obtained depend on $\Lambda$. Using this method, the explicit expression for the function $G$ in Eq. (108) is

$$
\begin{aligned}
G= & \kappa_{E}^{(s p)}(a)\left[S_{\mathrm{pw}}^{(1)}\left(2 \pi \frac{a}{\Lambda}\right)+\frac{5}{2} S_{\mathrm{pw}}^{(2)}\left(2 \pi \frac{a}{\Lambda}\right) \frac{A_{1}^{2}+A_{2}^{2}}{a^{2}}\right. \\
& \left.-5 \cos \delta S_{\mathrm{pw}}^{(2)}\left(4 \pi \frac{a}{\Lambda}\right) \frac{A_{1} A_{2}}{a^{2}}\right] .
\end{aligned}
$$

Here the coefficients depending on $a / \Lambda$ are given by

$$
\begin{aligned}
S_{\mathrm{pw}}^{(1)}(x)= & e^{-x}\left(1+x+\frac{x^{2}}{6}-\frac{x^{3}}{6}\right)+\frac{x^{4}}{6} \Gamma(0, x) \\
S_{\mathrm{pw}}^{(2)}(x)= & e^{-x}\left(1+x+\frac{31}{80} x^{2}+\frac{13}{240} x^{3}-\frac{x^{4}}{480}+\frac{x^{5}}{480}\right) \\
& -\frac{x^{6}}{480} \Gamma(0, x)
\end{aligned}
$$

and $\Gamma(\alpha, x)$ is the incomplete Gamma function. The correction factor $\kappa_{E}^{(s p)}$ to the Casimir energy due to the nonzero skin depth in the configuration of a sphere above a plate is defined by

$$
-\int_{a}^{\infty} E\left(a^{\prime}\right) d a^{\prime}=\frac{\pi^{2} \hbar c}{1440 a^{2}} \kappa_{E}^{(s p)}(a),
$$

where $E(a)$ is the Casimir energy per unit area of two parallel plates presented in Eq. (12). Using Eqs. (108) and (109) at $a=221 \mathrm{~nm}$, one obtains the amplitude of the lateral Casimir force equal to 0.24 and $0.30 \mathrm{pN}$ in the second and fourth perturbation orders in $A_{i} / a$, respectively. Here the fourth-order term is $25 \%$ of the secondorder result. It is notable that the amplitudes of the lateral Casimir force computed using both approximate methods up to the fourth perturbation order in $A_{i} / a$ are 
almost equal and in good agreement with the measurement result. The force amplitudes measured at different separations demonstrated the $a^{-4}$ dependence in agreement with theoretical predictions.

Recently a theoretical approach was developed (Rodrigues et al., 2006a; Rodrigues, Maia Neto, et al., 2007) which allows one to calculate the lateral Casimir force between sinusoidally corrugated plates made of metals described by the plasma model [Eq. (32)] without using the PFA or pairwise summation but only in the lowest second perturbation order under the conditions $A_{1}, A_{2}$ $\ll \lambda_{p}, a, \Lambda$. These conditions are not satisfied in the experiment discussed above (specifically in that experiment $\left.A_{1} / a \approx 0.27, A_{1} / \lambda_{p} \approx 0.43\right)$. As we have seen, the contributions of the fourth perturbation orders in $A_{i} / a$ are important. Because of this, comparison of the experimental data with the theoretical predictions (Rodrigues et al., 2006a; Rodrigues, Maia Neto, et al., 2007) is not appropriate (Chen et al., 2007).

\section{The Casimir torque}

Another interesting effect that predicted long ago but has not yet been observed is the Casimir torque. Such a torque arises due to the zero-point electromagnetic oscillations in the configuration of anisotropic or asymmetric bodies. In the case of plates made of a uniaxial crystal described by the dielectric permittivities $\varepsilon_{x}(\omega)$ and $\varepsilon_{y}(\omega)=\varepsilon_{z}(\omega)$, the torque arises if there is a nonzero angle $\vartheta$ between the optical axes of the plates. In the nonretarded limit the Casimir torque was investigated by Parsegian and Weiss (1972). The retardation effects were taken into account by Barash (1973). Recently these results were obtained using another method (Philbin and Leonhardt, 2008). The torque leads to the rotation of the plates until their optical axes are aligned. In the nonretarded limit and for small anisotropies $\left|\varepsilon_{x}-\varepsilon_{y}\right| / \varepsilon_{y} \ll 1$ the torque between the test bodies of area $S$ at a separation $a$ is $M \sim-S \sin (2 \vartheta) / a^{2}$ (Munday et al., 2005). In the relativistic limit $M \sim-S \sin (2 \vartheta) / a^{3}$ (Mostepanenko and Trunov, 1997).

Recently an interesting experimental scheme was proposed to observe this torque when a barium titanate plate is immersed in ethanol and an anisotropic disk is placed above (Munday et al., 2005). The dielectric permittivities are chosen in such a way that the Casimir force between the anisotropic bodies is repulsive (see Sec. V.E.3). The disk would float parallel to the plate at a distance where its weight is counterbalanced by the Casimir repulsion, being free to rotate in response to a small torque. Detailed numerical calculations are made demonstrating the feasibility of this experiment (Munday et al., 2005).

Another possibility to observe the Casimir torque was theoretically investigated in the configuration of two corrugated plates with a small angle between the corrugation directions (Rodrigues et al., 2006b). As discussed in Sec. 7.A, the lateral Casimir force and related torque differ from zero for an angle between corrugation axes $\vartheta \ll \Lambda / L$, where $L$ is the size of the plate along the corrugation axis. The optimum values of the corrugation period $\Lambda$ and plate separations were found in order to get larger values of the Casimir torque in comparison with those predicted for anisotropic test bodies of the same area (Rodrigues et al., 2006b).

Both experiments, if successfully performed, will provide important new information about dispersion forces between real materials. From an application standpoint, the Casimir torque may serve as one more mechanism of control in micromachines.

\section{CONCLUSIONS AND OUTLOOK}

In the foregoing, the intersection of experiment and theory in the investigation of the Casimir force between real material bodies has been reviewed. In the last ten years the field has undergone many rapid advances. Many new results concerning the Casimir effect of both a fundamental and applied character have been obtained and were presented above. They are related to condensed matter physics, statistical physics, atomic physics, and nanotechnology. This list should be supplemented with elementary particle physics, quantum field theory, gravitation and cosmology, and mathematical physics if one keeps in mind that many important results on the Casimir effect are not directly connected with experiments using real materials and, consequently, are not covered in this review.

Although experimental Casimir physics is still a young field, an important period in its development has been completed and the related conclusions can be formulated. The most important of these conclusions is that the Casimir force between real materials has been experimentally measured by many using different techniques, and the data obtained demonstrate the role of real material properties and the geometrical shape of the test bodies (see Secs. IV.A-IV.C, V.B-V.D, and VII.B). Although there are still different opinions on the measure of agreement between experiment and theory that has been achieved, statistical procedures for data processing and for comparison between the experimental and theoretical results in precise force-distance measurements have been developed (see Sec. III.C) and successfully used in several experiments (see Secs. IV.A, IV.B, V.B, and V.C). Another important conclusion is that the application of the general Lifshitz theory of dispersion forces to real materials at nonzero temperature results in puzzles. These puzzles are of both theoretical and experimental character. On the theoretical side, an enormously large thermal effect was predicted, arising when physically real but seemingly negligible properties of real dielectrics are taken into account. A related thermal effect also arises for Drude metals, and both lead to a thermodynamic inconsistency (Sec. II.D). Experimentally, the predicted large thermal effects were excluded by measurements of different groups performed with metal, dielectric, and semiconductor test bodies (see Secs. IV.B, VI.A, and V.B, respectively). If some specific properties of the real bodies (the dc conductivity of di- 
electrics, which vanishes with vanishing temperature, or relaxation properties of conduction electrons in metals) are disregarded, the Lifshitz theory is found to be in good agreement with all performed experiments and with thermodynamics. As argued in Sec. II.D.1, the dielectric permittivities used to describe the drift and diffusion currents in dielectrics and metals are not compatible with the Lifshitz theory because the existence of these currents violates the state of thermal equilibrium. This suggests that one should not include the drift and diffusion currents in the model of the dielectric response when applying the Lifshitz theory to real materials. There is no current consensus with respect to this inference.

One can conclude that the current Lifshitz theory, if applied in accordance with its basic postulates, is consistent with the principles of thermodynamics and all available experimental data. Some claims may be raised because this theory does not include the conductivity properties of real materials. However, the experimental data testify that these properties have nothing to do with dispersion forces. We believe that a future theory of dispersion forces will not use reflection coefficients expressed in terms of dielectric permittivity but will deal with more general characteristics of scattering processes allowing, in particular, the inclusion of spatial nonlocality.

Many other future applications of dispersion forces in real systems have been discussed but have not yet been investigated experimentally. Because of this, they are not reflected in our review. One might mention the van der Waals and Casimir interatomic interaction in a magnetodielectric medium (Spagnolo et al., 2007), the Casimir-Polder forces of excited atoms (Buhmann and Welsch, 2008), and the Casimir interaction between plates with dielectric permittivity and magnetic permeability (Kenneth et al., 2002; Pirozhenko and Lambrecht, 2008b). Artificially microstructured metamaterials (Pendry et al., 1999) are also promising for use in studies of the Casimir effect. For example, the theoretical possibility that metamaterials might lead to repulsive Casimir forces in some distance ranges has been investigated (Henkel and Joulain, 2005; Leonhardt and Philbin, 2007; Rosa et al., 2008). It was pointed out that a strong modification of the Casimir force is possible for surface separations around the resonance wavelength of the magnetic response. Note that natural materials are physically limited to magnetic permeabilities $\mu$ of order of unity in the visible spectrum. However, artificially designed micrometer and submicrometer split-ring resonators and fish-net arrays with $\mu>1$ have been reported with resonances in the infrared and near-optical frequencies (Shalaev, 2007). Rosa et al. (2008) simulated the Casimir force between a metamaterial slab and a half space of Ag. A repulsive Casimir force was found for a short band of separations only if there is no background metallic response component. It was pointed out that the large dissipation that is present in metamaterials (Chettiar et al., 2007) would substantially reduce any Casimir repulsion. It should also be noted that presently fabricated metamaterials in the visible frequency range have a very narrow frequency region where $\mu>1$ (Chettiar et al., 2007). Thus many engineering challenges remain for design of appropriate materials. Nevertheless, they show great potential for exploring new Casimir effect phenomena. Future prospects for measurement of the thermal Casimir force, the Casimir force between semiconductor surfaces and the Casimir-Polder force in quantum reflection have already been discussed in Secs. IV.D, V.E, and VI.B, respectively. This means we look forward to many exciting applications of the Casimir force in the near future.

\section{ACKNOWLEDGMENTS}

We are grateful to many colleagues for numerious helpful discussions. We express special gratitude to J. F. Babb, V. B. Bezerra, G. Bimonte, E. V. Blagov, M. Bordag, F. Chen, R. S. Decca, E. Fischbach, B. Geyer, D. E. Krause, D. López, and C. Romero. We are also grateful to M. Antezza, G. Carugno, G. Jourdan, and R. Wild for important additional information about their experiments. This work was supported by the National Science Foundation (Grant No. PHY0653657), Department of Energy (Grant No. DE-FG02-04ER46131), and Deutsche Forschungsgemeinschaft (Grant No. 436 RUS 113/789/0-4).

\section{REFERENCES}

Agranovich, V. M., and V. L. Ginzburg, 1984, Crystal Optics with Spatial Dispersion (Springer, Berlin).

Antezza, M., L. P. Pitaevskii, and S. Stringari, 2004, Phys. Rev. A 70, 053619.

Antezza, M., L. P. Pitaevskii, and S. Stringari, 2005, Phys. Rev. Lett. 95, 113202.

Antonini, P., G. Bressi, G. Carugno, G. Galeazzi, G. Messineo, and G. Ruoso, 2006, New J. Phys. 8, 239.

Arnecke, F., H. Friedrich, and J. Madroñero, 2007, Phys. Rev. A 75, 042903.

Arnold, W., S. Hunklinger, and K. Dransfeld, 1979, Phys. Rev. B 19, 6049.

Ashcroft, N. W., and N. D. Mermin, 1976, Solid State Physics (Saunders College, Philadelphia).

Ashourvan, A., M. Miri, and R. Golestanian, 2007a, Phys. Rev. Lett. 98, 140801.

Ashourvan, A., M. Miri, and R. Golestanian, 2007b, Phys. Rev. E 75, 040103.

Babb, J. F., G. L. Klimchitskaya, and V. M. Mostepanenko, 2004, Phys. Rev. A 70, 042901.

Balian, R., and B. Duplantier, 1977, Ann. Phys. (N.Y.) 104, 300. Balian, R., and B. Duplantier, 1978, Ann. Phys. (N.Y.) 112, 165.

Bansal, N. P., and R. H. Doremus, 1986, Handbook of Glass Properties (Academic, New York).

Barash, Yu. S., 1973, Izv. Vyssh. Uchebn. Zaved., Radiofiz. 16, 1227 [ Sov. Radiophys. 16, 945 (1973)].

Barash, Yu. S., and V. L. Ginzburg, 1975, Usp. Fiz. Nauk 116, 5 [Sov. Phys. Usp. 18, 305 (1975)].

Barton, G., 1987a, Proc. R. Soc. London, Ser. A 410, 141. Barton, G., 1987b, Proc. R. Soc. London, Ser. A 410, 175. Barton, G., 2004, J. Phys. A 37, 1011. 
Barton, G., 2005, J. Phys. A 38, 2997.

Berger, J., 1993, Statistical Decision Theory and Bayesian Analysis (Springer, New York).

Bergström, L., 1997, Adv. Colloid Interface Sci. 70, 125.

Berkhout, J. J., O. J. Luiten, I. D. Setija, T. W. Hijmans, T. Mizusaki, and J. T. M. Walraven, 1989, Phys. Rev. Lett. 63, 1689.

Bezerra, V. B., G. Bimonte, G. L. Klimchitskaya, V. M. Mostepanenko, and C. Romero, 2007, Eur. Phys. J. C 52, 701.

Bezerra, V. B., R. S. Decca, E. Fischbach, B. Geyer, G. L. Klimchitskaya, D. E. Krause, D. López, V. M. Mostepanenko, and C. Romero, 2006, Phys. Rev. E 73, 028101.

Bezerra, V. B., G. L. Klimchitskaya, and V. M. Mostepanenko, 2000, Phys. Rev. A 62, 014102.

Bezerra, V. B., G. L. Klimchitskaya, and V. M. Mostepanenko, 2002a, Phys. Rev. A 65, 052113.

Bezerra, V. B., G. L. Klimchitskaya, and V. M. Mostepanenko, 2002b, Phys. Rev. A 66, 062112.

Bezerra, V. B., G. L. Klimchitskaya, V. M. Mostepanenko, and C. Romero, 2004, Phys. Rev. A 69, 022119.

Bezerra, V. B., G. L. Klimchitskaya, V. M. Mostepanenko, and C. Romero, 2008, Phys. Rev. A 78, 042901.

Bezerra, V. B., G. L. Klimchitskaya, and C. Romero, 1997, Mod. Phys. Lett. A 12, 2613.

Bezerra, V. B., G. L. Klimchitskaya, and C. Romero, 2000, Phys. Rev. A 61, 022115.

Bezerra, V. B., G. L. Klimchitskaya, and C. Romero, 2001, Phys. Rev. A 65, 012111.

Bimonte, G., 2006a, Phys. Rev. Lett. 96, 160401.

Bimonte, G., 2006b, Phys. Rev. E 73, 048101.

Bimonte, G., 2007, New J. Phys. 9, 281.

Bimonte, G., 2008, Phys. Rev. A 78, 062101.

Bimonte, G., E. Calloni, G. Esposito, L. Milano, and L. Rosa, 2005, Phys. Rev. Lett. 94, 180402.

Bimonte, G., E. Calloni, G. Esposito, and L. Rosa, 2005, Nucl. Phys. B 726, 441.

Bingqian, L., Z. Changchun, and L. Junhua, 1999, J. Micromech. Microeng. 9, 319.

Blagov, E. V., G. L. Klimchitskaya, U. Mohideen, and V. M. Mostepanenko, 2004, Phys. Rev. A 69, 044103.

Blagov, E. V., G. L. Klimchitskaya, and V. M. Mostepanenko, 2005, Phys. Rev. B 71, 235401.

Blagov, E. V., G. L. Klimchitskaya, and V. M. Mostepanenko, 2007, Phys. Rev. B 75, 235413.

Blocki, J., J. Randrup, W. J. Swiatecki, and C. F. Tsang, 1977, Ann. Phys. (N.Y.) 105, 427.

Bohren, C. F., and D. R. Huffmann, 1998, Absorption and Scattering of Light by Small Particles (Wiley, New York).

Bordag, M., 2006, Phys. Rev. D 73, 125018.

Bordag, M., B. Geyer, G. L. Klimchitskaya, and V. M. Mostepanenko, 1998, Phys. Rev. D 58, 075003.

Bordag, M., B. Geyer, G. L. Klimchitskaya, and V. M. Mostepanenko, 1999, Phys. Rev. D 60, 055004.

Bordag, M., B. Geyer, G. L. Klimchitskaya, and V. M. Mostepanenko, 2000a, Phys. Rev. D 62, 011701(R).

Bordag, M., B. Geyer, G. L. Klimchitskaya, and V. M. Mostepanenko, 2000b, Phys. Rev. Lett. 85, 503.

Bordag, M., B. Geyer, G. L. Klimchitskaya, and V. M. Mostepanenko, 2006, Phys. Rev. B 74, 205431.

Bordag, M., G. L. Klimchitskaya, and V. M. Mostepanenko, 1994, Mod. Phys. Lett. A 9, 2515.

Bordag, M., G. L. Klimchitskaya, and V. M. Mostepanenko, 1995a, Int. J. Mod. Phys. A 10, 2661.
Bordag, M., G. L. Klimchitskaya, and V. M. Mostepanenko, 1995b, Phys. Lett. A 200, 95.

Bordag, M., U. Mohideen, and V. M. Mostepanenko, 2001, Phys. Rep. 353, 1.

Boström, M., and B. E. Sernelius, 2000, Phys. Rev. Lett. 84, 4757.

Boström, M., and B. E. Sernelius, 2004, Physica A 339, 53.

Boyer, T. H., 1968, Phys. Rev. 174, 1764.

Brandt, S., 1976, Statistical and Computational Methods in Data Analysis (North-Holland, Amsterdam).

Bressi, G., G. Carugno, R. Onofrio, and G. Ruoso, 2002, Phys. Rev. Lett. 88, 041804.

Brevik, I., J. B. Aarseth, J. S. Høye, and K. A. Milton, 2005, Phys. Rev. E 71, 056101.

Brevik, I., S. A. Ellingsen, J. S. Høye, and K. A. Milton, 2008, J. Phys. A: Math. Theor. 41, 164017.

Brown, L. S., and G. J. Maclay, 1969, Phys. Rev. 184, 1272.

Brown-Hayes, M., D. A. R. Dalvit, F. D. Mazzitelli, W. J. Kim, and R. Onofrio, 2005, Phys. Rev. A 72, 052102.

Brownlee, K. A., 1965, Statistical Theory and Methodology in Science and Engineering (Wiley, New York).

Bryksin, V. V., and P. M. Petrov, 2008, Fiz. Tverd. Tela (St. Petersburg) 50, 222 [Phys. Solid State 50, 229 (2008)].

Buhmann, S. Y., and S. Scheel, 2008, Phys. Rev. Lett. 100, 253201.

Buhmann, S. Y., and D.-G. Welsch, 2007, Prog. Quantum Electron. 31, 51.

Buhmann, S. Y., and D.-G. Welsch, 2008, Phys. Rev. A 77, 012110.

Buhmann, S. Y., D.-G. Welsch, and T. Kampf, 2005, Phys. Rev. A 72, 032112.

Buks, E., and M. L. Roukes, 2001, Phys. Rev. B 63, 033402.

Buks, E., and M. L. Roukes, 2002, Nature (London) 419, 119.

Bulgac, A., P. Magierski, and A. Wirzba, 2006, Phys. Rev. D 73, 025007.

Büscher, R., and T. Emig, 2004, Phys. Rev. A 69, 062101.

Büscher, R., and T. Emig, 2005, Phys. Rev. Lett. 94, 133901.

Capasso, F., J. N. Munday, D. Iannuzzi, and H. B. Chan, 2007, IEEE J. Quantum Electron. 13, 400.

Caride, A. O., G. L. Klimchitskaya, V. M. Mostepanenko, and S. I. Zanette, 2005, Phys. Rev. A 71, 042901.

Carlin, B. P., and T. A. Louis, 2000, Bayes and Empirical Bayes Methods of Data Analysis (CRC, Boca Raton).

Casimir, H. B. G., 1948, Proc. K. Ned. Akad. Wet. Ser. B: Phys. Sci. 51, 793.

Casimir, H. B. G., and D. Polder, 1948, Phys. Rev. 73, 360.

Castillo-Garza, R., C.-C. Chang, D. Jimenez, G. L. Klimchitskaya, V. M. Mostepanenko, and U. Mohideen, 2007, Phys. Rev. A 75, 062114.

Chan, H. B., V. A. Aksyuk, R. N. Kleiman, D. J. Bishop, and F. Capasso, 2001a, Science 291, 1941.

Chan, H. B., V. A. Aksyuk, R. N. Kleiman, D. J. Bishop, and F. Capasso, 2001b, Phys. Rev. Lett. 87, 211801.

Chan, H. B., Y. Bao, J. Zou, R. A. Cirelli, F. Clemens, W. M. Mansfield, and C. S. Pai, 2008, Phys. Rev. Lett. 101, 030401. Chazalviel, J.-N., 1999, Coulomb Screening of Mobile Charges: Applications to Material Science, Chemistry and Biology (Birkhauser, Boston).

Chen, F., G. L. Klimchitskaya, U. Mohideen, and V. M. Mostepanenko, 2003, Phys. Rev. Lett. 90, 160404.

Chen, F., G. L. Klimchitskaya, U. Mohideen, and V. M. Mostepanenko, 2004, Phys. Rev. A 69, 022117.

Chen, F., G. L. Klimchitskaya, V. M. Mostepanenko, and U. 
Mohideen, 2006, Phys. Rev. Lett. 97, 170402.

Chen, F., G. L. Klimchitskaya, V. M. Mostepanenko, and U. Mohideen, 2007a, Opt. Express 15, 4823.

Chen, F., G. L. Klimchitskaya, V. M. Mostepanenko, and U. Mohideen, 2007b, Phys. Rev. B 76, 035338.

Chen, F., and U. Mohideen, 2001, Rev. Sci. Instrum. 72, 3100.

Chen, F., U. Mohideen, G. L. Klimchitskaya, and V. M. Mostepanenko, 2002a, Phys. Rev. Lett. 88, 101801.

Chen, F., U. Mohideen, G. L. Klimchitskaya, and V. M. Mostepanenko, 2002b, Phys. Rev. A 66, 032113.

Chen, F., U. Mohideen, G. L. Klimchitskaya, and V. M. Mostepanenko, 2005, Phys. Rev. A 72, 020101(R).

Chen, F., U. Mohideen, G. L. Klimchitskaya, and V. M. Mostepanenko, 2006, Phys. Rev. A 74, 022103.

Chen, F., U. Mohideen, G. L. Klimchitskaya, and V. M. Mostepanenko, 2007, Phys. Rev. Lett. 98, 068901.

Chen, F., U. Mohideen, and P. W. Milonni, 2004, in Quantum Field Theory Under the Influence of External Conditions, edited by K. A. Milton (Rinton, Princeton, NJ).

Chettiar, U. K., A. V. Kildishev, H.-K. Yuan, W.-S. Cai, S.-M. Xiao, V. P. Drachev, and V. M. Shalaev, 2007, Opt. Lett. 32, 1671.

Chiu, H.-C., C.-C. Chang, R. Castillo-Garza, F. Chen, and U. Mohideen, 2008, J. Phys. A: Math. Theor. 41, 164022.

Chumak, A. A., P. W. Milonni, and G. P. Berman, 2004, Phys. Rev. B 70, 085407.

Cochran, W. G., 1954, Biometrics 10, 101.

Contreras-Reyes, A. M., and W. L. Mochán, 2005, Phys. Rev. A 72, 034102 .

Côté, R., B. Segev, and M. G. Raizen, 1998, Phys. Rev. A 58, 3999.

Dalvit, D. A. R., and S. K. Lamoreaux, 2008, Phys. Rev. Lett. 101, 163203.

Dalvit, D. A. R., P. A. Maia Neto, A. Lambrecht, and S. Reynaud, 2008, Phys. Rev. Lett. 100, 040405.

Decca, R. S., E. Fischbach, G. L. Klimchitskaya, D. E. Krause, D. López, U. Mohideen, and V. M. Mostepanenko, 2009, Phys. Rev. A 79, 026101.

Decca, R. S., E. Fischbach, G. L. Klimchitskaya, D. E. Krause, D. López, and V. M. Mostepanenko, 2003, Phys. Rev. D 68, 116003.

Decca, R. S., D. López, H. B. Chan, E. Fischbach, D. E. Krause, and C. R. Jamell, 2005, Phys. Rev. Lett. 94, 240401.

Decca, R. S., D. López, E. Fischbach, G. L. Klimchitskaya, D. E. Krause, and V. M. Mostepanenko, 2004, J. Low Temp. Phys. 135, 63.

Decca, R. S., D. López, E. Fischbach, G. L. Klimchitskaya, D. E. Krause, and V. M. Mostepanenko, 2005, Ann. Phys. (N.Y.) 318, 37.

Decca, R. S., D. López, E. Fischbach, G. L. Klimchitskaya, D. E. Krause, and V. M. Mostepanenko, 2007a, Phys. Rev. D 75, 077101.

Decca, R. S., D. López, E. Fischbach, G. L. Klimchitskaya, D. E. Krause, and V. M. Mostepanenko, 2007b, Eur. Phys. J. C 51, 963.

Decca, R. S., D. López, E. Fischbach, and D. E. Krause, 2003, Phys. Rev. Lett. 91, 050402.

Derjaguin, B., 1934, Kolloid-Z. 69, 155.

Derjaguin, B., N. V. Churaev, and Ya. I. Rabinovich, 1987, Adv. Colloid Interface Sci. 28, 197.

Doyle, J. M., J. C. Sandberg, I. A. Yu, C. L. Cesar, D. Kleppner, and T. J. Greytak, 1991, Phys. Rev. Lett. 67, 603.

Druzhinina, V., and M. DeKieviet, 2003, Phys. Rev. Lett. 91,
193202.

Duraffourg, L., and P. Andreucci, 2006, Phys. Lett. A 359, 406. Dzyaloshinskii, I. E., E. M. Lifshitz, and L. P. Pitaevskii, 1961, Usp. Fiz. Nauk 73, 381 [ Adv. Phys. 38, 165 (1961)]. Eberlein, C., and R. Zietal, 2007, Phys. Rev. A 75, 032516.

Ederth, T., 2000, Phys. Rev. A 62, 062104.

Emig, T., 2007, Phys. Rev. Lett. 98, 160801.

Emig, T., 2008, J. Stat. Mech.: Theory Exp. (2008), P04007.

Emig, T., and R. Büscher, 2004, Nucl. Phys. B 696, 468.

Emig, T., N. Graham, R. L. Jaffe, and M. Kardar, 2007, Phys.

Rev. Lett. 99, 170403.

Emig, T., N. Graham, R. L. Jaffe, and M. Kardar, 2008, Phys. Rev. D 77, 025005.

Emig, T., A. Hanke, R. Golestanian, and M. Kardar, 2001, Phys. Rev. Lett. 87, 260402.

Emig, T., A. Hanke, R. Golestanian, and M. Kardar, 2003, Phys. Rev. A 67, 022114.

Emig, T., R. L. Jaffe, M. Kardar, and A. Scardicchio, 2006, Phys. Rev. Lett. 96, 080403.

Esquivel, R., and V. B. Svetovoy, 2004, Phys. Rev. A 69, 062102.

Esquivel, R., C. Villarreal, and W. L. Mochán, 2003, Phys. Rev. A 68, 052103; 71, 029904(E) (2005).

Feinberg, J., A. Mann, and M. Revzen, 2001, Ann. Phys. (N.Y.) 288, 103.

Fermani, R., S. Scheel, and P. L. Knight, 2007, Phys. Rev. A 75, 062905.

Fischbach, E., D. E. Krause, V. M. Mostepanenko, and M. Novello, 2001, Phys. Rev. D 64, 075010.

Friedrich, H., G. Jacoby, and C. G. Meister, 2002, Phys. Rev. A 65, 032902.

Friedrich, H., and J. Trost, 2004, Phys. Rep. 397, 359.

Genet, C., A. Lambrecht, P. Maia Neto, and S. Reynaud, 2003, Europhys. Lett. 62, 484.

Genet, C., A. Lambrecht, and S. Reynaud, 2000, Phys. Rev. A 62, 012110.

Genet, C., A. Lambrecht, and S. Reynaud, 2003, Phys. Rev. A 67, 043811.

Gerlach, E., 1971, Phys. Rev. B 4, 393.

Geyer, B., G. L. Klimchitskaya, U. Mohideen, and V. M. Mostepanenko, 2008, Phys. Rev. A 77, 036102.

Geyer, B., G. L. Klimchitskaya, and V. M. Mostepanenko, 2001, Int. J. Mod. Phys. A 16, 3291.

Geyer, B., G. L. Klimchitskaya, and V. M. Mostepanenko, 2003, Phys. Rev. A 67, 062102.

Geyer, B., G. L. Klimchitskaya, and V. M. Mostepanenko, 2004, Phys. Rev. A 70, 016102.

Geyer, B., G. L. Klimchitskaya, and V. M. Mostepanenko, 2005a, Phys. Rev. A 72, 022111.

Geyer, B., G. L. Klimchitskaya, and V. M. Mostepanenko, 2005b, Phys. Rev. D 72, 085009.

Geyer, B., G. L. Klimchitskaya, and V. M. Mostepanenko, 2006, Int. J. Mod. Phys. A 21, 5007.

Geyer, B., G. L. Klimchitskaya, and V. M. Mostepanenko, 2007, J. Phys. A: Math. Theor. 40, 13485.

Geyer, B., G. L. Klimchitskaya, and V. M. Mostepanenko, 2008, Ann. Phys. (N.Y.) 323, 291.

Gies, H., and K. Klingmüller, 2006a, Phys. Rev. Lett. 96, 220401.

Gies, H., and K. Klingmüller, 2006b, Phys. Rev. D 74, 045002. Gies, H., and K. Klingmüller, 2006c, Phys. Rev. Lett. 97, 220405. 
Ginzburg, V. L., 1985, Physics and Astrophysics (Pergamon, Oxford).

Golestanian, R., and M. Kardar, 1997, Phys. Rev. Lett. 78, 3421.

Golestanian, R., and M. Kardar, 1998, Phys. Rev. A 58, 1713.

Greenaway, D. L., G. Harbeke, F. Bassani, and E. Tosatti, 1969, Phys. Rev. 178, 1340.

Harber, D. M., J. M. McGuirk, J. M. Obrecht, and E. A. Cornell, 2003, J. Low Temp. Phys. 133, 229.

Harber, D. M., J. M. Obrecht, J. M. McGuirk, and E. A. Cornell, 2005, Phys. Rev. A 72, 033610.

Hargreaves, C. M., 1965, Proc. K. Ned. Akad. Wet., Ser. B: Phys. Sci. 68, 231.

Harris, B. W., F. Chen, and U. Mohideen, 2000, Phys. Rev. A 62, 052109.

Henkel, C., and K. Joulain, 2005, Europhys. Lett. 72, 929.

Henkel, C., K. Joulain, J.-P. Mulet, and J.-J. Greffet, 2004, Phys. Rev. A 69, 023808.

Hough, D. B., and L. R. White, 1980, Adv. Colloid Interface Sci. 14, 3 .

Høye, J. S., I. Brevik, J. B. Aarseth, and K. A. Milton, 2003, Phys. Rev. E 67, 056116.

Høye, J. S., I. Brevik, J. B. Aarseth, and K. A. Milton, 2006, J. Phys. A 39, 6031.

Høye, J. S., I. Brevik, S. A. Ellingsen, and J. B. Aarseth, 2007, Phys. Rev. E 75, 051127.

Høye, J. S., I. Brevik, S. A. Ellingsen, and J. B. Aarseth, 2008, Phys. Rev. E 77, 023102.

Iannuzzi, D., I. Gelfand, M. Lisanti, and F. Capasso, 2004, in Quantum Field Theory under the Influence of External Conditions, edited by K. A. Milton (Rinton, Princeton, NJ).

Iannuzzi, D., M. Lisanti, and F. Capasso, 2004, Proc. Natl. Acad. Sci. U.S.A. 101, 4019.

Intravaia, F., and C. Henkel, 2008, J. Phys. A: Math. Theor. 41, 164018.

Intravaia, F., C. Henkel, and A. Lambrecht, 2007, Phys. Rev. A 76, 033820 .

Intravaia, F., and A. Lambrecht, 2005, Phys. Rev. Lett. 94, 110404.

Inui, N., 2003, J. Phys. Soc. Jpn. 72, 2198.

Inui, N., 2004, J. Phys. Soc. Jpn. 73, 332.

Inui, N., 2006, J. Phys. Soc. Jpn. 75, 024004.

Israelashvili, J., 1992, Intermolecular and Surface Forces (Academic, New York).

Jackson, J. D., 1999, Classical Electrodynamics (Wiley, New York).

Jaffe, R. L., 2005, Phys. Rev. D 72, 021301.

Jourdan, G., A. Lambrecht, F. Comin, and J. Chevrier, 2009, EPL 85, 31001.

Jurisch, A., and H. Friedrich, 2004, Phys. Rev. A 70, 032711.

Jurisch, A., and H. Friedrich, 2006, Phys. Lett. A 349, 230.

Kardar, M., and R. Golestanian, 1999, Rev. Mod. Phys. 71, 1233.

Kats, E. I., 1977, Zh. Eksp. Teor. Fiz. 73, 212 [Sov. Phys. JETP 46, 109 (1977)].

Kenneth, O., and I. Klich, 2006, Phys. Rev. Lett. 97, 160401.

Kenneth, O., and I. Klich, 2008, Phys. Rev. B 78, 014103.

Kenneth, O., I. Klich, A. Mann, and M. Revzen, 2002, Phys. Rev. Lett. 89, 033001.

Kim, W. J., M. Brown-Hayes, D. A. R. Dalvit, J. H. Brownell, and R. Onofrio, 2008, Phys. Rev. A 78, 020101(R).

Kittel, C., 1996, Introduction to Solid State Physics (Wiley, New York).
Klimchitskaya, G. L., 2009, J. Phys.: Conf. Ser. 161, 012002. Klimchitskaya, G. L., E. V. Blagov, and V. M. Mostepanenko, 2006, J. Phys. A 39, 6481.

Klimchitskaya, G. L., E. V. Blagov, and V. M. Mostepanenko, 2008, J. Phys. A: Math. Theor. 41, 164012.

Klimchitskaya, G. L., F. Chen, R. S. Decca, E. Fischbach, D. E. Krause, D. López, U. Mohideen, and V. M. Mostepanenko, 2006, J. Phys. A 39, 6485.

Klimchitskaya, G. L., R. S. Decca, E. Fischbach, D. E. Krause, D. López, and V. M. Mostepanenko, 2005, Int. J. Mod. Phys. A 20, 2205.

Klimchitskaya, G. L., and B. Geyer, 2008, J. Phys. A: Math. Theor. 41, 164032.

Klimchitskaya, G. L., B. Geyer, and V. M. Mostepanenko, 2006, J. Phys. A 39, 6495.

Klimchitskaya, G. L., U. Mohideen, and V. M. Mostepanenko, 2000, Phys. Rev. A 61, 062107.

Klimchitskaya, G. L., U. Mohideen, and V. M. Mostepanenko, 2007a, J. Phys. A: Math. Theor. 40, F339.

Klimchitskaya, G. L., U. Mohideen, and V. M. Mostepanenko, 2007b, J. Phys. A: Math. Theor. 40, F841.

Klimchitskaya, G. L., U. Mohideen, and V. M. Mostepanenko, 2008, J. Phys. A: Math. Theor. 41, 432001.

Klimchitskaya, G. L., and V. M. Mostepanenko, 2001, Phys. Rev. A 63, 062118.

Klimchitskaya, G. L., and V. M. Mostepanenko, 2006, Contemp. Phys. 47, 131.

Klimchitskaya, G. L., and V. M. Mostepanenko, 2007, Phys. Rev. B 75, 036101.

Klimchitskaya, G. L., and V. M. Mostepanenko, 2008a, Phys. Rev. E 77, 023101.

Klimchitskaya, G. L., and V. M. Mostepanenko, 2008b, J. Phys. A: Math. Theor. 41, 312002(F).

Klimchitskaya, G. L., and Yu. V. Pavlov, 1996, Int. J. Mod. Phys. A 11, 3723.

Klimchitskaya, G. L., A. Roy, U. Mohideen, and V. M. Mostepanenko, 1999, Phys. Rev. A 60, 3487.

Klimchitskaya, G. L., S. I. Zanette, and A. O. Caride, 2000, Phys. Rev. A 63, 014101.

Kondepugi, D., and I. Prigogine, 1998, Modern Thermodynamics (Wiley, New York).

Kouznetsov, D., and H. Oberst, 2005, Phys. Rev. A 72, 013617.

Krause, D. E., R. S. Decca, D. López, and E. Fischbach, 2007, Phys. Rev. Lett. 98, 050403.

Krech, M., 1994, The Casimir Effect in Critical Systems (World Scientific, Singapore).

Lambrecht, A., P. A. Maia Neto, and S. Reynaud, 2006, New J. Phys. 8, 243.

Lambrecht, A., and V. N. Marachevsky, 2008, Phys. Rev. Lett. 101, 160403.

Lambrecht, A., V. V. Nesvizhevsky, R. Onofrio, and S. Reynaud, 2005, Class. Quantum Grav. 22, 5397.

Lambrecht, A., I. Pirozhenko, L. Duraffourg, and P. Andreucci, 2007, EPL 77, 44006; 81, 19901(E) (2008).

Lambrecht, A., and S. Reynaud, 2000, Eur. Phys. J. D 8, 309.

Lamoreaux, S. K., 1997, Phys. Rev. Lett. 78, 5.

Lamoreaux, S. K., 1998, Phys. Rev. Lett. 81, 5475(E).

Lamoreaux, S. K., 1999, Phys. Rev. A 59, R3149.

Lamoreaux, S. K., 2005, Rep. Prog. Phys. 68, 201.

Lamoreaux, S. K., and W. T. Buttler, 2005, Phys. Rev. E 71, 036109.

Landau, L. D., and E. M. Lifshitz, 1980, Statistical Physics (Pergamon, Oxford), Pt. I. 
Landau, L. D., E. M. Lifshitz, and L. P. Pitaevskii, 1984, Electrodynamics of Continuous Media (Pergamon, Oxford).

Lehmann, E. L., and J. P. Romano, 2005, Testing Statistical Hypotheses (Springer, New York).

Lennard-Jones, J. E., and A. F. Devonshire, 1936, Proc. R. Soc. London 156, 6 .

Leonhardt, U., and T. G. Philbin, 2007, New J. Phys. 9, 254.

Lifshitz, E. M., 1956, Zh. Eksp. Teor. Fiz. 29, 94 [ Sov. Phys. JETP 2, 73 (1956)].

Lifshitz, E. M., and L. P. Pitaevskii, 1980, Statistical Physics (Pergamon, Oxford), Pt. II.

Lifshitz, E. M., and L. P. Pitaevskii, 1981, Physical Kinetics (Pergamon, Oxford).

Lifshitz, I. M., M. Ya. Azbel', and M. I. Kaganov, 1973, Electron Theory of Metals (Consultants Bureau, New York).

Lisanti, M., D. Iannuzzi, and F. Capasso, 2005, Proc. Natl. Acad. Sci. U.S.A. 102, 11989.

London, F., 1930, Z. Phys. 63, 245.

Long, J. C., H. W. Chan, and J. C. Price, 1999, Nucl. Phys. B 539, 23.

Lukosz, W., 1971, Physica (Amsterdam) 56, 109.

Madroñero, J., and H. Friedrich, 2007, Phys. Rev. A 75, 022902.

Mahanty, J., and B. W. Ninham, 1976, Dispersion Forces (Academic, New York).

Maia Neto, P. A., A. Lambrecht, and S. Reynaud, 2005, Phys. Rev. A 72, 012115.

Maia Neto, P. A., A. Lambrecht, and S. Reynaud, 2008, Phys. Rev. A 78, 012115.

Maradudin, A. A., and P. Mazur, 1980, Phys. Rev. B 22, 1677.

Mazur, P., and A. A. Maradudin, 1981, Phys. Rev. B 23, 695.

McGuirk, J. M., D. M. Harber, J. M. Obrecht, and E. A. Cornell, 2004, Phys. Rev. A 69, 062905.

Mehra, J., 1967, Physica (Amsterdam) 37, 145.

Meurk, A., P. F. Luckham, and L. Bergström, 1997, Langmuir 13, 3896

Milonni, P. W., 1994, The Quantum Vacuum. An Introduction to Quantum Electrodynamics (Academic, San Diego).

Milonni, P. W., 2007, Phys. Scr. 76, C167.

Milton, K. A., 2001, The Casimir Effect: Physical Manifestation of Zero-Point Energy (World Scientific, Singapore).

Milton, K. A., 2004, J. Phys. A 37, R209.

Mohideen, U., and A. Roy, 1998, Phys. Rev. Lett. 81, 4549.

Mostepanenko, V. M., J. F. Babb, A. O. Caride, G. L. Klimchitskaya, and S. I. Zanette, 2006, J. Phys. A 39, 6583.

Mostepanenko, V. M., V. B. Bezerra, R. S. Decca, E. Fischbach, B. Geyer, G. L. Klimchitskaya, D. E. Krause, D. López, and C. Romero, 2006, J. Phys. A 39, 6589.

Mostepanenko, V. M., R. S. Decca, E. Fischbach, G. Geyer, G. L. Klimchitskaya, D. E. Krause, D. López, and U. Mohideen, 2009, Int. J. Mod. Phys. A 24, 1721.

Mostepanenko, V. M., R. S. Decca, E. Fischbach, G. L. Klimchitskaya, D. E. Krause, and D. López, 2008, J. Phys. A: Math. Theor. 41, 164054.

Mostepanenko, V. M., and B. Geyer, 2008, J. Phys. A: Math. Theor. 41, 164014.

Mostepanenko, V. M., and M. Novello, 2001, Phys. Rev. D 63, 115003.

Mostepanenko, V. M., and N. N. Trunov, 1985, Yad. Fiz. 42, 1297 [ Sov. J. Nucl. Phys. 42, 818 (1985)].

Mostepanenko, V. M., and N. N. Trunov, 1988, Usp. Fiz. Nauk 156, 385 [ Sov. Phys. Usp. 31, 965 (1988)].

Mostepanenko, V. M., and N. N. Trunov, 1997, The Casimir
Effect and its Applications (Clarendon, Oxford).

Mott, N. F., 1990, Metal-Insulator Transitions, 2nd ed. (Taylor \& Francis, London).

Munday, J. N., and F. Capasso, 2007, Phys. Rev. A 75, 060102(R).

Munday, J. N., and F. Capasso, 2008, Phys. Rev. A 77, 036103. Munday, J. N., F. Capasso, and V. A. Parsegian, 2009, Nature (London) 457, 170.

Munday, J. N., F. Capasso, V. A. Parsegian, and S. M. Bezrukov, 2008, Phys. Rev. A 78, 032109.

Munday, J. N., D. Iannuzzi, Yu. Barash, and F. Capasso, 2005, Phys. Rev. A 71, 042102.

Nagai, M., and M. Kuwata-Gonokami, 2002, J. Phys. Soc. Jpn. 71, 2276.

Nayak, V. U., D. E. Edwards, and N. Masuhara, 1983, Phys. Rev. Lett. 50, 990.

Ninham, B. W., V. A. Parsegian, and J. H. Weiss, 1970, J. Stat. Phys. 2, 323.

Oberst, H., D. Kouznetsov, K. Shimizu, J.-I. Fujita, and F. Shimizu, 2005, Phys. Rev. Lett. 94, 013203.

Oberst, H., Y. Taashiro, K. Shimizu, and F. Shimizu, 2005, Phys. Rev. A 71, 052901.

Obrecht, J. M., R. J. Wild, M. Antezza, L. P. Pitaevskii, S. Stringari, and E. A. Cornell, 2007, Phys. Rev. Lett. 98, 063201.

Palik, E. D., 1985, Ed., Handbook of Optical Constants of Solids (Academic, New York).

Parsegian, V. A., 2005, van der Waals Forces: A Handbook for Biologists, Chemists, Engineers, and Physicists (Cambridge University Press, Cambridge).

Parsegian, V. A., and G. H. Weiss, 1972, Adv. Colloid Interface Sci. 40, 35.

Pasquini, T. A., M. Saba, G.-B. Jo, Y. Shin, W. Ketterle, and D. E. Pritchard, 2006, Phys. Rev. Lett. 97, 093201.

Pasquini, T. A., Y. Shin, C. Sanner, M. Saba, A. Schirotzek, D. E. Pritchard, and W. Ketterle, 2004, Phys. Rev. Lett. 93, 223201.

Pendry, J. B., A. J. Holden, D. J. Robbins, and W. J. Stewart, 1999, IEEE Trans. Microwave Theory Tech. 47, 2075

Petrov, V., M. Petrov, V. Bryksin, J. Petter, and T. Tschudi, 2006, Opt. Lett. 31, 3167.

Philbin, T. G., and U. Leonhardt, 2008, Phys. Rev. A 78, 042107.

Pirozhenko, I., and A. Lambrecht, 2008a, Phys. Rev. A 77, 013811.

Pirozhenko, I., and A. Lambrecht, 2008b, J. Phys. A: Math. Theor. 41, 164015.

Pirozhenko, I., A. Lambrecht, and V. B. Svetovoy, 2006, New J. Phys. 8, 238.

Pitaevskii, L. P., 2008, Phys. Rev. Lett. 101, 163202.

Plunien, G., B. Müller, and W. Greiner, 1986, Phys. Rep. 134, 87.

Raabe, C., and D.-G. Welsch, 2006, Phys. Rev. A 73, 063822.

Rabinovich, S. G., 2000, Measurement Errors and Uncertainties. Theory and Practice (Springer, New York).

Rabinovich, Ya. I., and N. V. Churaev, 1989, Kolloidn. Zh. 51, 83 [Colloid J. USSR 51, 65 (1989)].

Rodrigues, R. B., P. A. Maia Neto, A. Lambrecht, and S. Reynaud, 2006a, Phys. Rev. Lett. 96, 100402.

Rodrigues, R. B., P. A. Maia Neto, A. Lambrecht, and S. Reynaud, 2006b, Europhys. Lett. 76, 822.

Rodrigues, R. B., P. A. Maia Neto, A. Lambrecht, and S. Reynaud, 2007, Phys. Rev. A 75, 062108. 
Rodriguez, A., M. Ibanescu, D. Iannuzzi, J. D. Joannopoulos, and S. G. Johnson, 2007, Phys. Rev. A 76, 032106.

Rosa, F. S. S., D. A. R. Dalvit, and P. W. Milonni, 2008, Phys. Rev. Lett. 100, 183602.

Roy, A., C.-Y. Lin, and U. Mohideen, 1999, Phys. Rev. D 60, 111101(R).

Roy, A., and U. Mohideen, 1999, Phys. Rev. Lett. 82, 4380.

Rumer, Yu. B., and M. S. Ryvkin, 1980, Thermodynamics, Statistical Physics, and Kinetics (Mir, Moscow).

Sabbah, A. J., and D. M. Riffe, 2002, Phys. Rev. B 66, 165217. Saharian, A. A., 2006, Phys. Rev. D 73, 064019.

Saito, R., G. Dresselhaus, and M. S. Dresselhaus, 1998, Physical Properties of Carbon Nanotubes (Imperial College Press, London).

Scardicchio, A., and R. L. Jaffe, 2006, Nucl. Phys. B 743, 249.

Schram, K., 1973, Phys. Lett. 43A, 282.

Schwinger, J., L. L. DeRaad, and K. A. Milton, 1978, Ann. Phys. (N.Y.) 115, 1.

Sernelius, B. E., 2005, Phys. Rev. B 71, 235114.

Shackelford, J. F., and W. Alexander, 2001, Eds., Material Science and Engineering Handbook (CRC, Boca Raton).

Shalaev, V. M., 2007, Nature Photon. 1, 41.

Shih, A., and V. A. Parsegian, 1975, Phys. Rev. A 12, 835.

Shimizu, F., 2001, Phys. Rev. Lett. 86, 987.

Shklovskii, B. I., and A. L. Efros, 1984, Electronic Properties of Doped Semiconductors, Solid State Series Vol. 45 (Springer, Berlin).

Smythe, W. R., 1950, Electrostatics and Electrodynamics (McGraw-Hill, New York).

Soltani, M., M. Chaker, E. Haddad, R. V. Kruzelecky, and D. Nikanpour, 2004, J. Vac. Sci. Technol. A 22, 859.

Sotelo, J., J. Ederth, and G. Niklasson, 2003, Phys. Rev. B 67, 195106.

Spagnolo, S., D. A. R. Dalvit, and P. W. Milonni, 2007, Phys. Rev. A 75, 052117.

Sparnaay, M. J., 1958, Physica (Amsterdam) 24, 751.

Sparnaay, M. J., 1989, in Physics in the Making, edited by A. Sarlemijn and M. J. Sparnaay (North-Holland, Amsterdam). Speake, C. C., and C. Trenkel, 2003, Phys. Rev. Lett. 90, 160403.
Suh, J. Y., R. Lopez, L. C. Feldman, and R. F. Haglund, 2004, J. Appl. Phys. 96, 1209.

Sukenik, C. I., M. G. Boshier, D. Cho, V. Sandoghbar, and E. A. Hinds, 1993, Phys. Rev. Lett. 70, 560.

Svetovoy, V. B., 2008, Phys. Rev. Lett. 101, 163603.

Svetovoy, V. B., and R. Esquivel, 2005, Phys. Rev. E 72, 036113.

Svetovoy, V. B., and M. V. Lokhanin, 2003, Phys. Rev. A 67, 022113.

Svetovoy, V. B., P. J. van Zwol, G. Palasantzas, and J. Th. M. De Hosson, 2008, Phys. Rev. B 77, 035439.

Tomaš, M. S., 2002, Phys. Rev. A 66, 052103.

Tomaš, M. S., 2005, Phys. Lett. A 342, 381.

Torgerson, J. R., and S. K. Lamoreaux, 2004, Phys. Rev. E 70, 047102.

van Blockland, P. H. G. M., and J. T. G. Overbeek, 1978, J. Chem. Soc., Faraday Trans. 1 74, 2637.

Van Kampen, N. G., B. R. A. Nijboer, and K. Schram, 1968, Phys. Lett. 26A, 307.

van Zwol, P. J., G. Palasantzas, and J. Th. M. De Hosson, 2007, Appl. Phys. Lett. 91, 144108.

van Zwol, P. J., G. Palasantzas, M. van de Schootbrugge, and J. Th. M. De Hosson, 2008, Appl. Phys. Lett. 92, 054101.

Verleur, H. W., A. S. Barker, and C. N. Berglund, 1968, Phys. Rev. 172, 788.

Visser, J., 1981, Adv. Colloid Interface Sci. 15, 157.

Vogel, T., G. Dodel, E. Holzhauer, H. Salzmann, and A. Theurer, 1992, Appl. Opt. 31, 329.

Volokitin, A. I., and B. N. J. Persson, 2007, Rev. Mod. Phys. 79, 1291.

Voronin, A. Yu., and P. Froelich, 2005, J. Phys. B 38, L301.

Voronin, A. Yu., P. Froelich, and B. Zygelman, 2005, Phys. Rev. A 72, 062903.

Yu, I. A., J. M. Doyle, J. C. Sandberg, C. L. Cesar, D. Kleppner, and T. J. Greytak, 1993, Phys. Rev. Lett. 71, 1589.

Zhou, F., and L. Spruch, 1995, Phys. Rev. A 52, 297.

Zurita-Sánchez, J. R., J.-J. Greffet, and L. Novotny, 2004, Phys. Rev. A 69, 022902.

Zylbersztejn, A., and N. F. Mott, 1975, Phys. Rev. B 11, 4383. 